\title{
Phanerogamic flora and vegetation of Itacolomi State Park, Minas Gerais, Brazil
}

\author{
Maria Cristina T. Braga Messias ${ }^{1 *}$, Hildeberto Caldas de Sousa ${ }^{1}$, Viviane Renata Scalon ${ }^{1}$, Miriam B. Roschel ${ }^{1}$, \\ Elisa Silva Cândido ${ }^{1,2}$ \& Maria Augusta G. Fujaco ${ }^{3}$ \\ ${ }^{1}$ Departamento de Biodiversidade, Evolução e Meio Ambiente, Programa de Pós-Graduação em Ecologia de Biomas \\ Tropicais, ICEB, Universidade Federal de Ouro Preto, Ouro Preto, MG, Brazil \\ ${ }^{2}$ Programa de Pós Graduação em Biologia Vegetal, Instituto de Biologia, Universidade Estadual de Campinas, \\ Campinas, SP, Brazil \\ ${ }^{3}$ Departamento de Geologia, Universidade Federal de Ouro Preto, Ouro Preto, MG, Brazil \\ *Corresponding author: Maria Cristina T. Braga Messias, e-mail: cristinabotanica@gmail.com
}

\begin{abstract}
MESSIAS, M.C.T.B., SOUSA, H.C., SCALON, V., ROSCHEL, M. B., CANDIDO, E.S., FUJACO, M.A.G. Phanerogamic flora and vegetation of Itacolomi State Park, Minas Gerais, Brazil. Biota Neotropica. 17(1): e20160236. http:// dx.doi.org/10.1590/1676-0611-BN-2016-0236
\end{abstract}

\begin{abstract}
Located in the municipalities of Ouro Preto and Mariana, in the Quadrilátero Ferrifero (QF) of Minas Gerais, the Itacolomi State Park (ISP) shelters more than 7,000 ha of remnants of Atlantic Forest and campos rupestres. The QF region has high biodiversity and is being highly impacted, mainly by mining activities. Aiming to organize the available floristic information and to support related research, this study presents the list of phanerogamic species of the ISP and the major vegetation types. This survey was carried out from October/1992 to July/2006, by monthly field trips. Besides authors' personal collections, other records were assembled from herbarium databank. A total of 1623 taxons belonging to 122 families were listed. The families with higher species richness were Asteraceae, Fabaceae, Melastomataceae, Poaceae and Orchidaceae. The vegetation is represented by campos rupestres (51\% of the total area), followed by montane forests (40\%) and anthropogenic disturbed areas (9\%). The greatest species richness occurs in campos rupestres. Several species are threatened and/or endemic. The knowledge of phanerogamic flora of ISP can help the Management Plan of this Unit of Conservation. It is also a contribution for future-related studies of the flora of ISP, Minas Gerais and Brazil.
\end{abstract}

Keywords: Brazilian flora, campos rupestres, Iron Quadrangle flora, montane forests, rock outcrop flora, Neotropics.

\section{Flora Fanerogâmica e Vegetação do Parque Estadual do Itacolomi, Minas Gerais, Brasil}

Resumo: O Parque Estadual do Itacolomi (PEIT) localiza-se nos municípios de Ouro Preto e Mariana, no Quadrilátero Ferrífero de Minas Gerais e abriga mais de 7.000 ha de remanescentes de mata atlântica e campos rupestres. A região do QF possui alta biodiversidade vegetal e está sobre alto impacto, principalmente pela mineração. O objetivo deste estudo foi apresentar a lista das espécies fanerogâmicas e os principais tipos vegetacionais desse Parque, no intuito de organizar a diversidade florística existente, além de servir como suporte para pesquisas correlatas subsequentes. O levantamento florístico foi realizado de outubro/1992 a julho/2006 por excursões mensais ao campo. Foram também incluídas na listagem registros ocorrentes em banco de dados de herbários. Foram identificados 1623 táxons, pertencentes à 122 famílias. As famílias mais representativas foram Asteraceae, Fabaceae, Melastomataceae, Poaceae e Orchidaceae. A vegetação do PEIT é constituída por campos rupestres ( $51 \%$ da área do parque), seguido pelas florestas montanas (40\%) e por formações antrópicas (9\%). A maior riqueza de espécies ocorre nos campos rupestres. Diversas espécies figuram em listas de espécies ameaçadas de extinção e outras são endêmicas. O conhecimento da flora fanerogâmica do Parque Estadual do Itacolomi pode subsidiar o Plano de Manejo dessa Unidade de Conservação e contribuir para futuros trabalhos correlatos nesta área, além de suprir conhecimentos para a flora de Minas Gerais e do Brasil.

Palavras-chave: campos rupestres, flora do Quadrilátero Ferrifero, flora do Brasil, florestas montanas, Neotropicos.

\section{Introduction}

Minas Gerais is the Brazilian State with the highest richness and endemism of Angiosperm species and harbors important phytogeographical domains (BFG 2015). Among them, the Atlantic Forest and the Cerrado are the largest, richest and more threatened ones (Fundação SOS Mata Atlântica 1998, Coutinho 2006). Atlantic Forest is one of the most biologically rich and diverse domain in the world, with around 20,000 plant species, representing $8 \%$ of the Earth's flora (Fundação SOS Mata Atlântica 1998). In spite of the Atlantic Forest being considered 
a biodiversity hotspot (Myers et al. 2000), it was reduced to less than $12 \%$ of its original size, with most of the remaining areas restricted to Conservation Units (Ribeiro et al. 2009).

With regard to Brazilian vegetation, the campos rupestres stand out due to the peculiar and rich biodiversity. They occur from an altitude of $900 \mathrm{~m}$ above sea level and upwards, mainly along the Espinhaço Range, in the States of Minas Gerais and Bahia, and with disjunct parts of the same chain, between latitudinal limits of $21^{\circ} 10^{\prime}$ and $10^{\circ} \mathrm{S}$ (Giulietti \& Pirani 1988). Phytogeographically, the campos rupestres are classified into the Cerrado domain (Coutinho 2006, Batalha 2011), a kind of savanna, where plants grow in a wide variety of substrates including rock outcrops of quartzite, sandstone or ironstone, along with sandy, stony and waterlogged grasslands (Silveira et al. 2016). Campos rupestres also harbor several endemic and threatened species from different groups of organisms (Echternacht et al. 2011, BGF 2015, Silveira et al. 2016). Among the 538 threatened plant species of the Minas Gerais State, 358 (66.5 percent) occur in this kind of physiognomy (Costa et al. 1998). Among the Brazilian vegetation types, the campos rupestres have the highest percentage of endemism (1,951 endemic out of 4,928 species) (BGF 2015)

The Quadrilátero Ferrifero (Iron Quadrangle) Region (QF) covers an area of $7,000 \mathrm{~km}^{2}$, in the central portion of the Minas Gerais State (Dorr 1969). This region is considered to be of extreme biological importance (Costa et al. 1998). The QF landscape is a mosaic under the influence of two Brazilian hotspots, Cerrado and Atlantic Forest, giving evidence of the ecotonal character of the region (Echternacht et al. 2011). The Atlantic Forest in the QF Region is mainly represented by a seasonal semideciduous type (Morellato \& Haddad 2000) and the Cerrado occurs in different phytophysiognomies, including the campos rupestres (Silveira et al. 2016). The campos rupestres in the Quadrilátero Ferrifero, occur frequently in areas with quartzite rocks, interspersed mainly with banded iron formation (BIF's), also known as cangas (Messias et al. 2011, 2012, 2013). Cangas are one of the most diverse, threatened and least studied ecosystems of southeast Brazil (Jacobi et al. 2007). In spite of the great biodiversity, mining, as well as metallurgical industries, urban expansion, among other activities, have provoked a high environmental impact in the Quadrilátero Ferrifero Region (Jacobi et al. 2007).

Itacolomi State Park is one of the few full protection conservation units in this region (IEF 2016) and shelters more than 7,000 ha of remnants of Atlantic Forest and campos rupestres. Most of the campos rupestres are quartzitic, but ISP is one of the three full protection parks of Minas Gerais also harboring ferruginous campos rupestres. Thus, this conservation unit in this region plays an important role in protecting biodiversity.

Little is still known about Itacolomi State Park flora, whose domain is an ecotone region between the Atlantic Forest and the Cerrado. There were found only a preliminary checklist of campos rupestres (Peron 1989), specific family studies about Orchidaceae (Alves 1990, Batista et al. 2004), Leguminosae (Dutra et al. 2005, 2008a, 2008b, 2009, 2014, Lima et al. 2007, 2010), Bromeliaceae (Coser et al. 2010), Myrtaceae (Bünger et al. 2012) and Asteraceae (Barnadesieae e Mutiseae) (Almeida et al. 2014). And also, few floristic studies have been recently published on small patches of campos rupestres (Gastauer et al. 2012) and forests (Pedreira \& Sousa 2011) of this conservation unit. The aims of this study are to present a brief description of the vegetation of Itacolomi State Park (ISP) and to present a checklist of the phanerogamic flora.

\section{Material and Methods}

This survey was carried out in Itacolomi State Park (ISP), situated in the municipalities of Ouro Preto (World Patrimony by Unesco) and Mariana (Brazilian Historic Patrimony), Minas Gerais State, (4332'30" to $43^{\circ} 22^{\prime} 30^{\prime \prime} \mathrm{W}$ and $20^{\circ} 22^{\prime} 30^{\prime \prime}$ to $20^{\circ} 30^{\prime} 00^{\prime \prime}$ ). This Brazilian Conservation Unit was created in 1967 by Minas Gerais State law n 4465 (Figure 1).

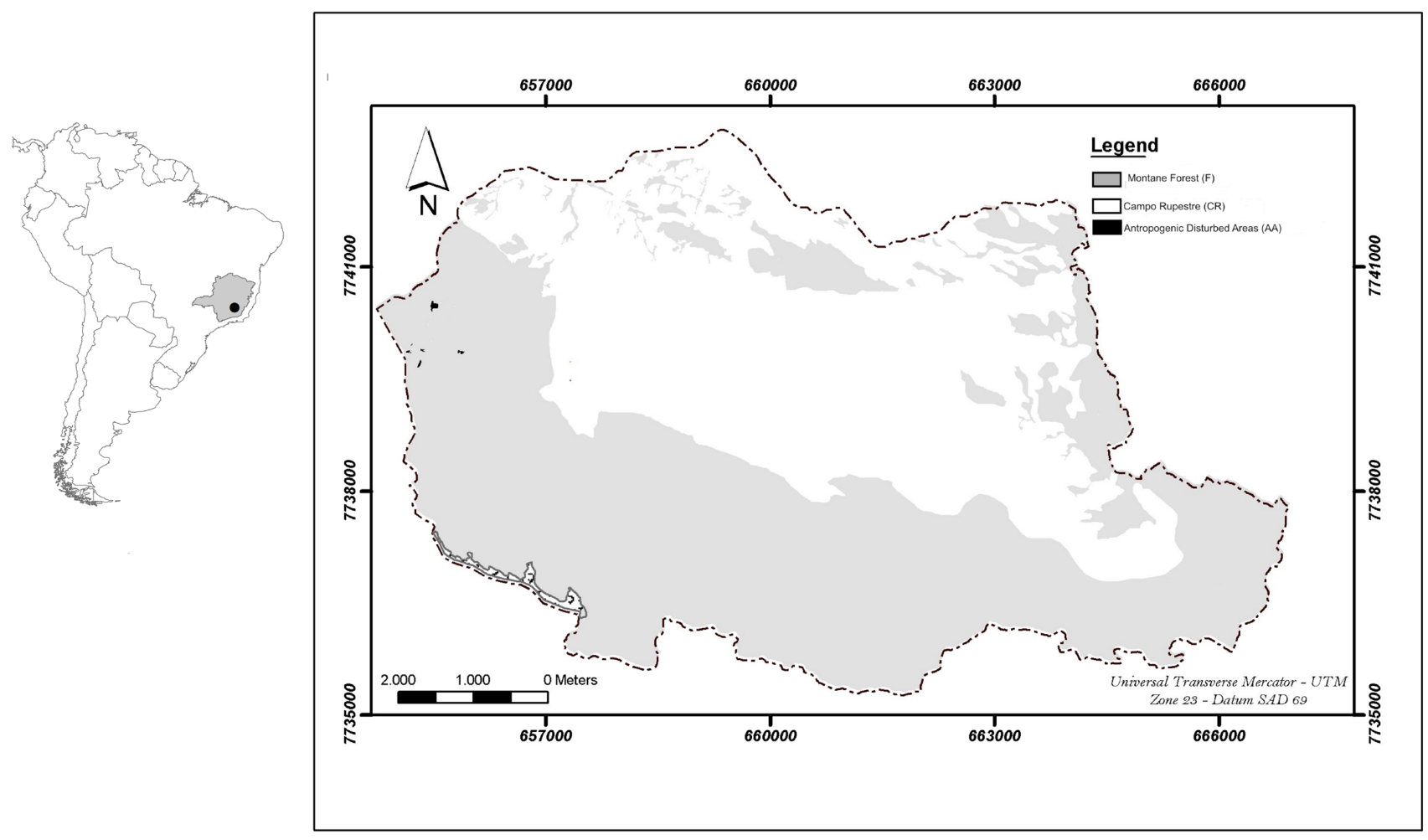

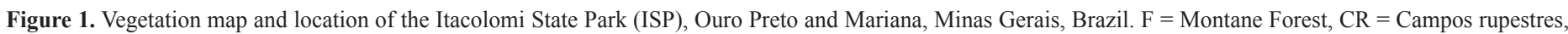
$\mathrm{AA}=$ Anthropogenic disturbed areas. 
According to Peron (1989) the vegetation at ISP is formed mainly by campos rupestres permeated by riparian or semideciduous forest fragments.

The climate, according Köppen classification is Cwb, i. e. mesotermic, with a warm and rainy season from September to April with a dry season from May to August (Álvares et al. 2013). The annual mean rainfall is $1,250 \mathrm{~mm}$ and the annual mean temperature is about $20^{\circ} \mathrm{C}$ (varying from 4 to $33^{\circ} \mathrm{C}$ ). Fog is frequent, especially during the dry season.

The relief is characterized by steep slopes where the altitudes varies from 700 to $1772 \mathrm{~m}$. The Park covers the greatest part of Itacolomi Hill, at the southern of Espinhaço Range, with around 7,500 ha (Castañeda 1993). The geology of ISP comprises clastic metasedimentary rocks from Rio das Velhas and Minas Supergroups, Sabará and Itacolomi Groups. The quartzite of Itacolomi Group occurs in the greatest proportion of the Park area. There are also intrusions of metabasic rocks and a superficial iron crusts known as canga (Castañeda 1993). According to the same author, the soils at Itacolomi vary from sandy shallow soils, mostly associated to quartzite, occurring in campos rupestres to deeper ones, mainly Latossols (Oxisols), at forest patches at lower altitudes.

This survey was carried out from October/1992 to July/2006, when monthly field trips were taken, all over the Park. The collected species were herborized and deposited in the Professor José Badini Herbarium (OUPR) at Federal University of Ouro Preto. The species identification was made by comparison with previously identified material at RB, BHCB, VIC and OUPR herbaria, by using specialized literature and with the help of specialists.

Aiming to supplement the checklist, records from SpeciesLink (CRIA 2016) and JABOT (JBRJ 2016) databank were included. Materials determined only at the family level were not considered and the ones identified at generic level were included only when deposited in OUPR and assumed they were different from the species already listed. Records included in the checklist appear cited with acronyms according to Thiers (2016).

The circumscription of families followed the Angiosperm Phylogeny Group - APG III (Chase \& Reveal 2009). The species names were checked with the database of the list of species of the Brazilian Flora 2020 Project (2016) and The Plant List (2013). Each species was described by its habit (Rizzini 1997) and habitat. The forest vegetation was classified according to Veloso et al. (1991) and the grasslands areas followed Ferri (1980), Eiten (1983) and Giulietti et al. (1987). The major vegetation types are Montane Forest (F), Campos Rupestres (CR) and Anthropogenic Disturbed Areas (AA). In order to recognize the threatened species, it was consulted the IUCN Red List (2015), as well as the Brazilian (MMA 2014) and Minas Gerais (COPAM 1997) Red Lists.

The vegetation map was made using an IKONOS image, which was provided by the State Forest Institute of Minas Gerais (IEF). The vegetation groups described were identified in the field campaigns and then designed in Geographic Information System (GIS) environment with Arcview 9.2 software (Environmental Systems Research Institute, 2007).

\section{Results and Discussion}

A total of 1614 species, one subspecies and eight varieties, belonging to 569 genera and 122 families were identified (Table 1). The species richness in ISP is comparable with other mountainous areas along the Espinhaço Range, e.g. Pico das Almas: 930 species (Stannard et al. 1995), Ibitipoca: 1080 species (Forzza et al. 2013), Grão Mogol: 1073 species (Pirani et al. 2003), Serra de São José: 1087 species (Alves \& Kolbek 2009) and Serra do Cipó: 1530 species (Giulietti et al. 1987). These results highlight how diverse is the ISP flora as well as the importance of focusing on further taxonomic studies in this park.

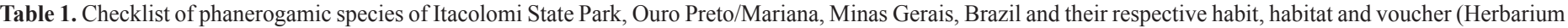

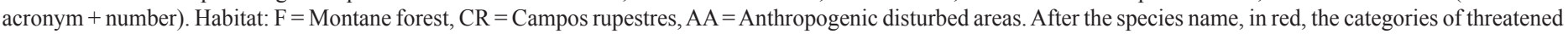

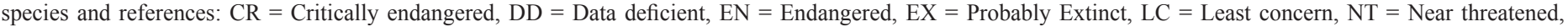
$\mathrm{VU}=$ Vulnerable, * COPAM (1997), ** MMA (2014), *** IUCN (2015).

\begin{tabular}{|c|c|c|c|}
\hline Family / Species & Habit & Habitat & Voucher \\
\hline \multicolumn{4}{|l|}{ Acanthaceae } \\
\hline Aphelandra prismatica (Vell.) Hiern & Subshrub & $\mathrm{F}$ & OUPR 9064 \\
\hline Aphelandra sp & Subshrub & $\mathrm{F}$ & OUPR 1296 \\
\hline Clistax brasiliensis Mart. & Subshrub & $\mathrm{F}$ & BHCB 41493 \\
\hline Justicia monticola (Nees) Profice & Liana & $\mathrm{CR}$ & OUPR 102 \\
\hline Justicia $\mathrm{sp} 1$ & Subshrub & $\mathrm{F}$ & OUPR 6780 \\
\hline Justicia $\mathrm{sp} 2$ & Subshrub & $\mathrm{F}$ & OUPR 6825 \\
\hline Justicia $\mathrm{sp} 3$ & Subshrub & $\mathrm{F}$ & OUPR 1403 \\
\hline Justicia $\mathrm{sp} 4$ & Subshrub & $\mathrm{F}$ & OUPR 1991 \\
\hline Justicia $\operatorname{sp} 5$ & Subshrub & $\mathrm{F}$ & OUPR 19286 \\
\hline Mendoncia puberula Mart. & Liana & CR & OUPR 045 \\
\hline Mendoncia velloziana Mart. & Liana & $\mathrm{F}$ & OUPR 1273 \\
\hline Ruellia geminiflora Kunth & Subshrub & $\mathrm{CR}$ & OUPR 061 \\
\hline Ruellia jussieuoides Schltdl. \& Cham. & Subshrub & $\mathrm{F}$ & OUPR 7352 \\
\hline Ruellia macrantha (Mart. ex Ness) Lindau & Subshrub & $\mathrm{CR}, \mathrm{F}$ & OUPR 1996 \\
\hline Ruellia neesiana (Mart. ex Ness) Lindau & Subshrub & $\mathrm{CR}$ & NY 1059877 \\
\hline Staurogyne minarum (Nees) Kuntze EN* & Subshrub & $\mathrm{F}$ & OUPR 10002 \\
\hline Staurogyne vauthieriana (Nees) Kuntze EN* & Subshrub & $\mathrm{F}$ & HRCB 42225 \\
\hline Staurogyne sp & Subshrub & $\mathrm{F}$ & OUPR 7411 \\
\hline \multicolumn{4}{|l|}{ Alismataceae } \\
\hline Echinodorus grandiflorus (Cham. \& Schltr.) Micheli & Herb & AA & NY 51987 \\
\hline \multicolumn{4}{|l|}{ Alstroemeriaceae } \\
\hline Alstroemeria foliosa Mart. ex Schult. \& Schult.f. & Herb & $\mathrm{CR}$ & OUPR 617 \\
\hline Alstroemeria haemantha Ruiz \& Pav. & Herb & $\mathrm{F}$ & OUPR 1308 \\
\hline
\end{tabular}


MESSIAS, M.C.T.B. et al.

Table 1. Continued..

\begin{tabular}{|c|c|c|c|}
\hline Family / Species & Habit & Habitat & Voucher \\
\hline Bomarea edulis (Tussac) Herb. & Liana & $\mathrm{F}$ & OUPR 577 \\
\hline \multicolumn{4}{|l|}{ Amaranthaceae } \\
\hline Alternanthera brasiliana (L.) Kuntze & Subshrub & $\mathrm{F}$ & OUPR 6800 \\
\hline Alternanthera brasiliana var. moquini Uline \& W.L.Bray & Subshrub & $\mathrm{CR}, \mathrm{F}$ & RB 170197 \\
\hline Amaranthus spinosus L. & Subshrub & CR & RB 244325 \\
\hline Amaranthus viridis $\mathrm{L}$. & Subshrub & $\mathrm{AA}$ & NY 837913 \\
\hline Dysphania ambrosioides (L.) Mosyakin \& Clemants & Subshrub & $\mathrm{CR}$ & OUPR 2743 \\
\hline \multicolumn{4}{|l|}{ Amaryllidaceae } \\
\hline Hippeastrum correiense (Bury) Worsley & Herb & $\mathrm{CR}$ & RB 112160 \\
\hline Hippeastrum damazianum Beauverd & Herb & CR & OUPR 1404 \\
\hline Hippeastrum organense Herb. & Herb & $\mathrm{CR}$ & OUPR 7392 \\
\hline \multicolumn{4}{|l|}{ Anacardiaceae } \\
\hline Schinus terebinthifolius Raddi & Shrub & $\mathrm{CR}, \mathrm{F}$ & OUPR 1201 \\
\hline Tapirira guianensis Aubl. & Tree & $\mathrm{F}$ & OUPR 9059 \\
\hline \multicolumn{4}{|l|}{ Annonaceae } \\
\hline Annona emarginata (Schltdl.) H.Rainer & Tree & $\mathrm{F}$ & OUPR 19153 \\
\hline Guatteria australis A.St.-Hil. & Shrub & $\mathrm{F}$ & OUPR 20217 \\
\hline Guatteria pohliana Schltdl. VU* & Shrub & $\mathrm{F}$ & OUPR 5270 \\
\hline Guatteria sellowiana Schltdl. VU* & Tree & $\mathrm{F}$ & OUPR 1202 \\
\hline Guatteria villosissima A.St.-Hil. VU* & Tree & $\mathrm{F}$ & OUPR 1505 \\
\hline Xylopia brasiliensis Spreng. & Tree & $\mathrm{F}$ & OUPR 1398 \\
\hline \multicolumn{4}{|l|}{ Apiaceae } \\
\hline Centella asiatica (L.) Urb. & Herb & $\mathrm{CR}$ & OUPR 13609 \\
\hline Ciclospermum leptophyllum (Pers.) Britton P.Wilson & Subshrub & AA & NY 877641 \\
\hline Eryngium eurycephalum Malme & Herb & CR & NY 877731 \\
\hline Eryngium paniculatum Cav. \& Dombey ex F.Delaroche & Herb & CR & OUPR 1352 \\
\hline \multicolumn{4}{|l|}{ Apocynaceae } \\
\hline Asclepias curassavica $\mathrm{L}$. & Subshrub & CR & OUPR 19409 \\
\hline Aspidosperma spruceanum Benth. ex Müll.Arg. & Tree & $\mathrm{F}$ & OUPR 1468 \\
\hline Blepharodon ampliflorum E.Fourn. & Liana & $\mathrm{CR}$ & MO 1246283 \\
\hline Blepharodon pictum (Vahl) W.D.Stevens & Liana & $\mathrm{CR}$ & MO 1934634 \\
\hline Condylocarpon intermedium subsp. laxum (Müll.Arg.) Fallen & Liana & $\mathrm{F}$ & OUPR 366 \\
\hline Condylocarpon isthmicum (Vell.) A. DC. & Liana & CR & OUPR 17722 \\
\hline Ditassa aequicymosa E. Fourn. VU* & Liana & CR & RB 361320 \\
\hline Ditassa laevis Mart. VU*, EN** & Liana & $\mathrm{CR}$ & OUPR 833 \\
\hline Ditassa longisepala (Hua) Fontella \& E. A. Schwarz VU*, EN ${ }^{* *}$ & Liana & $\mathrm{CR}$ & OUPR 739 \\
\hline Ditassa mucronata Mart. & Liana & $\mathrm{F}$ & OUPR 1379 \\
\hline Ditassa nitida Decne. & Liana & $\mathrm{CR}$ & BHCB 110 \\
\hline Ditassa tomentosa (Decne.) Fontella & Liana & $\mathrm{CR}$ & OUPR 743 \\
\hline Forsteronia velloziana (A.DC.) Woodson & Liana & $\mathrm{F}$ & OUPR 6804 \\
\hline Gonioanthela hilariana (E. Fourn.) Malme & Liana & $\mathrm{CR}$ & OUPR 717 \\
\hline Mandevilla atroviolacea (Stadelm.) Woodson & Liana & $\mathrm{F}$ & RB 277734 \\
\hline Mandevilla emarginata (Vell.) C.Ezcurra & Shrub & $\mathrm{CR}$ & OUPR 1272 \\
\hline Mandevilla immaculata Woodson & Liana & $\mathrm{CR}$ & SP 139907 \\
\hline Mandevilla martiana (Stadelm.) Woodson & Liana & $\mathrm{CR}, \mathrm{F}$ & OUPR 400 \\
\hline Mandevilla sellowii (Müll.Arg.) Woodson & Liana & $\mathrm{F}$ & NY 1183021 \\
\hline Mandevilla tenuifolia (J.C.Mikan) Woodson & Subshrub & $\mathrm{CR}$ & OUPR 423 \\
\hline Mandevilla $\mathrm{sp} 1$ & Shrub & $\mathrm{CR}$ & RB 277638 \\
\hline Mandevilla sp2 & Subshrub & CR & OUPR 8261 \\
\hline Minaria decussata (Mart.) T.U.P.Konno \& Rapini & Liana & CR & OUPR 8743 \\
\hline Orthosia scoparia (Nutt.) Liede \& Meve & Liana & $\mathrm{F}$ & OUPR 6797 \\
\hline Oxypetalum appendiculatum Mart. & Liana & $\mathrm{CR}$ & OUPR 883 \\
\hline Oxypetalum foliosum Mart. & Subshrub & $\mathrm{CR}$ & OUPR 1488 \\
\hline Oxypetalum glabrum (Decne.) Malme & Liana & $\mathrm{CR}$ & BHCB 114 \\
\hline Oxypetalum insigne (Decne.) Malme & Liana & $\mathrm{CR}$ & OUPR 799 \\
\hline Oxypetalum minarum E. Fourn. & Liana & $\mathrm{CR}$ & OUPR 943 \\
\hline Oxypetalum strictum Mart. & Liana & $\mathrm{CR}$ & OUPR 7228 \\
\hline Peplonia asteria (Vell.) Fontella \& E.A.Schwarz & Liana & $\mathrm{F}$ & OUPR 717 \\
\hline
\end{tabular}


Table 1. Continued...

\begin{tabular}{|c|c|c|c|}
\hline Family / Species & Habit & Habitat & Voucher \\
\hline Stipecoma peltigera (Stadelm.) Müll. Arg. & Liana & $\mathrm{F}$ & OUPR 20151 \\
\hline \multicolumn{4}{|l|}{ Aquifoliaceae } \\
\hline Ilex chamaedryfolia Reissek & Shrub & $\mathrm{CR}$ & OUPR 478 \\
\hline Ilex conocarpa Reissek & Shrub & $\mathrm{CR}, \mathrm{F}$ & OUPR 13606 \\
\hline Ilex euryaeformis Reissek & Shrub & $\mathrm{CR}$ & OUPR 4639 \\
\hline Ilex grandis Reissek & Shrub & $\mathrm{F}$ & OUPR 1358 \\
\hline Ilex loranthoides Mart. ex Reissek VU*, & Shrub & $\mathrm{CR}$ & OUPR 1357 \\
\hline Ilex subcordata Reissek & Shrub & $\mathrm{CR}$ & OUPR 446 \\
\hline Ilex theezans Mart. ex Reissek & Tree & $\mathrm{F}$ & OUPR 19183 \\
\hline \multicolumn{4}{|l|}{ Araceae } \\
\hline Anthurium minarum Sakur. \& Mayo & Herb & $\mathrm{CR}$ & OUPR 1467 \\
\hline Anthurium scandens (Aubl.) Engl. & Herb & $\mathrm{CR}, \mathrm{F}$ & OUPR 1486 \\
\hline Philodendron propinquum Schott & Herb & $\mathrm{F}$ & OUPR 24872 \\
\hline \multicolumn{4}{|l|}{ Araliaceae } \\
\hline Dendropanax cuneatus (DC.) Decne \& Planch & Tree & $\mathrm{F}$ & OUPR 565 \\
\hline Hydrocotyle quinqueloba Ruiz \& Pav. & Herb & $\mathrm{CR}$ & OUPR 1504 \\
\hline Hydrocotyle verticillata Thunb. & Herb & $\mathrm{CR}$ & OUPR 1504 \\
\hline Schefflera calva (Cham.) Frodin \& Fiaschi & Tree & $\mathrm{F}$ & BHCB 8276 \\
\hline Schefflera macrocarpa (Cham. \& Schltdl.) Frodin & Tree & $\mathrm{CR}, \mathrm{F}$ & OUPR 534 \\
\hline Schefflera vinosa (Cham. \& Schltdl.) Frodin \& Fiaschi & Shrub & $\mathrm{F}$ & OUPR 1319 \\
\hline Schefflera $\mathrm{sp}$ & Shrub & $\mathrm{CR}$ & RB 112206 \\
\hline \multicolumn{4}{|l|}{ Araucariaceae } \\
\hline Araucaria angustifolia (Bertol.) Kuntze EN*,*** & Tree & $\mathrm{F}$ & OUPR 23543 \\
\hline \multicolumn{4}{|l|}{ Arecaceae } \\
\hline Geonoma brevispatha Barb.Rodr. & Palm & $\mathrm{F}$ & ВHCB 42284 \\
\hline Geonoma schottiana Mart. & Palm & $\mathrm{F}$ & OUPR 6955 \\
\hline \multicolumn{4}{|l|}{ Aristolochiaceae } \\
\hline Aristolochia smilacina (Klotzsch) Duch. & Liana & $\mathrm{CR}$ & OUPR 687 \\
\hline \multicolumn{4}{|l|}{ Asteraceae } \\
\hline Acanthospermum australe (Loefl.) Kuntze & Subshrub & AA & JB 490409 \\
\hline Achyrocline alata (Kunth) DC. & Subshrub & $\mathrm{CR}$ & OUPR 1547 \\
\hline Achyrocline albicans Griseb. & Subshrub & $\mathrm{CR}$ & OUPR 1553 \\
\hline Achyrocline satureioides (Lam.) DC. & Subshrub & $\mathrm{CR}$ & OUPR 12807 \\
\hline Ageratum conyzoides L. & Subshrub & $\mathrm{CR}$ & OUPR 1578 \\
\hline Ageratum fastigiatum (Gardner) R.M.King \& H.Rob. & Subshrub & $\mathrm{CR}$ & RB 486721 \\
\hline Ageratum myriadenium (Sch.Bip. ex Baker) R.M.King \& H.Rob. & Subshrub & $\mathrm{CR}$ & BHCB 41506 \\
\hline Apopyros corymbosus (Hook. \& Arn.) G.L.Nesom & Subshrub & $\mathrm{CR}$ & NY 787836 \\
\hline Aspilia caudata J.U.Santos CR* & Shrub & $\mathrm{CR}$ & VIC 26515 \\
\hline Aspilia pohlii (Sch.Bip. ex Baker) Baker DD ${ }^{* * *}$ & Subshrub & $\mathrm{CR}$ & VIC 8312 \\
\hline Austrocritonia angulicaulis (Sch. Bip. ex Baker) R.M.King \& H.Rob. & Subshrub & $\mathrm{CR}$ & OUPR 18551 \\
\hline Austrocritonia velutina (Gardner) R.M.King \& H.Rob. & Subshrub & $\mathrm{F}$ & ВНCB 161975 \\
\hline Austroeupatorium neglectum (B.L.Rob.) R.M.King \& H.Rob. & Shrub & $\mathrm{CR}$ & OUPR 14853 \\
\hline Ayapana amygdalina (Lam.) R.M.King \& H.Rob. & Subshrub & $\mathrm{CR}$ & OUPR 16892 \\
\hline Baccharis aphylla (Vell.) DC. & Subshrub & $\mathrm{CR}$ & OUPR 2320 \\
\hline Baccharis articulata (Lam.) Pers. & Subshrub & $\mathrm{CR}$ & VIC 22077 \\
\hline Baccharis brevifolia DC. & Subshrub & $\mathrm{CR}$ & RB 275538 \\
\hline Baccharis calvescens DC. & Shrub & $\mathrm{CR}$ & RB 486583 \\
\hline Baccharis cognata DC. & Subshrub & $\mathrm{CR}$ & RB 486604 \\
\hline Baccharis crispa Spreng. & Subshrub & $\mathrm{CR}$ & RB 486597 \\
\hline Baccharis dentata (Vell.) G.M.Barroso & Subshrub & $\mathrm{CR}$ & HUFU 42320 \\
\hline Baccharis dracunculifolia DC. & Subshrub & $\mathrm{CR}$ & OUPR 1287 \\
\hline Baccharis genistelloides (Lam.) Pers. & Subshrub & $\mathrm{CR}$ & OUPR 12791 \\
\hline Baccharis glutinosa Pers. & Subshrub & $\mathrm{CR}$ & VIC 22069 \\
\hline Baccharis gnidiifolia Kunth & Shrub & $\mathrm{CR}$ & OUPR 4937 \\
\hline Baccharis helichrysoides DC. & Shrub & $\mathrm{CR}$ & OUPR 1288 \\
\hline Baccharis hirta DC. & Subshrub & $\mathrm{CR}$ & OUPR 4852 \\
\hline Baccharis illinita DC. & Subshrub & $\mathrm{CR}$ & VIC22071 \\
\hline Baccharis junciformis DC. & Subshrub & CR & OUPR 4928 \\
\hline
\end{tabular}


MESSIAS, M.C.T.B. et al

Table 1. Continued..

\begin{tabular}{|c|c|c|c|}
\hline Family / Species & Habit & Habitat & Voucher \\
\hline Baccharis lateralis Baker & Shrub & CR & RB 157380 \\
\hline Baccharis latifolia (Ruiz \& Pav.) Pers. & Subshrub & CR & OUPR 3456 \\
\hline Baccharis ligustrina DC. & Shrub & $\mathrm{CR}$ & HUFU 42319 \\
\hline Baccharis linearifolia (Lam.) Pers. & Shrub & $\mathrm{CR}$ & VIC 22176 \\
\hline Baccharis lychnophora Gardner VU*, EN ${ }^{* *}$ & Shrub & $\mathrm{CR}$ & RB 275530 \\
\hline Baccharis microcephala (Less.) DC. & Subshrub & CR & VIC 22063 \\
\hline Baccharis montana DC. & Subshrub & $\mathrm{CR}$ & OUPR 1747 \\
\hline Baccharis oblongifolia (Ruiz \& Pav.) Pers & Subshrub & $\mathrm{CR}$ & RB 486562 \\
\hline Baccharis platypoda DC. & Shrub & CR & OUPR 9054 \\
\hline Baccharis pseudomyriocephala Malag. & Shrub & CR & OUPR 12806 \\
\hline Baccharis punctulata DC. & Shrub & $\mathrm{CR}$ & OUPR 3453 \\
\hline Baccharis reticularia DC. & Shrub & $\mathrm{CR}$ & OUPR 4865 \\
\hline Baccharis retusa DC. & Subshrub & $\mathrm{CR}$ & RB 486582 \\
\hline Baccharis rufidula (Spreng.) Joch.Müll. & Shrub & $\mathrm{CR}$ & RB 277732 \\
\hline Baccharis sagittalis (Less.) DC. & Subshrub & $\mathrm{CR}$ & OUPR 4928 \\
\hline Baccharis semiserrata DC. & Shrub & $\mathrm{CR}$ & RB 486706 \\
\hline Baccharis serrulata (Lam.) Pers. & Subshrub & $\mathrm{CR}$ & OUPR 23270 \\
\hline Baccharis sessiliflora Vahl & Shrub & $\mathrm{CR}$ & OUPR 2664 \\
\hline Baccharis subdentata DC. & Shrub & $\mathrm{CR}$ & RB 486595 \\
\hline Baccharis tarchonanthoides DC. & Subshrub & $\mathrm{CR}$ & OUPR 12790 \\
\hline Baccharis tridentata Vahl & Subshrub & $\mathrm{CR}$ & RB 486741 \\
\hline Baccharis trinervis Pers. & Liana & $\mathrm{CR}$ & RB 486620 \\
\hline Baccharis vulneraria Baker & Shrub & CR & OUPR 4994 \\
\hline Baccharis sp1 & Shrub & CR & OUPR 9053 \\
\hline Baccharis sp2 & Shrub & CR & OUPR 15602 \\
\hline Barrosoa organensis (Gardner) R.M.King \& H.Rob. & Shrub & $\mathrm{CR}$ & RB 486774 \\
\hline Bartlettina hemisphaerica (DC.) R.M.King \& H.Rob. & Shrub & CR & OUPR 14502 \\
\hline Bidens pilosa $\mathrm{L}$ & Subshrub & CR & OUPR 13594 \\
\hline Bidens rubifolia Kunth & Liana & $\mathrm{CR}, \mathrm{F}$ & OUPR 1354 \\
\hline Bidens segetum Mart. ex Colla & Liana & $\mathrm{F}$ & VIC 26510 \\
\hline Calea clausseniana Baker & Subshrub & $\mathrm{CR}$ & VIC 26502 \\
\hline Calea clematidea Baker & Subshrub & $\mathrm{CR}$ & OUPR 22365 \\
\hline Calea fruticosa (Gardner) Urbatsch, Zlotsky \& Pruski & Shrub & $\mathrm{CR}$ & OUPR 1286 \\
\hline Calea lemmatioides Sch.Bip. ex Baker & Subshrub & $\mathrm{CR}$ & OUPR 1338 \\
\hline Calea rotundifolia (Less.) Baker & Subshrub & $\mathrm{CR}$ & RB 489982 \\
\hline Campovassouria cruciata (Vell.) R.M.King \& H.Rob. & Shrub & $\mathrm{CR}$ & OUPR 16909 \\
\hline Centratherum punctatum Cass. & Subshrub & $\mathrm{CR}$ & OUPR 20305 \\
\hline Chaptalia graminifolia (Dusén) Cabrera & Subshrub & $\mathrm{CR}$ & VIC 21840 \\
\hline Chaptalia integerrima (Vell.) Burkart & Subshrub & $\mathrm{CR}$ & RB 324851 \\
\hline Chaptalia martii (Baker) Zardini EN* & Subshrub & $\mathrm{CR}$ & OUPR 7038 \\
\hline Chaptalia nutans (L.) Pol. & Subshrub & $\mathrm{CR}$ & OUPR 13583 \\
\hline Chaptalia runcinata Kunth & Subshrub & $\mathrm{CR}$ & NY 362359 \\
\hline Chevreulia acuminata Less. & Subshrub & $\mathrm{CR}$ & RB 490161 \\
\hline Chionolaena capitata (Baker) S.E.Freire & Subshrub & $\mathrm{CR}$ & RB 1468 \\
\hline Chionolaena lychnophorioides Sch.Bip. VU* & Subshrub & $\mathrm{CR}$ & RB 324850 \\
\hline Chromolaena adenolepis (Sch.Bip. ex Baker) R.M.King \& H.Rob. & Subshrub & CR & VIC 26568 \\
\hline Chromolaena barbacensis (Hieron.) R.M.King \& H.Rob. & Subshrub & CR & RB 486723 \\
\hline Chromolaena cylindrocephala (Sch.Bip. ex Baker) R.M.King \& H.Rob. & Subshrub & $\mathrm{CR}$ & RB 275393 \\
\hline Chromolaena decumbens Gardner & Subshrub & $\mathrm{CR}$ & OUPR 1341 \\
\hline Chromolaena laevigata (Lam.) R.M.King \& H.Rob. & Shrub & $\mathrm{CR}$ & OUPR 18318 \\
\hline Chromolaena latisquamulosa (Hieron.) R.M.King \& H.Rob & Subshrub & $\mathrm{CR}$ & RB 489213 \\
\hline Chromolaena maximilianii (Schrad. ex DC.) R.M.King \& H.Rob. & Subshrub & $\mathrm{CR}$ & OUPR 18517 \\
\hline Chromolaena multiflosculosa (DC.) R.M.King \& H.Rob. & Subshrub & $\mathrm{CR}$ & OUPR 18843 \\
\hline Chromolaena odorata (L.) R.M.King \& H.Rob. & Shrub & $\mathrm{AA}, \mathrm{CR}$ & OUPR 14460 \\
\hline Chromolaena pedalis (Sch. Bip. ex Baker) R.M.King \& H.Rob. & Subshrub & $\mathrm{CR}$ & OUPR 1343 \\
\hline Chromolaena perforata (Sch.Bip. ex Baker) R.M.King \& H.Rob. & Subshrub & $\mathrm{CR}$ & RB 486781 \\
\hline Chromolaena squalida (DC.) R.M.King \& H.Rob. & Subshrub & $\mathrm{CR}$ & OUPR 16856 \\
\hline Chromolaena squarrulosa (Hook. \& Arn.) R.M.King \& H.Rob. & Subshrub & $\mathrm{CR}$ & OUPR 18567 \\
\hline
\end{tabular}


Table 1. Continued...

\begin{tabular}{|c|c|c|c|}
\hline Family / Species & Habit & Habitat & Voucher \\
\hline Chromolaena stachyophylla (Spreng.) R.M.King \& H.Rob. & Subshrub & CR & OUPR 15603 \\
\hline Chromolaena vindex (DC.) R.M.King \& H.Rob. & Subshrub & $\mathrm{CR}$ & RB 486779 \\
\hline Chrysolaena cognata (Less.) Dematt. & Subshrub & $\mathrm{CR}$ & OUPR 19959 \\
\hline Chrysolaena simplex (Less.) Dematt. & Subshrub & $\mathrm{CR}$ & OUPR 3776 \\
\hline Chrysolaena obovata (Less.) Dematt. & Subshrub & $\mathrm{CR}$ & HUFU 54022 \\
\hline Chrysolaena platensis (Spreng.) H.Rob. & Subshrub & $\mathrm{CR}$ & OUPR 20752 \\
\hline Chrysolaena propringua (Hieron.) H.Rob. & Subshrub & $\mathrm{CR}$ & RB 158177 \\
\hline Chrysolaena verbascifolia (Less.) H.Rob. & Subshrub & $\mathrm{F}$ & OUPR 2939 \\
\hline Clibadium armani (Balb.) Sch. Bip. ex O.E. Schulz & Subshrub & $\mathrm{CR}$ & OUPR 1458 \\
\hline Conyza bonariensis (L.) Cronquist & Subshrub & $\mathrm{CR}$ & RB 486589 \\
\hline Conyza canadensis (L.) Cronquist & Subshrub & AA & VIC 22160 \\
\hline Conyza monorchis (Griseb.) Cabrera & Subshrub & $\mathrm{CR}$ & RB 162919 \\
\hline Conyza primulifolia (Lam.) Cuatrec. \& Lourteig & Subshrub & $\mathrm{F}$ & RB 486622 \\
\hline Conyza sumatrensis (Retz.) E.Walker & Subshrub & $\mathrm{F}$ & RB 486608 \\
\hline Cyrtocymura scorpioides (Lam.) H.Rob. & Subshrub & $\mathrm{CR}$ & OUPR 13602 \\
\hline Dasyphyllum brasiliense (Spreng.) Cabrera & Shrub & $\mathrm{CR}$ & RB 468820 \\
\hline Dasyphyllum candolleanum (Gardner) Cabrera & Shrub & $\mathrm{CR}$ & VIC 21807 \\
\hline Dasyphyllum flagellare (Casar.) Cabrera. & Shrub & $\mathrm{CR}$ & OUPR 1401 \\
\hline Dasyphyllum fodinarum (Gardner) Cabrera & Shrub & $\mathrm{CR}$ & OUPR 5152 \\
\hline Dasyphyllum sprengelianum var. inerme (Gardner) Cabrera & Shrub & CR & VIC 21799 \\
\hline Dasyphyllum sprengelianum var. sprengelianum (Gardner) Cabrera & Shrub & $\mathrm{CR}$ & VIC 21796 \\
\hline Dendrophorbium pellucidinerve (Sch.Bip. ex Baker) C.Jeffrey & Shrub & $\mathrm{CR}$ & OUPR 15109 \\
\hline Echinocoryne holosericea (Mart. ex DC.) H.Rob. & Subshrub & CR & OUPR 20145 \\
\hline Echinocoryne schwenkiifolia (Mart. ex DC.) H.Rob. & Subshrub & $\mathrm{CR}$ & VIC 21974 \\
\hline Eclipta prostrata (L.) L. & Subshrub & $\mathrm{AA}, \mathrm{CR}$ & OUPR 14333 \\
\hline Elephantopus biflorus (Less.) Sch.Bip. & Subshrub & $\mathrm{CR}$ & OUPR 14344 \\
\hline Elephantopus mollis Kunth & Herb & $\mathrm{AA}, \mathrm{CR}$ & OUPR 14353 \\
\hline Emilia fosbergii Nicolson & Subshrub & $\mathrm{CR}$ & VIC 22225 \\
\hline Emilia sonchifolia (L.) DC. ex Wight & Subshrub & $\mathrm{AA}, \mathrm{CR}$ & OUPR 2166 \\
\hline Erechtites hieracifolius (L.) Raf. ex DC. & Subshrub & $\mathrm{CR}$ & OUPR 14372 \\
\hline Erechtites valerianifolius (Wolf) DC & Subshrub & $\mathrm{CR}$ & OUPR 14380 \\
\hline Eremanthus crotonoides (DC.) Sch.Bip. & Shrub & $\mathrm{CR}$ & OUPR 19248 \\
\hline Eremanthus erythropappus (DC.) MacLeish & Tree & $\mathrm{CR}, \mathrm{F}$ & OUPR 1385 \\
\hline Eremanthus glomerulatus Less. & Tree & $\mathrm{CR}$ & VIC 21891 \\
\hline Eremanthus incanus (Less.) Less. & Shrub & $\mathrm{CR}$ & OUPR 16943 \\
\hline Fleischmannia laxa (Gardner) R.M. King \& H. Rob. & Subshrub & $\mathrm{CR}$ & OUPR 14028 \\
\hline Galatella linosyris (L.) Rchb.f. & Subshrub & $\mathrm{CR}$ & OUPR 2080 \\
\hline Galinsoga parviflora Cav. & Subshrub & AA & VIC 26498 \\
\hline Gamochaeta americana (Mill.) Wedd. & Subshrub & $\mathrm{CR}$ & RB 490033 \\
\hline Gamochaeta purpurea (L.) Cabrera & Subshrub & CR & RB 490162 \\
\hline Gamochaeta simplicicaulis (Willd. ex Spreng.) Cabrera & Subshrub & $\mathrm{CR}$ & RB 163267 \\
\hline Gamochaeta $\mathrm{sp}$ & Subshrub & $\mathrm{CR}$ & RB 190526 \\
\hline Gnaphalium cheiranthifolium Bertero ex Lam. & Subshrub & $\mathrm{CR}$ & OUPR 14961 \\
\hline Grazielia coriacea (Scheele) R.M.King \& H.Rob. & Subshrub & $\mathrm{CR}$ & OUPR 14457 \\
\hline Grazielia gaudichaudeana (DC.) R.M.King \& H.Rob. & Shrub & CR & RB 163258 \\
\hline Grazielia intermedia (DC.) R.M.King \& H.Rob. & Shrub & $\mathrm{CR}$ & RB 486734 \\
\hline Grazielia serrata (Spreng.) R.M.King \& H.Rob. & Subshrub & $\mathrm{CR}$ & OUPR 18489 \\
\hline Guayania yaviana (Lasser \& Maguire) R.M.King \& H.Rob. & Shrub & $\mathrm{CR}$ & OUPR 18551 \\
\hline Heterocondylus alatus (Vell.) R.M.King \& H.Rob. & Shrub & $\mathrm{AA}, \mathrm{CR}$ & RB 486729 \\
\hline Heterocondylus amphidictyus (DC.) R.M.King \& H.Rob. & Subshrub & CR & OUPR 1337 \\
\hline Heterocondylus pumilus (Gardner) R.M.King \& H.Rob. & Subshrub & $\mathrm{CR}$ & RB 277730 \\
\hline Hololepis pedunculata (DC. ex Pers.) DC. VU* & Shrub & $\mathrm{CR}$ & OUPR 1389 \\
\hline Hoehnephytum trixoides (Gardner) Cabrera VU* & Subshrub & $\mathrm{CR}$ & RB 324847 \\
\hline Hypochaeris chillensis (Kunth) Hieron. & Herb & $\mathrm{AA}, \mathrm{CR}$ & OUPR 14940 \\
\hline Hypochaeris gardneri Baker & Herb & $\mathrm{CR}$ & VIC 21875 \\
\hline Inulopsis scaposa (DC.) O.Hoffm. EN* & Herb & CR & OUPR 8353 \\
\hline Jaegeria hirta (Lag.) Less. & Herb & $\mathrm{AA}, \mathrm{CR}$ & VIC 26495 \\
\hline Koanophyllon adamantium (Gardner) R.M. King \& H. Rob. EN* & Subshrub & CR & OUPR 14308 \\
\hline
\end{tabular}


Table 1. Continued..

\begin{tabular}{|c|c|c|c|}
\hline Family / Species & Habit & Habitat & Voucher \\
\hline Koanophyllon consanguineum (DC.) R.M.King \& H.Rob. & Subshrub & CR & OUPR 14464 \\
\hline Koanophyllon thysanolepis (B.L.Rob.) R.M.King \& H.Rob. & Shrub & $\mathrm{CR}$ & RB 163210 \\
\hline Lepidaploa araripensis (Gardner) H.Rob. & Liana & $\mathrm{CR}$ & OUPR 20101 \\
\hline Lepidaploa argyrotricha (Sch.Bip. ex Baker) H.Rob. & Subshrub & $\mathrm{CR}$ & HUFU 54025 \\
\hline Lepidaploa cotoneaster (Willd. ex Spreng.) H.Rob. & Liana & $\mathrm{F}$ & OUPR 19247 \\
\hline Lepidaploa helophila (Mart. ex DC.) H.Rob. & Shrub & CR & VIC 21934 \\
\hline $\begin{array}{l}\text { Lepidaploa lilacina (Mart. ex DC.) H.Rob. VU* (=Vernonia adamantium } \\
\text { Gardner) }\end{array}$ & Subshrub & $\mathrm{CR}$ & OUPR 19432 \\
\hline Lepidaploa muricata (DC.) H.Rob. & Subshrub & $\mathrm{CR}$ & VIC 21917 \\
\hline Lepidaploa persericea (H.Rob.) H.Rob. & Subshrub & $\mathrm{CR}$ & VIC 21916 \\
\hline Lepidaploa remotiflora (Rich.) H.Rob. & Shrub & CR & OUPR 24656 \\
\hline Lepidaploa rufogrisea (A. St.-Hil.) H.Rob. & Subshrub & $\mathrm{CR}, \mathrm{F}$ & RB 275396 \\
\hline Lepidaploa salzmannii (DC.) H.Rob. & Subshrub & CR & OUPR 2920 \\
\hline Lepidaploa sororia (DC.) H.Rob. & Subshrub & $\mathrm{CR}$ & RB 56519 \\
\hline Lepidaploa vauthieriana (DC.) H.Rob. & Subshrub & $\mathrm{CR}$ & OUPR 14559 \\
\hline Leptostelma maximum D.Don & Subshrub & $\mathrm{CR}$ & OUPR 1336 \\
\hline Lessingianthus brevipetiolatus (Sch.Bip. ex Baker) H.Rob. & Shrub & $\mathrm{CR}, \mathrm{F}$ & HUFU 54037 \\
\hline Lessingianthus linearifolius (Less.) H.Rob. & Subshrub & $\mathrm{CR}$ & OUPR 4131 \\
\hline Lessingianthus obscurus (Less.) H.Rob. & Shrub & $\mathrm{CR}$ & OUPR 20146 \\
\hline Lessingianthus psilophyllus (DC.) H.Rob. & Subshrub & $\mathrm{CR}$ & RB 324843 \\
\hline Lessingianthus rubricaulis (Humb. \& Bonpl.) H.Rob. & Subshrub & $\mathrm{CR}$ & HUFU 61540 \\
\hline Lessingianthus syncephalus (Sch.Bip. ex Baker) H.Rob. & Subshrub & $\mathrm{CR}$ & OUPR 1339 \\
\hline Lessingianthus tomentellus (Mart. ex DC.) H.Rob. & Subshrub & $\mathrm{CR}$ & OUPR 1335 \\
\hline Lessingianthus vepretorum (Mart. ex DC.) H.Rob. & Subshrub & $\mathrm{CR}$ & RB 490166 \\
\hline Lessingianthus virgulatus (Mart. ex DC.) H.Rob. & Subshrub & $\mathrm{CR}$ & OUPR 2932 \\
\hline Lucilia linearifolia Baker & Subshrub & $\mathrm{CR}$ & VIC 22005 \\
\hline Lucilia lycopodioides (Less.) S.E.Freire & Subshrub & $\mathrm{CR}$ & OUPR 1507 \\
\hline Lychnophora ericoides Mart. VU*, $\mathrm{NT}^{* *}$ & Shrub & $\mathrm{CR}$ & OUPR 13498 \\
\hline Lychnophora passerina (Mart. ex DC.) Gardner VU* & Shrub & $\mathrm{CR}$ & BHCB 318 \\
\hline Lychnophora pinaster Mart. VU*, $\mathrm{NT}^{* * *}$ & Shrub & $\mathrm{CR}$ & OUPR 9057 \\
\hline Lychnophora reticulata Gardner $\mathrm{EN}^{* *}$ & Shrub & $\mathrm{CR}$ & HUFU 54028 \\
\hline Melampodium divaricatum (Rich. ex Pers.) DC & Subshrub & $\mathrm{CR}$ & VIC 26494 \\
\hline Mikania acuminata DC. & Liana & $\mathrm{F}$ & BHCB 54647 \\
\hline Mikania argyreiae DC. VU*, & Liana & $\mathrm{CR}$ & OUPR 24668 \\
\hline Mikania badiniana G.S.S. Almeida \& Carvalho-Okano & Subshrub & $\mathrm{CR}$ & VIC 29114 \\
\hline Mikania banisteriae DC. & Liana & CR & OUPR 5941 \\
\hline Mikania candolleana Gardner & Liana & $\mathrm{CR}$ & OUPR 24669 \\
\hline Mikania capricorni B.L. Rob. & Liana & $\mathrm{F}$ & OUPR 1332 \\
\hline Mikania clematidifolia Dusén VU** & Shrub & $\mathrm{F}$ & VIC 27606 \\
\hline Mikania duckei G.M. Barroso & Liana & $\mathrm{CR}$ & OUPR 14646 \\
\hline Mikania glauca Mart. ex Baker EN",** & Subshrub & CR & BHCB 41476 \\
\hline Mikania hemisphaerica Sch.Bip. ex Baker & Liana & $\mathrm{CR}$ & HUFU 39918 \\
\hline Mikania hirsutissima DC. & Liana & CR & RB 98143 \\
\hline Mikania lasiandrae DC. & Liana & $\mathrm{CR}, \mathrm{F}$ & BHCB 41501 \\
\hline Mikania lindbergii Baker & Liana & $\mathrm{CR}$ & OUPR 14706 \\
\hline Mikania microcephala DC. & Liana & $\mathrm{CR}$ & VIC 28777 \\
\hline Mikania microdonta DC. & Liana & $\mathrm{CR}$ & RB 324845 \\
\hline Mikania microphylla Sch.Bip. & Subshrub & $\mathrm{CR}$ & BHCB 56217 \\
\hline Mikania microptera DC. & Liana & $\mathrm{F}$ & OUPR 15006 \\
\hline Mikania nummularia DC. & Subshrub & $\mathrm{CR}$ & OUPR 16755 \\
\hline Mikania oblongifolia DC. & Subshrub & CR & OUPR 14758 \\
\hline Mikania obtusata DC. & Subshrub & $\mathrm{CR}$ & RB 162942 \\
\hline Mikania officinalis Mart. & Subshrub & $\mathrm{CR}$ & VIC 28764 \\
\hline Mikania parviflora (Aubl.) H.Karst. & Liana & $\mathrm{F}$ & OUPR 14618 \\
\hline Mikania parvifolia Baker & Subshrub & $\mathrm{CR}$ & BHCB 50618 \\
\hline Mikania phaeoclados Mart. & Liana & $\mathrm{CR}$ & VIC 28754 \\
\hline Mikania premnifolia Gardner $\mathrm{VU}^{*}, \mathrm{EN}^{* *}$ & Subshrub & $\mathrm{CR}$ & OUPR 14768 \\
\hline Mikania psilostachya DC. & Liana & $\mathrm{F}$ & VIC 8250 \\
\hline
\end{tabular}


Table 1. Continued...

\begin{tabular}{|c|c|c|c|}
\hline Family / Species & Habit & Habitat & Voucher \\
\hline Mikania ramosissima Gardner & Shrub & $\mathrm{CR}$ & OUPR 15002 \\
\hline Mikania reticulata Gardner & Shrub & $\mathrm{CR}$ & ВНCB 161990 \\
\hline Mikania scandens (L.) Willd. & Liana & $\mathrm{F}$ & OUPR 15006 \\
\hline Mikania schenckii Hieron. & Liana & $\mathrm{CR}$ & VIC 28752 \\
\hline Mikania selloi Spreng. & Liana & $\mathrm{F}$ & OUPR 15015 \\
\hline Mikania sericea Hook. \& Arn. & Liana & $\mathrm{F}$ & VIC 28750 \\
\hline Mikania sessilifolia DC. & Subshrub & $\mathrm{CR}$ & BHCB 54693 \\
\hline Mikania subverticillata Baker & Subshrub & $\mathrm{CR}$ & OUPR 14809 \\
\hline Mikania ternata (Vell.) B.L.Rob. & Liana & $\mathrm{F}$ & BHCB 8210 \\
\hline Mikania testudinaria DC. & Liana & $\mathrm{F}$ & BHCB 152825 \\
\hline Mikania trinervis Hook. \& Arn. & Liana & $\mathrm{F}$ & BHCB 54658 \\
\hline Mikania vauthieriana Baker & Liana & $\mathrm{F}$ & BHCB 152826 \\
\hline Mikania vismiifolia DC. & Liana & $\mathrm{CR}$ & OUPR 15046 \\
\hline Mikania warmingii Sch.Bip. ex Baker EN* & Shrub & $\mathrm{CR}$ & VIC 29120 \\
\hline Mikania $\mathrm{sp} 1$ & Shrub & $\mathrm{CR}$ & OUPR 1879 \\
\hline Mikania sp2 & Liana & $\mathrm{F}$ & OUPR 16754 \\
\hline Mikania sp3 & Subshrub & $\mathrm{CR}$ & OUPR 7281 \\
\hline Mikania sp4 & Subshrub & $\mathrm{CR}$ & OUPR 15604 \\
\hline Mikania sp5 & Subshrub & $\mathrm{CR}$ & OUPR 16600 \\
\hline Moquinia racemosa (Spreng.) DC. & Shrub & $\mathrm{CR}$ & RB 324853 \\
\hline Moquiniastrum densicephalum (Cabrera) G.Sancho & Subshrub & $\mathrm{CR}$ & HUFU 54032 \\
\hline Moquiniastrum floribundum (Cabrera) G.Sancho & Shrub & $\mathrm{CR}$ & RB 162938 \\
\hline Moquiniastrum paniculatum (Less.) G.Sancho & Shrub & $\mathrm{CR}$ & OUPR 14886 \\
\hline Moquiniastrum polymorphum (Less.) G.Sancho & Shrub & $\mathrm{F}$ & HUFU 54040 \\
\hline Moquiniastrum pulchrum (Cabrera) G.Sancho & Shrub & $\mathrm{CR}$ & VIC 21818 \\
\hline Mutisia campanulata Less. & Liana & $\mathrm{CR}$ & OUPR 1208 \\
\hline Mutisia speciosa Aiton ex Hook. & Shrub & $\mathrm{CR}$ & VIC 21817 \\
\hline Ophryosporus freyreysii (Thunb.) Baker & Subshrub & $\mathrm{CR}$ & RB 145118 \\
\hline Orthopappus angustifolius (Sw.) Gleason & Subshrub & $\mathrm{CR}$ & HUFU 54018 \\
\hline Pentacalia desiderabilis (Vell.) Cuatrec. & Liana & $\mathrm{F}$ & OUPR 16117 \\
\hline Piptocarpha axillaris (Less.) Baker & Tree & $\mathrm{F}$ & HUFU 54024 \\
\hline Piptocarpha macropoda (DC.) Baker & Tree & $\mathrm{F}$ & OUPR 19150 \\
\hline Piptocarpha oblonga (Gardner) Baker & Shrub & $\mathrm{F}$ & OUPR 16027 \\
\hline Piptocarpha regnellii (Sch.Bip.) Cabrera & Tree & $\mathrm{F}$ & OUPR 16039 \\
\hline Piptocarpha tomentosa Baker & Tree & $\mathrm{F}$ & OUPR 5771 \\
\hline Piptocarpha $\mathrm{sp}$ & Tree & $\mathrm{F}$ & OUPR 1206 \\
\hline Piptolepis ericoides Sch. Bip. VU* & Subshrub & $\mathrm{CR}, \mathrm{F}$ & OUPR 6815 \\
\hline Piptolepis sp & Subshrub & $\mathrm{CR}$ & OUPR 1457 \\
\hline Pluchea oblongifolia DC. & Subshrub & $\mathrm{CR}$ & RB 163287 \\
\hline Pluchea sagittalis (Lam.) Cabrera & Subshrub & $\mathrm{AA}, \mathrm{CR}$ & OUPR 16061 \\
\hline Porophyllum ruderale (Jacq.) Cass. & Subshrub & $\mathrm{AA}, \mathrm{CR}$ & VIC 26523 \\
\hline Praxelis kleinioides (Kunth) Sch. Bip. & Subshrub & $\mathrm{CR}$ & RB 486724 \\
\hline Praxelis pauciflora (Kunth) R.M.King \& H.Rob. & Subshrub & $\mathrm{CR}$ & VIC 27577 \\
\hline Pseudobrickellia brasiliensis (Spreng.) R.M.King \& H.Rob. & Subshrub & $\mathrm{CR}$ & OUPR 5095 \\
\hline Pseudognaphalium cheiranthifolium (Lam.) Hilliard \& Burtt & Subshrub & $\mathrm{CR}$ & RB 490164 \\
\hline Pterocaulon alopecuroides (Lam.) DC. & Subshrub & $\mathrm{AA}, \mathrm{CR}$ & OUPR 15097 \\
\hline Pterocaulon balansae Chodat & Subshrub & $\mathrm{CR}$ & VIC 22023 \\
\hline Pterocaulon rugosum (Vahl) Malme & Subshrub & $\mathrm{CR}$ & VIC 22021 \\
\hline Pterocaulon $\mathrm{sp}$ & Subshrub & $\mathrm{CR}$ & OUPR 23275 \\
\hline Raulinoreitzia crenulata (Spreng.) R.M.King \& H.Rob. & Shrub & $\mathrm{CR}$ & OUPR 14447 \\
\hline Richterago campestris Roque \& J.N. Nakaj. EN* & Herb & $\mathrm{CR}$ & VIC 21866 \\
\hline Richterago discoidea (Less.) Kuntze & Subshrub & $\mathrm{CR}$ & OUPR 14903 \\
\hline Richterago hatschbachii (Zardini) Roque EN* & Herb & $\mathrm{CR}$ & OUPR 23296 \\
\hline Richterago petiolata Roque \& J.N.Nakaj. EN* & Herb & $\mathrm{CR}$ & VIC 21862 \\
\hline Richterago polymorpha (Less.) Roque & Herb & $\mathrm{CR}$ & HUFU 54033 \\
\hline Richterago radiata (Vell.) Roque & Herb & $\mathrm{CR}$ & OUPR 1453 \\
\hline Richterago sp & Herb & $\mathrm{CR}$ & OUPR 3143 \\
\hline Senecio adamantinus Bong. & Subshrub & $\mathrm{CR}$ & OUPR 20458 \\
\hline
\end{tabular}


MESSIAS, M.C.T.B. et al.

Table 1. Continued..

\begin{tabular}{|c|c|c|c|}
\hline Family / Species & Habit & Habitat & Voucher \\
\hline Senecio brasiliensis (Spreng.) Less. & Shrub & CR & OUPR 21503 \\
\hline Senecio colpodes Bong. & Subshrub & $\mathrm{CR}$ & RB 490410 \\
\hline Senecio pohlii Sch.Bip. ex Baker VU* & Subshrub & $\mathrm{CR}$ & BHCB118650 \\
\hline Sonchus asper (L.) Hill & Herb & AA & VIC 21873 \\
\hline Sonchus oleraceus L. & Herb & AA & VIC 21871 \\
\hline Sphagneticola trilobata (L.) Pruski & Subshrub & $\mathrm{CR}$ & OUPR 21504 \\
\hline Stenocephalum megapotamicum (Spreng.) Sch.Bip. & Subshrub & $\mathrm{CR}$ & RB 56531 \\
\hline Stenocline chionaea (ex de Candolle) DC. & Shrub & $\mathrm{CR}$ & OUPR 16188 \\
\hline Stenophalium chionaeum (DC.) Anderb. & Subshrub & CR & RB 487378 \\
\hline Stenophalium gardneri (Baker) D.J.N.Hind & Subshrub & $\mathrm{CR}$ & BHCB125649 \\
\hline Stevia alexii G.S.S.Almeida \& Carvalho-Okano & Subshrub & $\mathrm{CR}$ & VIC 29113 \\
\hline Stevia camporum Baker & Subshrub & $\mathrm{CR}$ & VIC 29116 \\
\hline Stevia claussenii Sch.Bip. ex Baker & Subshrub & $\mathrm{CR}$ & OUPR 1374 \\
\hline Stevia hilarii B.L.Rob. VU*, ${ }^{*} R^{* *}$ & Subshrub & $\mathrm{CR}$ & VIC 29111 \\
\hline Stevia myriadenia Sch.Bip. ex Baker & Subshrub & $\mathrm{CR}$ & VIC 29110 \\
\hline Stevia resinosa Gardner & Subshrub & $\mathrm{CR}$ & RB 98089 \\
\hline Stevia urticaefolia Thunb. & Subshrub & $\mathrm{CR}$ & OUPR 24672 \\
\hline Symphyopappus angustifolius Cabrera & Shrub & $\mathrm{CR}$ & RB 110427 \\
\hline Symphyopappus compressus (Gardner) B.L.Rob. & Shrub & $\mathrm{CR}$ & OUPR 1481 \\
\hline Symphyopappus cuneatus (DC.) Sch.Bip. ex Baker & Shrub & $\mathrm{CR}$ & BHCB 137583 \\
\hline Symphyopappus itatiayensis (Hieron.) R.M.King \& H.Rob. & Shrub & $\mathrm{CR}$ & VIC 28810 \\
\hline Symphyopappus reticulatus Baker & Shrub & $\mathrm{CR}$ & OUPR 1480 \\
\hline Symphyotrichum regnellii (Baker) G.L.Nesom & Subshrub & $\mathrm{CR}$ & VIC 22231 \\
\hline Symphyotrichum squamatum (Spreng.) G.L.Nesom & Subshrub & $\mathrm{CR}$ & RB 486586 \\
\hline Tagetes minuta $\mathrm{L}$. & Subshrub & CR & OUPR 12812 \\
\hline Trichogonia eupatorioides (Gardner) R.M.King \& H.Rob. EX* & Subshrub & $\mathrm{CR}$ & OUPR 17246 \\
\hline Trichogonia hirtiflora (DC.) Sch. Bip. ex Baker & Shrub & $\mathrm{CR}$ & OUPR 17207 \\
\hline Trichogonia salviifolia Gardner & Subshrub & $\mathrm{CR}$ & OUPR 1344 \\
\hline Trichogonia villosa (Spreng.) Sch.Bip. ex Baker & Subshrub & $\mathrm{CR}$ & OUPR 1345 \\
\hline Trixis antimenorrhoea (Schrank) Kuntze & Shrub & CR & OUPR 18367 \\
\hline Trixis glaziovii Baker VU*** & Subshrub & $\mathrm{CR}$ & HUFU 54041 \\
\hline Trixis lessingii DC. & Subshrub & $\mathrm{CR}$ & OUPR 7386 \\
\hline Trixis nobilis (Vell.) Katinas & Subshrub & CR & OUPR 1456 \\
\hline Trixis verbascifolia (Gardner) Blake & Subshrub & CR & OUPR 15255 \\
\hline Trixis sp & Subshrub & $\mathrm{CR}$ & OUPR 1455 \\
\hline Verbesina glabrata Hook \& Arn. & Shrub & $\mathrm{CR}$ & OUPR 1451 \\
\hline Verbesina luetzelburgii Mattf. & Shrub & $\mathrm{CR}$ & VIC 26488 \\
\hline Vernonanthura discolor (Spreng.) H.Rob. & Tree & $\mathrm{F}$ & OUPR 7430 \\
\hline Vernonanthura lindbergii (Baker) H. Rob. & Subshrub & $\mathrm{CR}$ & OUPR 20099 \\
\hline Vernonanthura montevidensis (Spreng.) H.Rob. & Subshrub & CR & OUPR 19249 \\
\hline Vernonanthura mucronulata (Less.) H.Rob. & Subshrub & $\mathrm{CR}$ & VIC 21919 \\
\hline Vernonanthura phosphorica (Vell.) H.Rob. & Shrub & $\mathrm{CR}$ & BHCB41489 \\
\hline Vernonanthura viscidula (Less.) H.Rob. & Shrub & $\mathrm{CR}$ & OUPR 2932 \\
\hline Vernonanthura westiniana (Less.) H.Rob. & Shrub & $\mathrm{CR}$ & OUPR 2948 \\
\hline Vittetia orbiculata (DC.) R.M.King \& H.Rob. & Subshrub & $\mathrm{CR}$ & BHCB118661 \\
\hline Willoughbya acuminata (DC.) Kuntze & Liana & $\mathrm{F}$ & OUPR 14619 \\
\hline Willoughbya candolleana (Gardner) Kuntze & Liana & $\mathrm{CR}$ & OUPR 14608 \\
\hline Willoughbya estrellensis (Baker) Kuntze & Liana & $\mathrm{CR}$ & OUPR 14644 \\
\hline Willoughbya glauca (Mart.) Kuntze & Subshrub & $\mathrm{CR}$ & OUPR 1333 \\
\hline Willoughbya lasiandra (DC.) Kuntze & Liana & $\mathrm{CR}$ & OUPR 1334 \\
\hline Willoughbya leiolaena DC. & Subshrub & $\mathrm{CR}$ & RB 362360 \\
\hline Willoughbya microdonta (DC.) Kuntze & Liana & $\mathrm{CR}$ & RB 324845 \\
\hline Willoughbya oblongifolia (DC.) Kuntze & Shrub & $\mathrm{CR}$ & RB 163216 \\
\hline Willoughbya obtusata (DC.) Kuntze & Subshrub & $\mathrm{CR}$ & OUPR 15610 \\
\hline Willoughbya ramosissima (Gardner) Kuntze & Liana & $\mathrm{F}$ & OUPR 3142 \\
\hline Willoughbya selloi (Spreng.) Kuntze & Liana & $\mathrm{F}$ & OUPR 15015 \\
\hline Willoughbya sessilifolia (DC.) Kuntze & Shrub & $\mathrm{CR}$ & OUPR 14793 \\
\hline Willoughbya subverticillata (Sch. Bip. ex Baker) Kuntze & Subshrub & $\mathrm{CR}$ & OUPR 14809 \\
\hline
\end{tabular}


Table 1. Continued...

\begin{tabular}{|c|c|c|c|}
\hline Family / Species & Habit & Habitat & Voucher \\
\hline Willoughbya vismiifolia (DC.) Kuntze & Liana & CR & OUPR 15046 \\
\hline \multicolumn{4}{|l|}{ Balanophoraceae } \\
\hline Langsdorffia hypogaea Mart. & Herb & $\mathrm{F}$ & OUPR 987 \\
\hline \multicolumn{4}{|l|}{ Balsaminaceae } \\
\hline Impatiens balsamina $\mathrm{L}$. & Subshrub & AA & OUPR 19303 \\
\hline \multicolumn{4}{|l|}{ Begoniaceae } \\
\hline Begonia cucullata Willd. & Subshrub & CR & NY 877944 \\
\hline Begonia rufa Thunb. & Subshrub & CR & OUPR 9090 \\
\hline Begonia valdensium A.DC. & Subshrub & CR & RB 47524 \\
\hline \multicolumn{4}{|l|}{ Bignoniaceae } \\
\hline Adenocalymma bracteatum (Cham.) DC. & Liana & $\mathrm{F}$ & VIC 30460 \\
\hline Adenocalymma cymbalum (Cham.) Bureau \& K.Schum. & Liana & $\mathrm{F}$ & VIC 30459 \\
\hline Adenocalymma magnoalatum Scud. EN* $\mathrm{CR}^{* *}$ & Liana & $\mathrm{F}$ & VIC 30454 \\
\hline Adenocalymma marginatum (Cham.) DC. & Liana & $\mathrm{F}$ & VIC 30451 \\
\hline Adenocalymma subsessilifolium DC. & Liana & $\mathrm{F}$ & VIC 30448 \\
\hline Adenocalymma ternatum (Vell.) Mello ex Bureau \& K.Schum. & Shrub & $\mathrm{F}$ & OUPR 1429 \\
\hline Adenocalymma sp & Liana & $\mathrm{CR}$ & OUPR 1141 \\
\hline Amphilophium crucigerum (L.) L.G.Lohmann & Liana & $\mathrm{F}$ & VIC 29400 \\
\hline Amphilophium elongatum (Vahl) L.G.Lohmann & Liana & $\mathrm{F}$ & VIC 30376 \\
\hline Amphilophium paniculatum (L.) Kunth & Liana & $\mathrm{F}$ & VIC 30447 \\
\hline Anemopaegma arvense (Vell.) Stellfeld ex de Souza EN** & Subshrub & CR & VIC 30445 \\
\hline Anemopaegma chamberlaynii (Sims) Bureau \& K. Schum. & Liana & $\mathrm{F}$ & OUPR 1034 \\
\hline Anemopaegma floridum Mart. ex DC. & Liana & $\mathrm{F}$ & VIC 30442 \\
\hline Anemopaegma prostratum DC. & Liana & CR & OUPR 7513 \\
\hline Anemopaegma setilobum A.H.Gentry & Shrub & CR & VIC 30424 \\
\hline Bignonia binata Thunb. & Liana & $\mathrm{F}$ & VIC 21770 \\
\hline Bignonia costata (Bureau \& K.Schum.) L.G.Lohmann & Liana & $\mathrm{F}$ & VIC 30384 \\
\hline Bignonia prieurii DC. & Liana & $\mathrm{F}$ & VIC 29404 \\
\hline Callichlamys latifolia (Rich.) K.Schum. & Liana & $\mathrm{F}$ & VIC 30393 \\
\hline Cuspidaria floribunda (DC.) A.H.Gentry & Liana & $\mathrm{F}$ & VIC 30380 \\
\hline Cybistax antisyphilitica (Mart.) Mart. & Tree & $\mathrm{F}$ & VIC 30378 \\
\hline Dolichandra quadrivalvis (Jacq.) L.G.Lohmann & Liana & $\mathrm{F}$ & VIC 29405 \\
\hline Dolichandra unguiculata (Vell.) L.G.Lohmann & Liana & $\mathrm{F}$ & VIC 29402 \\
\hline Dolichandra unguis-cati (L.) L.G.Lohmann & Liana & $\mathrm{F}$ & VIC 29411 \\
\hline Fridericia candicans (Rich.) L.G.Lohmann & Liana & $\mathrm{F}$ & VIC 30417 \\
\hline Fridericia florida (DC.) L.G.Lohmann & Liana & $\mathrm{F}$ & VIC 30415 \\
\hline Fridericia formosa (Bureau) L.G.Lohmann & Liana & $\mathrm{F}$ & VIC 30410 \\
\hline Fridericia platyphylla (Cham.) L.G.Lohmann & SubShrub & $\mathrm{CR}$ & VIC 30418 \\
\hline Fridericia pubescens (L.) L.G.Lohmann & Liana & $\mathrm{F}$ & VIC 30408 \\
\hline Fridericia rego (Vell.) L.G.Lohmann & Liana & $\mathrm{F}$ & VIC 30401 \\
\hline Fridericia samydoides (Cham.) L.G.Lohmann & Liana & $\mathrm{CR}, \mathrm{F}$ & OUPR 1297 \\
\hline Fridericia speciosa Mart. & Liana & CR & OUPR 1329 \\
\hline Fridericia triplinervia (Mart. ex DC.) L.G.Lohmann & Liana & $\mathrm{F}$ & VIC 30398 \\
\hline Fridericia tynanthoides (A.H.Gentry) L.G.Lohmann & Liana & $\mathrm{F}$ & VIC 30395 \\
\hline Handroanthus albus (Cham.) Mattos VU* & Tree & $\mathrm{F}$ & OUPR 19280 \\
\hline Handroanthus bureavii (Sandwith) S.Grose & Tree & $\mathrm{CR}, \mathrm{F}$ & VIC 29442 \\
\hline Handroanthus chrysotrichus (Mart. ex DC.) Mattos & Tree & $\mathrm{F}$ & VIC 29435 \\
\hline Handroanthus vellosoi (Toledo) Mattos & Tree & $\mathrm{F}$ & VIC 29431 \\
\hline Jacaranda macrantha Cham. & Tree & $\mathrm{F}$ & VIC 29248 \\
\hline Jacaranda mimosifolia D.Don & Tree & $\mathrm{F}$ & OUPR 8202 \\
\hline Jacaranda montana Morawetz & Tree & $\mathrm{F}$ & VIC 29427 \\
\hline Jacaranda puberula Cham. & Tree & $\mathrm{F}$ & VIC 29425 \\
\hline Lundia cordata (Vell.) DC. & Liana & $\mathrm{F}$ & VIC 29424 \\
\hline Lundia corymbifera (Vahl) Sandwith & Liana & $\mathrm{F}$ & VIC 29416 \\
\hline Manaosella cordifolia (DC.) A.H.Gentry & Liana & $\mathrm{F}$ & VIC 29409 \\
\hline Pleonotoma stichadenia K.Schum. & Liana & $\mathrm{F}$ & VIC 29399 \\
\hline Pleonotoma tetraquetra (Cham.) Bureau & Liana & $\mathrm{F}$ & VIC 29398 \\
\hline Pyrostegia venusta (Ker Gawl.) Miers & Liana & $\mathrm{CR}, \mathrm{F}$ & OUPR 1134 \\
\hline
\end{tabular}


Table 1. Continued..

\begin{tabular}{|c|c|c|c|}
\hline Family / Species & Habit & Habitat & Voucher \\
\hline Sparattosperma leucanthum (Vell.) K. Schum. & Tree & F & OUPR 20259 \\
\hline Stizophyllum aff. inaequilaterum Bureau \& K.Schum. & Liana & $\mathrm{F}$ & VIC 29392 \\
\hline Stizophyllum perforatum (Cham.) Miers & Liana & $\mathrm{F}$ & VIC 29391 \\
\hline Stizophyllum riparium (Kunth) Sandwith & Liana & $\mathrm{F}$ & VIC 29280 \\
\hline Tabebuia heterophylla (DC.) Britton & Liana & $\mathrm{F}$ & VIC 29390 \\
\hline Tabebuia nodosa (Griseb.) Griseb. & Tree & $\mathrm{F}$ & OUPR 20259 \\
\hline Tecoma stans (L.) Juss. ex Kunth & Shrub & AA & OUPR 19374 \\
\hline Tynanthus fasciculatus (Vell.) Miers & Liana & $\mathrm{F}$ & VIC 29388 \\
\hline Xylophragma myrianthum (Cham. ex Steud.) Sprague & Liana & $\mathrm{F}$ & VIC 29386 \\
\hline Zeyheria montana Mart. & Shrub & $\mathrm{CR}$ & VIC 29384 \\
\hline \multicolumn{4}{|l|}{ Bixaceae } \\
\hline Bixa orellana $\mathrm{L}$. & Shrub & F & OUPR 1361 \\
\hline \multicolumn{4}{|l|}{ Boraginaceae } \\
\hline Cordia curassavica (Jacq.) Roem. \& Schult. & Subshrub & $\mathrm{CR}$ & OUPR 3211 \\
\hline Cordia sellowiana Cham. & Tree & $\mathrm{CR}, \mathrm{F}$ & OUPR 4812 \\
\hline Heliotropium arborescens $\mathrm{L}$. & Shrub & AA & OUPR 1203 \\
\hline \multicolumn{4}{|l|}{ Bromeliaceae } \\
\hline Acanthostachys strobilacea (Schult. \& Schult.f.) Klotzsch & Epiphyte & $\mathrm{CR}$ & VIC 37212 \\
\hline Aechmea bromeliifolia (Rudge) Baker var. bromeliifolia & Herb & $\mathrm{CR}$ & VIC 21067 \\
\hline Aechmea lamarchei $\mathrm{Mez}$ & Epiphyte & $\mathrm{F}$ & VIC 21069 \\
\hline Aechmea nudicaulis var. aureorosea (Antoine) L.B.Sm. & Herb & $\mathrm{CR}$ & VIC 21070 \\
\hline Aechmea sp & Herb & $\mathrm{CR}$ & OUPR 13880 \\
\hline Billbergia elegans Mart. ex Schult. \& Schult.f. & Herb & $\mathrm{CR}, \mathrm{F}$ & OUPR 20235 \\
\hline Billbergia minarum L.B.Sm. & Herb & $\mathrm{CR}$ & RB 258593 \\
\hline Billbergia vittata Brongn. & Herb & $\mathrm{CR}$ & VIC 21090 \\
\hline Cryptanthus schwackeanus Mez. & Herb & $\mathrm{CR}$ & VIC 21098 \\
\hline Dyckia bracteata (Wittm.) Mez & Herb & $\mathrm{CR}$ & RB 112248 \\
\hline Dyckia cinerea $\mathrm{Mez}$ & Herb & $\mathrm{CR}$ & VIC 27818 \\
\hline Dyckia saxatilis $\mathrm{Mez}$ & Herb & $\mathrm{CR}$ & VIC 27806 \\
\hline Dyckia schwackeana $\mathrm{Mez}$ & Herb & $\mathrm{CR}$ & RB 275535 \\
\hline Dyckia trichostachya Baker & Herb & $\mathrm{CR}$ & RB 275531 \\
\hline Neoregelia mucugensis Leme & Herb & $\mathrm{CR}$ & VIC 21104 \\
\hline Nidularium marigoi Leme & Herb & $\mathrm{CR}$ & VIC 21107 \\
\hline Pitcairnia flammea Lindl. var. flammea & Herb & $\mathrm{CR}$ & VIC 27803 \\
\hline Pseudananas sagenarius (Arruda) Camargo & Herb & $\mathrm{CR}$ & VIC 21108 \\
\hline Racinaea aerisincola $(\mathrm{Mez})$ M.A.Spencer \& L.B.Sm. & Epiphyte & $\mathrm{CR}$ & VIC 23656 \\
\hline Tillandsia pohliana $\mathrm{Mez}$ & Epiphyte & $\mathrm{F}$ & OUPR 14301 \\
\hline Tillandsia polystachia (L.) L. & Epiphyte & $\mathrm{CR}$ & VIC (T.S. Coser et al. 24) \\
\hline Tillandsia stricta Sol. & Epiphyte & $\mathrm{F}$ & VIC 27798 \\
\hline Vriesea bituminosa Wawra & Herb & $\mathrm{CR}$ & VIC 23657 \\
\hline Vriesea clausseniana (Baker) Mez VU* & Herb & $\mathrm{CR}$ & VIC 27793 \\
\hline Vriesea hoehneana L.B.Sm. & Herb & $\mathrm{CR}$ & VIC 27785 \\
\hline Vriesea minarum L.B.Sm. VU*, EN** & Herb & $\mathrm{CR}$ & VIC 36491 \\
\hline Vriesea minor (L.B.Sm.) Leme & Herb & $\mathrm{CR}$ & RB 112301 \\
\hline Vriesea regnellii Mez. & Herb & $\mathrm{CR}$ & VIC 26453 \\
\hline Vriesea $\mathrm{sp1}$ & Herb & $\mathrm{CR}$ & VIC 26457 \\
\hline Vriesea $\mathrm{sp} 2$ & Herb & $\mathrm{CR}$ & VIC 26458 \\
\hline \multicolumn{4}{|l|}{ Burmanniaceae } \\
\hline Burmannia damazii Beauverd EN* & Herb & $\mathrm{CR}$ & OUPR 13042 \\
\hline Burmannia sp & Herb & $\mathrm{CR}$ & OUPR 13471 \\
\hline \multicolumn{4}{|l|}{ Burseraceae } \\
\hline Protium brasiliense (Spreng.) Engl. & Tree & F & OUPR 2452 \\
\hline \multicolumn{4}{|l|}{ Cactaceae } \\
\hline Hatiora salicornioides (Haw.) Britton \& Rose & Epiphyte & $\mathrm{CR}, \mathrm{F}$ & OUPR 7431 \\
\hline Rhipsalis floccosa subsp. pulvinigera (Lindb.) Barthlott \& N.P.Taylor & Herb & $\mathrm{CR}$ & OUPR 20568 \\
\hline \multicolumn{4}{|l|}{ Campanulaceae } \\
\hline Centropogon cornutus (L.) Druce & Subshrub & $\mathrm{CR}$ & OUPR 1204 \\
\hline Lobelia camporum Pohl. & Subshrub & $\mathrm{CR}$ & OUPR 13425 \\
\hline
\end{tabular}


Table 1. Continued...

\begin{tabular}{|c|c|c|c|}
\hline Family / Species & Habit & Habitat & Voucher \\
\hline Lobelia thapsoidea Schott & Subshrub & $\mathrm{CR}$ & RB 97999 \\
\hline Siphocampylus nitidus Pohl. & Subshrub & $\mathrm{CR}$ & OUPR 12800 \\
\hline Siphocampylus verticillatus (Cham.) G. Don & Subshrub & $\mathrm{F}$ & OUPR 1264 \\
\hline Siphocampylus westinianus (Thunb.) Pohl & Subshrub & $\mathrm{CR}$ & BHCB 8249 \\
\hline Siphocampylus $\mathrm{sp} 1$ & Subshrub & $\mathrm{CR}$ & OUPR 5058 \\
\hline Siphocampylus $\mathrm{sp} 2$ & Subshrub & $\mathrm{F}$ & OUPR 6825 \\
\hline Siphocampylus sp3 & Subshrub & $\mathrm{F}$ & OUPR 6826 \\
\hline \multicolumn{4}{|l|}{ Cannabaceae } \\
\hline Trema micrantha (L.) Blume & Tree & $\mathrm{F}$ & OUPR 20241 \\
\hline \multicolumn{4}{|l|}{ Caprifoliaceae } \\
\hline Lonicera japonica Thunb. & Shrub & AA & OUPR 19304 \\
\hline Valeriana organensis Gardner $\mathrm{CR}^{* *}$ & Liana & $\mathrm{F}$ & OUPR 1798 \\
\hline Valeriana scandens $\mathrm{L}$. & Liana & $\mathrm{F}$ & OUPR 24838 \\
\hline \multicolumn{4}{|l|}{ Celastraceae } \\
\hline Maytenus longifolia Reissek ex Loes & Shrub & $\mathrm{F}$ & RB 398994 \\
\hline Maytenus salicifolia Reissek & Subshrub & $\mathrm{F}$ & OUPR 18916 \\
\hline Peritassa campestris (Cambess.) A.C.Sm. & Subshrub & $\mathrm{F}$ & BHCB 41525 \\
\hline \multicolumn{4}{|l|}{ Chloranthaceae } \\
\hline Hedyosmum brasiliense Mart. ex Miq. & Shrub & $\mathrm{CR}, \mathrm{F}$ & OUPR 1441 \\
\hline \multicolumn{4}{|l|}{ Chrysobalanaceae } \\
\hline Couepia grandiflora (Mart. \& Zucc.) Benth. & Shrub & $\mathrm{F}$ & OUPR 1431 \\
\hline Hirtella floribunda Cham. \& Schltdl. & Liana & $\mathrm{CR}$ & OUPR 20274 \\
\hline Licania humilis Cham. \& Schltdl. & Shrub & $\mathrm{F}$ & OUPR 22222 \\
\hline \multicolumn{4}{|l|}{ Clethraceae } \\
\hline Clethra scabra Pers. & Tree & $\mathrm{CR}, \mathrm{F}$ & OUPR 1275 \\
\hline \multicolumn{4}{|l|}{ Clusiaceae } \\
\hline Clusia arrudea Planch. \& Triana & Shrub & $\mathrm{CR}$ & NY 1022677 \\
\hline Clusia fluminensis Planch. \& Triana & Shrub & $\mathrm{CR}$ & OUPR 1360 \\
\hline Clusia spathulifolia Engl. & Shrub & $\mathrm{CR}, \mathrm{F}$ & OUPR 20150 \\
\hline \multicolumn{4}{|l|}{ Commelinaceae } \\
\hline Commelina diffusa Burm.f. & Herb & $\mathrm{CR}$ & MO 3020454 \\
\hline Commelina $\mathrm{sp}$ & Herb & $\mathrm{CR}$ & OUPR 17721 \\
\hline Dichorisandra pubescens Mart. ex Schult \& Schult.f. & Herb & $\mathrm{CR}, \mathrm{F}$ & OUPR 27241 \\
\hline Dichorisandra thyrsiflora J.C.Mikan & Subshrub & $\mathrm{CR}$ & OUPR 22794 \\
\hline Dichorisandra sp & Herb & $\mathrm{CR}$ & OUPR 16390 \\
\hline Gibasis geniculate (Jacq.) Rohweder & Herb & $\mathrm{CR}$ & OUPR 1414 \\
\hline Tripogandra diuretica (Mart.) Handlos & Herb & $\mathrm{CR}$ & OUPR 9063 \\
\hline Tripogandra serrulata (Vahl) Handlos & Herb & $\mathrm{F}$ & OUPR 1413 \\
\hline \multicolumn{4}{|l|}{ Convolvulaceae } \\
\hline Ipomoea cairica $(\mathrm{L}$.) Sweet & Liana & $\mathrm{CR}$ & OUPR 19301 \\
\hline Ipomoea delphinioides Choisy & Liana & $\mathrm{CR}, \mathrm{F}$ & OUPR 1210 \\
\hline Ipomoea indica (Burm.f.) Merr. & Liana & $\mathrm{AA}, \mathrm{F}$ & BHCB 41509 \\
\hline Ipomoea purpurea (L.) Roth & Liana & $\mathrm{CR}, \mathrm{F}$ & OUPR 1370 \\
\hline Jacquemontia densiflora (Meisn.) Hallier f. & Liana & $\mathrm{F}$ & OUPR 8250 \\
\hline Jacquemontia ferruginea Choisy & Liana & $\mathrm{F}$ & HRCB 41557 \\
\hline Jacquemontia prostrata Choisy & Liana & $\mathrm{CR}$ & OUPR 3022 \\
\hline Jacquemontia rufa (Choisy) Hallier f. & Liana & $\mathrm{CR}$ & BHCB 41512 \\
\hline Merremia contorquens (Choisy) Hallier $\mathrm{f}$. & Liana & $\mathrm{F}$ & BHCB 41520 \\
\hline Merremia macrocalyx (Ruiz \& Pav.) O’Donell & Liana & $\mathrm{CR}, \mathrm{F}$ & OUPR 1406 \\
\hline Merremia repens D.F.Austin $\mathrm{EN}^{* * *}$ & Liana & $\mathrm{F}$ & NY 1014441 \\
\hline Merremia sp & Liana & $\mathrm{F}$ & OUPR 1465 \\
\hline Turbina corymbosa (L.) Raf. & Liana & $\mathrm{F}$ & OUPR 7746 \\
\hline \multicolumn{4}{|l|}{ Cucurbitaceae } \\
\hline Cayaponia sp & Liana & $\mathrm{CR}$ & OUPR 5378 \\
\hline Melothrianthus smilacifolius (Cogn.) M.Crov. & Liana & $\mathrm{F}$ & OUPR 9973 \\
\hline \multicolumn{4}{|l|}{ Cunoniaceae } \\
\hline Lamanonia cuneata (Cambess.) Kuntze & Tree & $\mathrm{F}$ & OUPR 1397 \\
\hline Lamanonia ternata Vell. & Tree & $\mathrm{F}$ & OUPR 4807 \\
\hline
\end{tabular}


Table 1. Continued..

\begin{tabular}{|c|c|c|c|}
\hline Family / Species & Habit & Habitat & Voucher \\
\hline Weinmannia paulliniifolia Pohl ex Ser. & Subshrub & $\mathrm{CR}$ & BHCB 460 \\
\hline Weinmannia pinnata $\mathrm{L}$. & Subshrub & $\mathrm{CR}$ & OUPR 1396 \\
\hline \multicolumn{4}{|l|}{ Cyperaceae } \\
\hline Ascolepis brasiliensis (Kunth) Benth. ex C.B.Clarke & Herb & $\mathrm{F}$ & OUPR 1411 \\
\hline Bulbostylis capillaris (L.) C.B.Clarke & Herb & $\mathrm{CR}, \mathrm{F}$ & OUPR 12582 \\
\hline Bulbostylis consanguinea (Kunth) C.B.Clarke & Herb & $\mathrm{CR}$ & NY 916633 \\
\hline Bulbostylis hirtella (Schrad.) Urb. & Herb & $\mathrm{CR}$ & NY 916717 \\
\hline Bulbostylis junciformis (Kunth) C.B.Clarke & Herb & $\mathrm{CR}$ & OUPR 1502 \\
\hline Bulbostylis juncoides (Vahl) Kük. & Herb & $\mathrm{CR}$ & NY 916717 \\
\hline Bulbostylis paradoxa (Spreng.) Lindm. & Herb & $\mathrm{CR}$ & OUPR 12505 \\
\hline Bulbostylis sphaerocephala (Boeckeler) C.B.Clarke & Herb & $\mathrm{CR}$ & MO 2941369 \\
\hline Bulbostylis vestita (Kunth) C.B.Clarke & Herb & $\mathrm{CR}$ & NY918765 \\
\hline Cryptangium claussenii C.B.Clarke & Herb & $\mathrm{CR}$ & OUPR 13058 \\
\hline Cryptangium junciforme (Kunth) Boeckeler & Herb & $\mathrm{CR}$ & OUPR 13071 \\
\hline Cryptangium minarum (Nees) Boeckeler & Herb & $\mathrm{CR}$ & OUPR 13072 \\
\hline Cyperus aggregatus (Willd.) Endl. & Herb & $\mathrm{CR}$ & NY 918974 \\
\hline Cyperus gardneri Nees & Herb & $\mathrm{CR}$ & NY 919012 \\
\hline Cyperus haspan $\mathrm{L}$. & Herb & $\mathrm{CR}$ & NY 919056 \\
\hline Cyperus odoratus $\mathrm{L}$. & Herb & $\mathrm{CR}$ & OUPR 15239 \\
\hline Cyperus pohlii (Nees) Steud. & Herb & $\mathrm{CR}$ & OUPR 15945 \\
\hline Cyperus rotundus $\mathrm{L}$. & Herb & $\mathrm{CR}$ & OUPR 15240 \\
\hline Cyperus simplex Kunth & Herb & $\mathrm{CR}$ & BHCB 483 \\
\hline Cyperus sp & Herb & $\mathrm{CR}$ & OUPR 17495 \\
\hline Eleocharis debilis Kunth & Herb & $\mathrm{CR}$ & NY 925594 \\
\hline Eleocharis filiculmis Kunth & Herb & $\mathrm{CR}$ & NY 925630 \\
\hline Eleocharis sellowiana Kunth & Herb & $\mathrm{CR}$ & NY 925702 \\
\hline Fimbristylis complanata (Retz.) Link & Herb & $\mathrm{CR}$ & NY 925764 \\
\hline Fimbristylis dichotoma (L.) Vahl & Herb & $\mathrm{CR}$ & UB 163367 \\
\hline Kyllinga brevifolia Rottb. & Herb & $\mathrm{CR}$ & NY 926309 \\
\hline Lagenocarpus rigidus Nees & Herb & $\mathrm{CR}$ & OUPR 7294 \\
\hline Lipocarpha micrantha (Vahl) G.C.Tucker & Herb & $\mathrm{CR}$ & OUPR 1502 \\
\hline Pycreus lanceolatus (Poir.) C.B.Clarke & Herb & $\mathrm{CR}$ & NY 1112699 \\
\hline Rhynchospora albobracteata A.C.Araújo & Herb & $\mathrm{CR}$ & OUPR 15953 \\
\hline Rhynchospora brasiliensis Boeckeler & Herb & $\mathrm{CR}$ & OUPR 17499 \\
\hline Rhynchospora ciliata Kük. & Herb & $\mathrm{CR}$ & OUPR 12463 \\
\hline Rhynchospora consanguinea (Kunth) Boeckeler & Herb & $\mathrm{CR}$ & RB 287166 \\
\hline Rhynchospora corymbosa (L.) Britton & Herb & $\mathrm{CR}$ & OUPR 16609 \\
\hline Rhynchospora cryptantha C.B.Clarke & Herb & $\mathrm{CR}$ & OUPR 13059 \\
\hline Rhynchospora emaciata (Nees) Boeckeler & Herb & $\mathrm{CR}$ & NY 938535 \\
\hline Rhynchospora polyphylla (Vahl) Vahl & Herb & $\mathrm{CR}$ & OUPR 15953 \\
\hline Rhynchospora rugosa subsp. brownii (Roem. \& Schult.) T.Koyama & Herb & $\mathrm{CR}$ & OUPR 17703 \\
\hline Rhynchospora speciosa (Kunth) Boeckeler & Herb & $\mathrm{CR}$ & OUPR 17736 \\
\hline Rhynchospora tenuis Link & Herb & $\mathrm{CR}$ & OUPR 15963 \\
\hline Rhynchospora sp1 & Herb & $\mathrm{CR}$ & OUPR 10019 \\
\hline Rhynchospora sp2 & Herb & $\mathrm{CR}$ & OUPR 17736 \\
\hline Rhynchospora sp3 & Herb & $\mathrm{CR}$ & OUPR 11503 \\
\hline Rhynchospora sp4 & Herb & $\mathrm{CR}$ & OUPR 23279 \\
\hline Scleria sp & Herb & $\mathrm{CR}$ & OUPR 22769 \\
\hline \multicolumn{4}{|l|}{ Dilleniaceae } \\
\hline Davilla angustifolia A. St.-Hil. & Liana & $\mathrm{CR}$ & OUPR 1331 \\
\hline Davilla rugosa Poir & Liana & $\mathrm{F}$ & OUPR 1318 \\
\hline \multicolumn{4}{|l|}{ Dioscoreaceae } \\
\hline Dioscorea debilis Uline ex R.Knuth & Liana & $\mathrm{CR}$ & BHCB 8232 \\
\hline Dioscorea demourae Uline ex R.Knuth & Liana & $\mathrm{F}$ & OUPR 1824 \\
\hline Dioscorea grisebachii Kunth & Liana & $\mathrm{F}$ & RB 324861 \\
\hline Dioscorea monadelpha (Kunth) Griseb. & Liana & $\mathrm{CR}$ & OUPR 1824 \\
\hline Dioscorea ovata Vell. & Liana & $\mathrm{CR}$ & OUPR $13382 \mathrm{~A}$ \\
\hline Dioscorea piperifolia Humb. \& Bonpl. ex Willd. & Liana & $\mathrm{CR}$ & OUPR 13382B \\
\hline
\end{tabular}


Table 1. Continued...

\begin{tabular}{|c|c|c|c|}
\hline Family / Species & Habit & Habitat & Voucher \\
\hline Dioscorea schwakei Uline ex Knuth & Liana & $\mathrm{CR}$ & RB 61004 \\
\hline \multicolumn{4}{|l|}{ Droseraceae } \\
\hline Drosera communis A.St.-Hil. & Herb & $\mathrm{CR}$ & OUPR 9071 \\
\hline Drosera latifolia (Eichler) Gonella \& Rivadavia & Herb & $\mathrm{CR}$ & NY 918650 \\
\hline Drosera montana A.St.-Hil. & Herb & $\mathrm{CR}$ & OUPR 12788 \\
\hline Drosera villosa A.St.-Hil. & Herb & $\mathrm{CR}$ & RB 116710 \\
\hline \multicolumn{4}{|l|}{ Ebenaceae } \\
\hline Diospyros kaki Thunb. & Tree & $\mathrm{AA}$ & OUPR 22827 \\
\hline Diospyros sp & Shrub & $\mathrm{F}$ & OUPR 9067 \\
\hline \multicolumn{4}{|l|}{ Elaeocarpaceae } \\
\hline Sloanea hirsuta (Schott) Planch. ex Benth. & Tree & $\mathrm{F}$ & OUPR 18921 \\
\hline \multicolumn{4}{|l|}{ Ericaceae } \\
\hline Agarista coriifolia var. bradei (Sleumer) Judd & Subshrub & $\mathrm{CR}$ & RB 182938 \\
\hline Agarista oleifolia (Cham.) G.Don & Shrub & $\mathrm{F}$ & OUPR 6807 \\
\hline Agarista pulchella Cham. ex G.Don & Tree & $\mathrm{F}$ & OUPR 19288 \\
\hline Agarista pulchella var. cordifolia (Meisn.) Judd & Shrub & $\mathrm{CR}$ & OUPR 4581 \\
\hline Gaultheria eriophylla (Pers.) Sleumer ex Burtt & Subshrub & $\mathrm{CR}$ & OUPR 12793 \\
\hline Gaylussacia chamissonis Meisn. & Shrub & $\mathrm{CR}$ & OUPR 8247 \\
\hline Gaylussacia decipiens Cham. & Subshrub & $\mathrm{CR}$ & OUPR 13626 \\
\hline Gaylussacia densa Cham. & Subshrub & $\mathrm{CR}$ & OUPR 6026 \\
\hline Gaylussacia gardneri Meisn. & Shrub & $\mathrm{CR}$ & NY 943288 \\
\hline Gaylussacia incana Cham. & Subshrub & $\mathrm{CR}$ & OUPR 6025 \\
\hline Gaylussacia pallida Cham. & Subshrub & $\mathrm{CR}$ & RB 139389 \\
\hline Gaylussacia pinifolia Cham. \& Schltdl. & Shrub & $\mathrm{CR}$ & OUPR 4183 \\
\hline Gaylussacia pseudogaultheria Cham. \& Schltd & Subshrub & $\mathrm{CR}$ & BHCB 162557 \\
\hline Gaylussacia reticulata Mart. ex Meisn. & Shrub & $\mathrm{CR}, \mathrm{F}$ & OUPR 1294 \\
\hline Gaylussacia rugosa Cham. \& Schltdl. & Shrub & $\mathrm{CR}$ & OUPR 6817 \\
\hline Gaylussacia salicifolia Sleumer & Shrub & $\mathrm{CR}$ & OUPR 12789 \\
\hline Leucothoe ambigua Mart. & Shrub & $\mathrm{CR}$ & OUPR 7286 \\
\hline Leucothoe laxiflora Meiss. & Subshrub & $\mathrm{CR}$ & OUPR 1384 \\
\hline Rhododendron simsii Planch. & Subshrub & AA & OUPR 21502 \\
\hline \multicolumn{4}{|l|}{ Eriocaulaceae } \\
\hline Actinocephalus bongardii (A.St.-Hil.) Sano & Herb & $\mathrm{CR}$ & BHCB 134051 \\
\hline Comanthera nivea (Bong.) L.R.Parra \& Giul. & Herb & $\mathrm{CR}$ & R 3711 \\
\hline Eriocaulon ligulatum (Vell.) L.B.Sm. & Herb & $\mathrm{CR}$ & OUPR 6981 \\
\hline Leiothrix flavescens (Bong.) Ruhland & Herb & $\mathrm{CR}$ & OUPR 13145 \\
\hline Leiothrix vivipara (Bong.) Ruhland & Herb & $\mathrm{CR}$ & OUPR 5741 \\
\hline Paepalanthus aequalis (Vell.) J.F.Macbr. & Herb & $\mathrm{CR}$ & OUPR 13184 \\
\hline Paepalanthus ciliolatus Ruhland & Herb & $\mathrm{CR}$ & OUPR 4569 \\
\hline Paepalanthus conduplicatus Körn. & Herb & $\mathrm{CR}$ & OUPR 13193 \\
\hline Paepalanthus elongatus (Bong.) Körn. & Herb & $\mathrm{CR}$ & OUPR 12561 \\
\hline Paepalanthus exiguus (Bong.) Körn. & Herb & $\mathrm{CR}$ & OUPR 12572 \\
\hline Paepalanthus flaccidus (Bong.) Kunth & Herb & $\mathrm{CR}$ & NY 897855 \\
\hline Paepalanthus freyreissii (Thunb.) Körn. & Herb & $\mathrm{CR}$ & OUPR 12589 \\
\hline Paepalanthus mollis Kunth & Herb & $\mathrm{CR}$ & В 100243890 \\
\hline Paepalanthus planifolius (Bong.) Körn. & Herb & $\mathrm{CR}$ & OUPR 1212 \\
\hline Paepalanthus plantagineus (Bong.) Körn. & Herb & $\mathrm{CR}$ & OUPR 13286 \\
\hline Syngonanthus caulescens (Poir.) Ruhland & Herb & $\mathrm{CR}$ & OUPR 13348 \\
\hline \multicolumn{4}{|l|}{ Erythroxylaceae } \\
\hline Erythroxylum campestre A.St.-Hil. & Shrub & $\mathrm{CR}$ & OUPR 6038 \\
\hline Erythroxylum gonocladum (Mart.) O.E.Schulz & Subshrub & $\mathrm{F}$ & OUPR 10776 \\
\hline Erythroxylum microphyllum A.St.-Hil. & Subshrub & $\mathrm{CR}$ & BHCB 574 \\
\hline Erythroxylum sp1 & Shrub & $\mathrm{CR}$ & RB 324862 \\
\hline Erythroxylum sp2 & Shrub & $\mathrm{CR}$ & RB 324863 \\
\hline \multicolumn{4}{|l|}{ Escalloniaceae } \\
\hline Escallonia hispida (Vell.) Sleumer & Shrub & $\mathrm{CR}$ & OUPR 1255 \\
\hline \multicolumn{4}{|l|}{ Euphorbiaceae } \\
\hline Alchornea glandulosa subsp. iricurana (Casar.) Secco & Shrub & $\mathrm{F}$ & OUPR 7429 \\
\hline
\end{tabular}


Table 1. Continued..

\begin{tabular}{|c|c|c|c|}
\hline Family / Species & Habit & Habitat & Voucher \\
\hline Alchornea triplinervia (Spreng.) Müll. Arg. & Tree & $\mathrm{CR}, \mathrm{F}$ & OUPR 9061 \\
\hline Croton argyroglossus Baill. & Shrub & $\mathrm{F}$ & OUPR 9058 \\
\hline Croton ceanothifolius Baill. & Subshrub & $\mathrm{CR}$ & NY 870391 \\
\hline Croton celtidifolius Baill. & Tree & $\mathrm{F}$ & OUPR 1242 \\
\hline Croton fuscescens Spreng. & Subshrub & $\mathrm{CR}, \mathrm{F}$ & OUPR 20289 \\
\hline Croton lundianus (Didr.) Müll. Arg. & Subshrub & $\mathrm{CR}$ & OUPR 13619 \\
\hline Croton salutaris Casar. & Tree & $\mathrm{F}$ & OUPR 1281 \\
\hline Croton vulnerarius Baill. & Tree & $\mathrm{CR}, \mathrm{F}$ & OUPR 6092 \\
\hline Euphorbia chrysophylla (Klotzsch \& Garcke) Boiss. & Subshrub & $\mathrm{CR}$ & BHCB 602 \\
\hline Euphorbia hyssopifolia L. & Subshrub & $\mathrm{CR}$ & OUPR 1408 \\
\hline Euphorbia portulacoides subsp. collina (Phil.) Croizat & Subshrub & $\mathrm{CR}$ & BHCB 602 \\
\hline Manihot pilosa Pohl & Shrub & F & OUPR 18256 \\
\hline Microstachys daphnoides (Mart.) Müll. Arg. & Shrub & $\mathrm{CR}$ & OUPR 6930 \\
\hline Microstachys hispida (Mart.) Govaerts & Subshrub & $\mathrm{CR}$ & RB 277642 \\
\hline Sapium glandulosum (L.) Morong & Shrub & $\mathrm{F}$ & OUPR 1213 \\
\hline Sapium sellowianum (Müll.Arg.) Klotzsch ex Baill. & Tree & $\mathrm{F}$ & NY 528680 \\
\hline \multicolumn{4}{|l|}{ Fabaceae } \\
\hline Abarema brachystachya (DC.) Barneby \& J.W.Grimes & Tree & $\mathrm{CR}$ & OUPR 21252 \\
\hline Abarema langsdorffii (Benth.) Barneby \& J.W.Grimes & Tree & $\mathrm{CR}$ & OUPR 18931 \\
\hline Aeschynomene elegans Schltdl. \& Cham & Subshrub & $\mathrm{CR}$ & OUPR 13590 \\
\hline Anadenanthera colubrina (Vell.) Brenan & Tree & $\mathrm{F}$ & OUPR 20242 \\
\hline Anadenanthera peregrina (L.) Speg. & Tree & $\mathrm{F}$ & OUPR 21354 \\
\hline Ancistrotropis peduncularis (Kunth) A. Delgado & Liana & $\mathrm{CR}$ & OUPR 13587 \\
\hline Andira fraxinifolia Benth. & Tree & $\mathrm{F}$ & VIC 20033 \\
\hline Andira humilis Mart. ex Benth. & Shrub & $\mathrm{CR}$ & OUPR 21201 \\
\hline Andira surinamensis (Bondt) Splitg. ex Amshoff & Tree & $\mathrm{F}$ & RB 411625 \\
\hline Bauhinia forficata Link & Tree & $\mathrm{F}$ & OUPR 15889 \\
\hline Bauhinia fusconervis (Bong.) Steud. & Tree & $\mathrm{F}$ & RB 98287 \\
\hline Bauhinia longifolia (Bong.) Steud. & Tree & $\mathrm{F}$ & VIC 20875 \\
\hline Bauhinia ungulata var. cuiabensis (Bong.) Vaz & Shrub & $\mathrm{F}$ & OUPR 20590 \\
\hline Bauhinia sp & Shrub & $\mathrm{F}$ & OUPR 7403 \\
\hline Bionia bella Mart. ex Benth. & Liana & $\mathrm{F}$ & OUPR 1306 \\
\hline Bowdichia virgilioides Kunth & Tree & $\mathrm{F}$ & OUPR 20575 \\
\hline Cajanus cajan (L.) Huth & Shrub & AA & OUPR 12125 \\
\hline Calliandra parvifolia (Hook. \& Arn.) Speg. & Shrub & $\mathrm{F}$ & OUPR 19479 \\
\hline Calliandra surinamensis Benth. & Shrub & $\mathrm{F}$ & OUPR 7349 \\
\hline Calopogonium mucunoides Desv. & Subshrub & $\mathrm{CR}$ & OUPR 12131 \\
\hline Camptosema scarlatinum var. pohlianum (Benth.) Burkart & Liana & $\mathrm{CR}$ & OUPR 13608 \\
\hline Cassia ferruginea (Schrad.) Schrad ex DC. & Tree & $\mathrm{F}$ & OUPR 20585 \\
\hline Centrosema coriaceum Benth. & Liana & $\mathrm{CR}$ & OUPR 1407 \\
\hline Centrosema virginianum (L.) Benth. & Liana & $\mathrm{CR}$ & OUPR 12134 \\
\hline Chaetocalyx longiflora Benth. ex A.Gray & Liana & F & VIC 21000 \\
\hline Chamaecrista andromedea (Mart. ex Benth.) H.S.Irwin \& Barneby & Shrub & $\mathrm{CR}$ & OUPR 3489 \\
\hline Chamaecrista calycioide s(DC. ex Collad.) Greene & Subshrub & $\mathrm{CR}$ & VIC 28986 \\
\hline Chamaecrista dentata (Vogel) H.S.Irwin \& Barneby EN* & Shrub & $\mathrm{CR}$ & OUPR 1220 \\
\hline Chamaecrista desvauxii (Collad.) Killip & Subshrub & $\mathrm{CR}$ & OUPR 22195 \\
\hline Chamaecrista flexuosa (L.) Greene var. flexuosa & Shrub & $\mathrm{CR}$ & OUPR 15934 \\
\hline Chamaecrista hedysaroides (Vogel) H.S.Irwin \& Barneby & Shrub & $\mathrm{CR}, \mathrm{F}$ & OUPR 19290 \\
\hline Chamaecrista langsdorffii (Kunth ex Vogel) Britton ex Pittier & Shrub & $\mathrm{CR}$ & OUPR 1423 \\
\hline Chamaecrista mucronata (Spreng.) H.S.Irwin \& Barneby. & Shrub & $\mathrm{CR}$ & OUPR 1349 \\
\hline Chamaecrista multipennis (H.S.Irwin \& Barneby) H.S.Irwin \& Barneby & Shrub & $\mathrm{CR}$ & OUPR 18612 \\
\hline Chamaecrista nictitans (L.) Moench & Subshrub & $\mathrm{CR}$ & OUPR 16603 \\
\hline Chamaecrista ochnacea (Vogel) H.S.Irwin \& Barneby & Shrub & $\mathrm{CR}$ & OUPR 20197 \\
\hline Chamaecrista rotundata var. grandistipula (Vogel) H.S.Irwin \& Barneby & Shrub & $\mathrm{CR}$ & OUPR 18982 \\
\hline Chamaecrista rotundata var. rotundata (Vogel) H.S.Irwin \& Barneby & Shrub & $\mathrm{CR}$ & OUPR 18982 \\
\hline Chamaecrista rotundifolia var. rotundifolia (Pers.) Greene & Herb & $\mathrm{CR}$ & OUPR 18980 \\
\hline Chamaecrista trichopoda (Benth.) Britton \& Rose ex Britton \& Killip & Subshrub & $\mathrm{CR}$ & VIC 28986 \\
\hline Chamaecrista sp & Shrub & $\mathrm{CR}$ & OUPR 7400 \\
\hline
\end{tabular}


Table 1. Continued...

\begin{tabular}{|c|c|c|c|}
\hline Family / Species & Habit & Habitat & Voucher \\
\hline Clitoria falcata Lam. var. falcata & Liana & $\mathrm{F}$ & OUPR 18254 \\
\hline Clitoria rufescens (Benth.) Benth. & Liana & $\mathrm{CR}$ & OUPR 1433 \\
\hline Collaea speciosa (Loisel.) DC. & Subshrub & $\mathrm{CR}$ & OUPR 17741 \\
\hline Copaifera langsdorffii Desf. & Tree & $\mathrm{F}$ & RB 377703 \\
\hline Copaifera reticulata Ducke & Tree & $\mathrm{F}$ & OUPR 13761 \\
\hline Crotalaria breviflora DC. & Subshrub & $\mathrm{F}$ & OUPR 13501 \\
\hline Crotalaria grandiflora Benth. & Subshrub & $\mathrm{CR}$ & OUPR 2337 \\
\hline Crotalaria incana $\mathrm{L}$. & Subshrub & $\mathrm{CR}$ & OUPR 14408 \\
\hline Crotalaria lanceolata E.Mey. & Subshrub & $\mathrm{CR}$ & OUPR 14414 \\
\hline Crotalaria maypurensis Kunth & Subshrub & $\mathrm{CR}$ & NY 983419 \\
\hline Crotalaria micans Link & Subshrub & $\mathrm{CR}$ & OUPR 1279 \\
\hline Crotalaria nitens Kunth & Subshrub & $\mathrm{CR}$ & OUPR 13813 \\
\hline Crotalaria otoptera Benth. & Subshrub & $\mathrm{CR}$ & OUPR 5256 \\
\hline Crotalaria pallida Aiton & Subshrub & CR & FUNED 1275 \\
\hline Crotalaria paulina Schrank & Subshrub & $\mathrm{F}$ & OUPR 4817 \\
\hline Crotalaria pilosa Mill. & Subshrub & $\mathrm{CR}$ & VIC 24905 \\
\hline Crotalaria stipularia Desv. & Subshrub & $\mathrm{CR}$ & VIC 28478 \\
\hline Crotalaria velutina Benth. & Subshrub & $\mathrm{CR}$ & VIC 28479 \\
\hline Dalbergia acuta Benth. & Shrub & $\mathrm{CR}$ & RB 404382 \\
\hline Dalbergia brasiliensis Vogel & Tree & $\mathrm{F}$ & OUPR 21241 \\
\hline Dalbergia foliosa (Benth.) A.M.Carvalho & Shrub & $\mathrm{CR}$ & RB 404382 \\
\hline Dalbergia frutescens (Vell.) Britton & Shrub & $\mathrm{CR}, \mathrm{F}$ & OUPR 9091 \\
\hline Dalbergia nigra (Vell.) Allemão ex Benth. VU* & Tree & $\mathrm{CR}, \mathrm{F}$ & OUPR 8254 \\
\hline Dalbergia villosa (Benth.) Benth.var. villosa & Tree & $\mathrm{CR}, \mathrm{F}$ & OUPR 12140 \\
\hline Dalbergia violacea (Jacq.) Hoffmanns. & Tree & $\mathrm{F}$ & OUPR 6377 \\
\hline Dalbergia sp & Tree & $\mathrm{F}$ & VIC 21402 \\
\hline Desmodium adscendens (Sw.) DC. & Subshrub & $\mathrm{CR}, \mathrm{F}$ & OUPR 12127 \\
\hline Desmodium affine Schltdl. & Subshrub & $\mathrm{CR}$ & OUPR 12129 \\
\hline Desmodium barbatum (L.) Benth. & Subshrub & $\mathrm{CR}$ & OUPR 14418 \\
\hline Desmodium incanum DC. & Subshrub & $\mathrm{CR}$ & OUPR 14402 \\
\hline Desmodium subsericeum Malme & Subshrub & $\mathrm{CR}$ & ICN 138601 \\
\hline Desmodium uncinatum (Jacq.) DC. & Subshrub & $\mathrm{CR}, \mathrm{F}$ & OUPR 12130 \\
\hline Dioclea violacea Mart. ex Benth. & Liana & $\mathrm{F}$ & VIC 21026 \\
\hline Eriosema crinitum var. discolor Fortunato & Subshrub & $\mathrm{CR}$ & OUPR 19766 \\
\hline Erythrina speciosa Andrews & Tree & $\mathrm{CR}$ & OUPR 13711 \\
\hline Indigofera suffruticosa Mill. & Subshrub & $\mathrm{CR}$ & OUPR 12124 \\
\hline Inga barbata Benth. & Tree & CR & OUPR 18930 \\
\hline Inga cylindrica (Vell.) Mart. & Tree & $\mathrm{F}$ & OUPR 21340 \\
\hline Inga edulis Mart. & Tree & $\mathrm{F}$ & VIC 20961 \\
\hline Inga ingoides (Rich.) Willd. & Tree & $\mathrm{F}$ & OUPR 21339 \\
\hline Inga marginata Willd. & Tree & $\mathrm{F}$ & OUPR 20583 \\
\hline Inga schinifolia Benth. & Tree & $\mathrm{F}$ & VIC 20963 \\
\hline Inga sessilis (Vell.) Mart. & Tree & $\mathrm{F}$ & OUPR 1439 \\
\hline Inga vera subsp. affinis (DC.) T.D.Penn. & Tree & $\mathrm{F}$ & OUPR 20581 \\
\hline Inga vulpina Mart. ex Benth. & Tree & $\mathrm{CR}, \mathrm{F}$ & OUPR 9121 \\
\hline Inga $\mathrm{sp} 1$ & Tree & $\mathrm{F}$ & OUPR 7401 \\
\hline Inga $\mathrm{sp} 2$ & Tree & $\mathrm{F}$ & OUPR 9060 \\
\hline Leptospron adenanthum (G. Mey.) A.Delgado & Liana & $\mathrm{CR}$ & RB 255436 \\
\hline Machaerium aculeatum Raddi & Tree & $\mathrm{CR}, \mathrm{F}$ & OUPR 4234 \\
\hline Machaerium brasiliense Vogel LC ${ }^{* * * *}$ & Shrub & $\mathrm{CR}$ & OUPR 13611 \\
\hline Machaerium hirtum (Vell.) Stellfeld & Tree & $\mathrm{F}$ & OUPR 8259 \\
\hline Machaerium nyctitans (Vell.) Benth. & Tree & $\mathrm{F}$ & OUPR 20070 \\
\hline Machaerium oblongifolium Vogel & Liana & $\mathrm{F}$ & OUPR 13715 \\
\hline Machaerium reticulatum (Poir.) Pers. & Tree & $\mathrm{F}$ & OUPR 13712 \\
\hline Machaerium villosum Vogel VU $\mathrm{U}^{* * * * *}$ & Tree & $\mathrm{F}$ & OUPR 20071 \\
\hline Machaerium $\mathrm{sp}$ & Shrub & $\mathrm{F}$ & OUPR 8259 \\
\hline Macroptilium sabaraense (Hoehne) V.P.Barbosa & Liana & $\mathrm{CR}$ & VIC 29114 \\
\hline Melanoxylon brauna Schott VU*, ** & Tree & $\mathrm{F}$ & OUPR 7405 \\
\hline
\end{tabular}


MESSIAS, M.C.T.B. et al.

Table 1. Continued..

\begin{tabular}{|c|c|c|c|}
\hline Family / Species & Habit & Habitat & Voucher \\
\hline Mimosa aurivillus Mart. var. aurivillus & Shrub & CR & OUPR 19029 \\
\hline Mimosa aurivillus var. calothamnos (Benth.) Barneby & Shrub & $\mathrm{CR}$ & OUPR 1223 \\
\hline Mimosa bimucronata (DC.) Kuntze var. bimucronata & Shrub & $\mathrm{F}$ & OUPR 19477 \\
\hline Mimosa calodendron Mart. ex Benth. & Shrub & $\mathrm{CR}$ & OUPR 24799 \\
\hline Mimosa debilis Humb. \& Bonpl. ex Willd. var. debilis & Subshrub & $\mathrm{CR}$ & OUPR 12803 \\
\hline Mimosa diplotricha C.Wright ex Sauvalle var. diplotricha & Liana & CR & VIC 30737 \\
\hline Mimosa dolens Vell. var. dolens & Subshrub & $\mathrm{CR}$ & OUPR 18927 \\
\hline Mimosa montis-carasae Barneby EN*, ${ }^{* *}$ & Shrub & $\mathrm{CR}$ & OUPR 1452 \\
\hline Mimosa ourobrancoensis Burkart & Shrub & $\mathrm{CR}$ & OUPR 6385 \\
\hline Mimosa peduncularis Bong. ex Benth. & Shrub & $\mathrm{CR}$ & CEN 80702 \\
\hline Mimosa pigra $\mathrm{L}$. & Shrub & $\mathrm{CR}$ & VIC 28302 \\
\hline Mimosa pudica var. hispida Brenan & Subshrub & $\mathrm{CR}$ & VIC 30736 \\
\hline Mimosa scabrella Benth. & Tree & $\mathrm{CR}, \mathrm{F}$ & OUPR 12408 \\
\hline Mimosa sensitiva var. malitiosa (Mart.) Barneby & Liana & CR & VIC 30738 \\
\hline Ormosia friburgensis Taub. ex Glaz. & Tree & $\mathrm{F}$ & VIC 21045 \\
\hline Ormosia ruddiana Yakovlev & Tree & $\mathrm{F}$ & RB 419029 \\
\hline Parapiptadenia rigida (Benth.) Brenan & Tree & $\mathrm{F}$ & OUPR 1328 \\
\hline Peltophorum dubium (Spreng.) Taub. & Tree & $\mathrm{F}$ & NY 1093141 \\
\hline Periandra mediterranea (Vell.) Taub. & Shrub & $\mathrm{CR}$ & OUPR 14407 \\
\hline Piptadenia adiantoides (Spreng.) J.F.Macbr. & Shrub & $\mathrm{F}$ & OUPR 868 \\
\hline Piptadenia gonoacantha (Mart.) J.F.Macbr. & Tree & $\mathrm{F}$ & OUPR 20287 \\
\hline Piptadenia micracantha Benth. & Liana & $\mathrm{F}$ & OUPR 1450 \\
\hline Platypodium elegans Vogel & Tree & $\mathrm{F}$ & VIC 21048 \\
\hline Poiretia punctata (Willd.) Desv. & Liana & $\mathrm{F}$ & OUPR 19092 \\
\hline Pseudopiptadenia contorta (DC.) G.P. Lewis \& M.P. Lima & Tree & $\mathrm{F}$ & OUPR 20582 \\
\hline Pterocarpus rohrii Vahl & Tree & $\mathrm{F}$ & OUPR 15587 \\
\hline Rhynchosia minima (L.) DC. & Liana & $\mathrm{CR}$ & OUPR 19110 \\
\hline Rhynchosia reticulata (Sw.) DC. & Liana & $\mathrm{CR}$ & OUPR 19024 \\
\hline Schnella macrostachya Raddi & Liana & $\mathrm{CR}$ & VIC 20874 \\
\hline Senegalia martiusiana (Steud.) Seigler \& Ebinger & Liana & $\mathrm{F}$ & VIC 20930 \\
\hline Senegalia nitidifolia (Speg.) Seigler \& Ebinger & Shrub & $\mathrm{F}$ & RB 421786 \\
\hline Senegalia riparia (Kunth) Britton \& Rose ex Britton \& Killip & Liana & $\mathrm{F}$ & VIC 20935 \\
\hline Senna bicapsularis (L.) Roxb. var. bicapsularis & Shrub & CR & OUPR 18666 \\
\hline Senna macranthera (DC. ex Collad.) H.S.Irwin \& Barneby & Tree & $\mathrm{F}$ & OUPR 1346 \\
\hline Senna macranthera var. nervosa (Vogel) H.S.Irwin \& Barneby & Tree & $\mathrm{F}$ & OUPR 1347 \\
\hline Senna multijuga var. lindleyana (Gardner) H.S.Irwin \& Barneby & Tree & $\mathrm{F}$ & OUPR 20587 \\
\hline Senna neglecta var. oligophylla (Benth.) H.S.Irwin \& Barneby & Tree & CR & OUPR 20197 \\
\hline Senna pendula var. glabrata (Vogel) H.S.Irwin \& Barneby & Shrub & $\mathrm{CR}, \mathrm{F}$ & OUPR 8258 \\
\hline Senna pneumatica H.S.Irwin \& Barneby & Shrub & $\mathrm{F}$ & OUPR 20589 \\
\hline Senna reniformis (G.Don) H.S.Irwin \& Barneby & Shrub & $\mathrm{CR}, \mathrm{F}$ & OUPR 1305 \\
\hline Sesbania virgata (Cav.) Pers. & Shrub & CR & OUPR 19022 \\
\hline Spartium junceum L. & Shrub & AA & OUPR 13710 \\
\hline Stryphnodendron adstringens (Mart.) Coville & Tree & $\mathrm{F}$ & OUPR 7443 \\
\hline Stryphnodendron polyphyllum Mart. & Tree & $\mathrm{F}$ & OUPR 21204 \\
\hline Stylosanthes gracilis Kunth & Subshrub & $\mathrm{CR}$ & OUPR 19922 \\
\hline Stylosanthes guianensis var. guianensis (Aubl.) Sw. & Subshrub & $\mathrm{CR}$ & MO 3210340 \\
\hline Stylosanthes guianensis var. pauciflora M.B.Ferreira \& Sousa Costa & Subshrub & $\mathrm{CR}$ & NY 623171 \\
\hline Stylosanthes macrocephala M.B.Ferreira \& Sousa Costa & Subshrub & $\mathrm{CR}$ & OUPR 12126 \\
\hline Stylosanthes montevidensis Vogel & Subshrub & $\mathrm{CR}$ & OUPR 12126 \\
\hline Stylosanthes scabra Vogel & Subshrub & $\mathrm{CR}$ & RB 190484 \\
\hline Stylosanthes viscosa (L.) Sw. & Subshrub & $\mathrm{CR}$ & OUPR 13599 \\
\hline Swartzia hilaireana Mansano \& Torke & Tree & $\mathrm{F}$ & OUPR 21248 \\
\hline Swartzia oblata R.S.Cowan & Shrub & $\mathrm{CR}$ & OUPR 19025 \\
\hline Tachigali friburgensis (Harms) L.G.Silva \& H.C.Lima & Tree & $\mathrm{F}$ & OUPR 20588 \\
\hline Tachigali rugosa (Mart. ex Benth.) Zarucchi \& Pipoly & Tree & $\mathrm{F}$ & OUPR 7449 \\
\hline Trifolium repens $\mathrm{L}$. & Subshrub & $\mathrm{F}$ & OUPR 13754 \\
\hline Zornia curvata Mohlenbr. & Subshrub & $\mathrm{CR}$ & OUPR 27805 \\
\hline Zornia hebecarpa Mohlenbr. & Subshrub & $\mathrm{CR}$ & OUPR 27798 \\
\hline
\end{tabular}


Table 1. Continued...

\begin{tabular}{|c|c|c|c|}
\hline Family / Species & Habit & Habitat & Voucher \\
\hline Zornia reticulata $\mathrm{Sm}$. & Subshrub & $\mathrm{CR}$ & OUPR 8256 \\
\hline \multicolumn{4}{|l|}{ Gentianaceae } \\
\hline Calolisianthus amplissimus (Mart.) Gilg & Subshrub & $\mathrm{CR}$ & UPCB 38616 \\
\hline Calolisianthus pedunculatus (Cham. \& Schltdl.) Gilg & Subshrub & $\mathrm{CR}$ & OUPR 4564 \\
\hline Calolisianthus pendulus (Mart.) Gilg & Subshrub & CR & OUPR 15385 \\
\hline Calolisianthus pulcherrimus (Mart.) Gilg & Subshrub & CR & OUPR 1375 \\
\hline Calolisianthus speciosus (Cham. \& Schltdl.) Gilg & Subshrub & $\mathrm{CR}$ & VIC 8272 \\
\hline Deianira damazioi E.F.Guimar. & Subshrub & CR & RB 471530 \\
\hline Deianira nervosa Cham. \& Schltdl. & Subshrub & CR & OUPR 9094 \\
\hline Helia alpestris (Mart.) Kuntze & Subshrub & CR & OUPR 16387 \\
\hline Irlbachia elegans Mart. & Subshrub & $\mathrm{CR}$ & OUPR 20470 \\
\hline \multicolumn{4}{|l|}{ Gesneriaceae } \\
\hline Anetanthus gracilis Hiern VU* & Subshrub & CR & OUPR 13296 \\
\hline Nematanthus dichrus (Spreng.) Wiehler & Subshrub & $\mathrm{CR}, \mathrm{F}$ & OUPR 1215 \\
\hline Paliavana sericiflora Benth. & Subshrub & CR & OUPR 1350 \\
\hline Sinningia allagophylla (Mart.) Wiehler & Subshrub & $\mathrm{CR}$ & OUPR 1271 \\
\hline Sinningia elatior (Kunth) Chautems & Subshrub & $\mathrm{CR}$ & OUPR 17707 \\
\hline Sinningia magnifica (Otto \& A.Dietr.) Wiehler & Subshrub & CR & OUPR 13427 \\
\hline Sinningia tuberosa (Mart.) H.E.Moore VU*, & Subshrub & $\mathrm{CR}$ & OUPR 16437 \\
\hline \multicolumn{4}{|l|}{ Griseliniaceae } \\
\hline Griselinia ruscifolia (Clos) Taub. & Liana & CR & OUPR 3307 \\
\hline \multicolumn{4}{|l|}{ Humiriaceae } \\
\hline Humiriastrum glaziovii (Urb.) Cuatrec. & Tree & $\mathrm{F}$ & RB 277735 \\
\hline Sacoglottis dentata (Casar.) Urb. & Tree & $\mathrm{F}$ & OUPR 3563 \\
\hline \multicolumn{4}{|l|}{ Hydrangeaceae } \\
\hline Hydrangea macrophylla (Thunb.) Ser. & Subshrub & AA & OUPR 21501 \\
\hline \multicolumn{4}{|l|}{ Hypericaceae } \\
\hline Hypericum brasiliense Choisy & Subshrub & CR & RB 98245 \\
\hline Hypericum denudatum A.St.-Hil. & Subshrub & CR & RB 148714 \\
\hline Hypericum polyanthemum Klotzsch ex Reichardt & Subshrub & $\mathrm{CR}, \mathrm{F}$ & OUPR 1876 \\
\hline Hypericum rigidum A.St.-Hil. & Subshrub & CR & BHCB 8278 \\
\hline Vismia brasiliensis Choisy & Shrub & CR & OUPR 12795 \\
\hline Vismia magnoliifolia Cham. \& Schltdl. & Shrub & $\mathrm{CR}, \mathrm{F}$ & OUPR 1216 \\
\hline Vismia micrantha A.St.-Hil. & Shrub & $\mathrm{CR}$ & OUPR 12799 \\
\hline Vismia parviflora Cham. \& Schltdl. & Shrub & $\mathrm{CR}, \mathrm{F}$ & OUPR 1284 \\
\hline \multicolumn{4}{|l|}{ Hypoxidaceae } \\
\hline Hypoxis decumbens $\mathrm{L}$. & Herb & $\mathrm{CR}$ & OUPR 17505 \\
\hline \multicolumn{4}{|l|}{ Iridaceae } \\
\hline Crocosmia crocosmiiflora (Lemoine ex Morren) N.E.Br. & Herb & CR & OUPR 1348 \\
\hline Cypella caerulea(Ker Gawl.) Seub. ex Hook. f. & Herb & CR & OUPR 15601 \\
\hline Cypella sp & Herb & CR & OUPR 13554 \\
\hline Dietes bicolor Sweet ex Klatt & Herb & $\mathrm{CR}$ & OUPR 19307 \\
\hline Neomarica caerulea (Ker Gawl.) Sprague & Herb & CR & OUPR 13546 \\
\hline Neomarica glauca (Seub. ex Klatt) Sprague & Herb & CR & OUPR 13545 \\
\hline Sisyrinchium itabiritense Ravenna & Herb & $\mathrm{CR}$ & NY 910565 \\
\hline Sisyrinchium minutiflorum Klatt & Herb & CR & OUPR 11513 \\
\hline Sisyrinchium vaginatum Spreng. & Herb & CR & OUPR 9096 \\
\hline Sisyrinchium sp & Herb & $\mathrm{CR}$ & OUPR 23282 \\
\hline Trimezia juncifolia (Klatt) Benth. \& Hook. & Herb & CR & OUPR 10774 \\
\hline Trimezia rupestris Ravenna & Herb & CR & MBML 7050 \\
\hline \multicolumn{4}{|l|}{ Juncaceae } \\
\hline Juncus microcephalus Kunth & Herb & $\mathrm{CR}$ & OUPR 15950 \\
\hline Juncus tenuis Willd. & Herb & $\mathrm{CR}$ & NY 402417 \\
\hline Juncus sp1 & Herb & $\mathrm{CR}$ & OUPR 22510 \\
\hline Juncus sp2 & Herb & $\mathrm{CR}$ & OUPR 22511 \\
\hline \multicolumn{4}{|l|}{ Lacistemataceae } \\
\hline Lacistema pubescens Mart. & Shrub & $\mathrm{F}$ & OUPR 10769 \\
\hline Lamiaceae & & & \\
\hline
\end{tabular}


MESSIAS, M.C.T.B. et al.

Table 1. Continued..

\begin{tabular}{|c|c|c|c|}
\hline Family / Species & Habit & Habitat & Voucher \\
\hline Aegiphila integrifolia (Jacq.) Moldenke & Shrub & $\mathrm{F}$ & OUPR 7295 \\
\hline Aegiphila obducta Vell. & Shrub & $\mathrm{CR}$ & OUPR 12407 \\
\hline Aegiphila verticillata Vell. & Shrub & $\mathrm{F}$ & RB 183422 \\
\hline Cantinoa racemulosa (Mart. ex Benth.) Harley \& J.F.B.Pastore & Subshrub & $\mathrm{CR}$ & NY 500959 \\
\hline Eriope macrostachya Mart. ex Benth. & Shrub & $\mathrm{CR}$ & OUPR 1293 \\
\hline Hyptidendron asperrimum (Spreng.) Harley & Tree & $\mathrm{CR}, \mathrm{F}$ & OUPR 5609 \\
\hline Hyptis homalophylla Pohl ex Benth. & Subshrub & $\mathrm{CR}$ & OUPR 16291 \\
\hline Hyptis monticola Mart. ex Benth. & Subshrub & $\mathrm{CR}$ & OUPR 17860 \\
\hline Hyptis pusilla (Pohl) Harley \& J.F.B.Pastore & Subshrub & $\mathrm{CR}$ & UB 172265 \\
\hline Hyptis radicans (Pohl) Harley \& J.F.B.Pastore & Subshrub & $\mathrm{CR}$ & OUPR 12804 \\
\hline Hyptis rotundifolia Benth. & Subshrub & $\mathrm{CR}$ & OUPR 17887 \\
\hline Leonurus japonicus Houtt. & Subshrub & $\mathrm{CR}$ & OUPR 4233 \\
\hline Marsypianthes chamaedrys (Vahl) Kuntze & Subshrub & $\mathrm{CR}$ & OUPR 7347 \\
\hline Mesosphaerum pectinatum (L.) Kuntze & Subshrub & $\mathrm{CR}$ & UEFS 150032 \\
\hline Mesosphaerum sidifolium (L'Hérit.) Harley \& J.F.B.Pastore & Subshrub & $\mathrm{CR}$ & UB 111148 \\
\hline Oocephalus oppositiflorus (Schrank) Harley \& J.F.B.Pastore & Subshrub & $\mathrm{CR}$ & OUPR 17830 \\
\hline Vitex polygama Cham. & Tree & $\mathrm{CR}, \mathrm{F}$ & OUPR 1376 \\
\hline Vitex rufescens A.Juss. & Tree & $\mathrm{F}$ & UB 161215 \\
\hline Vitex sellowiana Cham. & Shrub & $\mathrm{F}$ & OUPR 6422 \\
\hline \multicolumn{4}{|l|}{ Lauraceae } \\
\hline Cassytha filiformis L. & Liana & $\mathrm{F}$ & OUPR 20257 \\
\hline Cinnamomum erythropus (Nees \& Mart.) Kosterm. EN*, ,* & Tree & F & RB 418224 \\
\hline Cinnamomum quadrangulum Kosterm. VU,*** & Shrub & $\mathrm{CR}$ & OUPR 22531 \\
\hline Cinnamoтит sp. & Tree & $\mathrm{F}$ & RB 48737 \\
\hline Nectandra nitidula Nees \& Mart. & Tree & $\mathrm{CR}, \mathrm{F}$ & OUPR 7375 \\
\hline Nectandra oppositifolia Nees \& Mart. & Tree & $\mathrm{F}$ & OUPR 25200 \\
\hline Ocotea densiflora (Meisn.) Mez & Tree & $\mathrm{F}$ & OUPR 18144 \\
\hline Ocotea diospyrifolia (Meisn.) Mez & Tree & $\mathrm{F}$ & OUPR 21331 \\
\hline Ocotea dispersa (Nees \& Mart.) Mez & Tree & $\mathrm{F}$ & OUPR 15550 \\
\hline Ocotea divaricata (Nees) Mez & Shrub & $\mathrm{F}$ & OUPR 1489 \\
\hline Ocotea felix Coe-Teix. $\mathrm{CR}^{*}, \mathrm{EN}^{* *}$ & Shrub & $\mathrm{F}$ & OUPR 8331 \\
\hline Ocotea hypoglauca (Nees \& Mart.) Mez & Shrub & $\mathrm{CR}, \mathrm{F}$ & OUPR 18154 \\
\hline Ocotea lancifolia (Schott) Mez & Tree & $\mathrm{F}$ & RB 324873 \\
\hline Ocotea laxa (Nees) Mez & Tree & $\mathrm{F}$ & OUPR 6827 \\
\hline Ocotea nutans (Nees) Mez & Tree & F & RB 48725 \\
\hline Ocotea percoriacea Kosterm. EN* & Tree & $\mathrm{CR}, \mathrm{F}$ & OUPR 18154 \\
\hline Ocotea pomaderroides (Meisn.) Mez EN* & Shrub & $\mathrm{F}$ & OUPR 19284 \\
\hline Ocotea puberula $($ Rich.) Nees & Tree & $\mathrm{F}$ & OUPR 21697 \\
\hline Ocotea sassafras (Meisn.) Mez. & Tree & $\mathrm{F}$ & OUPR 18195 \\
\hline Ocotea semicompleta (Nees \& Mart.) Mez & Tree & F & MO 2158206 \\
\hline Ocotea spixiana (Nees) Mez & Tree & $\mathrm{F}$ & OUPR 1390 \\
\hline Ocotea tabacifolia (Meisn.) Rohwer EN** & Shrub & F & OUPR 18144 \\
\hline Ocotea tristis (Nees \& C. Mart.) Mez & Shrub & $\mathrm{CR}$ & OUPR 1394 \\
\hline Ocotea vaccinioides (Meisn.) Mez & Shrub & $\mathrm{CR}$ & OUPR 18270 \\
\hline Ocotea $\mathrm{sp} 1$ & Shrub & $\mathrm{F}$ & OUPR 9072 \\
\hline Ocotea $\mathrm{sp} 2$ & Shrub & $\mathrm{F}$ & OUPR 6801 \\
\hline Persea americana Mill. & Tree & AA & OUPR 19302 \\
\hline Persea fulva L.E.Kopp & Tree & $\mathrm{F}$ & MO 1899923 \\
\hline Persea pedunculosa Meisn. EN** & Tree & $\mathrm{F}$ & RB 48653 \\
\hline \multicolumn{4}{|l|}{ Lecythidaceae } \\
\hline Cariniana estrellensis (Raddi) Kuntze & Tree & $\mathrm{F}$ & OUPR 140 \\
\hline \multicolumn{4}{|l|}{ Lentibulariaceae } \\
\hline Genlisea aurea A.St.-Hil. & Herb & $\mathrm{CR}$ & OUPR 3544 \\
\hline Genlisea repens Benj. & Herb & $\mathrm{CR}$ & OUPR 22811 \\
\hline Genlisea violacea A.St.-Hil. & Herb & $\mathrm{CR}$ & VIC 19479 \\
\hline Utricularia amethystina Salzm. ex A.St.-Hil. \& Girard & Herb & $\mathrm{CR}$ & OUPR 22812 \\
\hline \multicolumn{4}{|l|}{ Loganiaceae } \\
\hline Spigelia linarioides A.DC. & Subshrub & $\mathrm{CR}$ & RB 111041 \\
\hline
\end{tabular}


Table 1. Continued...

\begin{tabular}{|c|c|c|c|}
\hline Family / Species & Habit & Habitat & Voucher \\
\hline Spigelia spartioides Cham. & Subshrub & CR & OUPR 13473 \\
\hline \multicolumn{4}{|l|}{ Loranthaceae } \\
\hline Phthirusa sp & Shrub & $\mathrm{F}$ & BHCB 41488 \\
\hline Psittacanthus dichrous Mart. & Shrub & $\mathrm{F}$ & OUPR 1371 \\
\hline Struthanthus concinnus (Mart.) Mart. & Shrub & $\mathrm{F}$ & OUPR 1224 \\
\hline Struthanthus salicifolius Mart. & Shrub & $\mathrm{F}$ & OUPR 6770 \\
\hline \multicolumn{4}{|l|}{ Lythraceae } \\
\hline Cuphea carthagenensis (Jacq.) J.Macbr. & Subshrub & CR & OUPR 12579 \\
\hline Cuphea ingrata Cham. \& Schltdl. & Shrub & $\mathrm{CR}$ & OUPR 1227 \\
\hline Cuphea thymoides Cham. \& Schltdl. & Subshrub & $\mathrm{CR}$ & RB 74307 \\
\hline Diplusodon buxifolius (Cham. \& Schltdl.) A.DC. & Shrub & $\mathrm{CR}$ & OUPR 8336 \\
\hline Diplusodon hirsutus (Cham. \& Schltdl.) A.DC. & Shrub & $\mathrm{CR}$ & OUPR 1330 \\
\hline Diplusodon microphyllus Pohl & Subshrub & CR & OUPR 6823 \\
\hline Diplusodon virgatus Pohl & Subshrub & $\mathrm{CR}$ & OUPR 6824 \\
\hline Lafoensia pacari A.St.-Hil. & Shrub & $\mathrm{CR}$ & OUPR 16274 \\
\hline \multicolumn{4}{|l|}{ Magnoliaceae } \\
\hline Magnolia ovata (A.St.-Hil.) Spreng. & Tree & $\mathrm{CR}, \mathrm{F}$ & OUPR 5399 \\
\hline \multicolumn{4}{|l|}{ Malpighiaceae } \\
\hline Banisteriopsis angustifolia (A.Juss.) B.Gates & Subshrub & $\mathrm{CR}$ & OUPR 7788 \\
\hline Banisteriopsis campestris (A.Juss.) Little & Liana & CR & OUPR 7789 \\
\hline Banisteriopsis gardneriana (A.Juss.) W.R.Anderson \& B.Gates & Liana & $\mathrm{F}$ & OUPR 1459 \\
\hline Byrsonima clausseniana A.Juss. & Liana & $\mathrm{CR}, \mathrm{F}$ & OUPR 1228 \\
\hline Byrsonima intermedia A.Juss. & Shrub & $\mathrm{CR}$ & OUPR 1277 \\
\hline Byrsonima ligustrifolia A.Juss. & Tree & $\mathrm{F}$ & OUPR 20264 \\
\hline Byrsonima spinensis W.R.Anderson & Tree & $\mathrm{F}$ & SP 253566 \\
\hline Byrsonima variabilis A.Juss. & Shrub & CR & OUPR 12584 \\
\hline Heteropterys byrsonimifolia A.Juss. & Shrub & CR & SP 253567 \\
\hline Heteropterys intermedia (A.Juss.) Griseb. & Shrub & CR & NY 909452 \\
\hline Heteropterys umbellata A.Juss. & Shrub & $\mathrm{CR}$ & OUPR 1278 \\
\hline Heteropterys sp1 & Shrub & $\mathrm{F}$ & OUPR 3664 \\
\hline Mascagnia bierosa (A.Juss.) W.R.Anderson & Liana & $\mathrm{F}$ & NY 1131507 \\
\hline Peixotoa tomentosa A.Juss. & Subshrub & $\mathrm{CR}$ & OUPR 1369 \\
\hline Peixotoa $\mathrm{sp}$ & Shrub & $\mathrm{CR}$ & OUPR 9068 \\
\hline Tetrapterys microphylla (A.Juss.) Nied. & Subshrub & CR & RB 277643 \\
\hline Tetrapterys sp1 & Liana & $\mathrm{F}$ & OUPR 1460 \\
\hline Tetrapterys sp2 & Subshrub & CR & RB 277643 \\
\hline \multicolumn{4}{|l|}{ Malvaceae } \\
\hline Abutilon ibarrense Kunth & Shrub & $\mathrm{CR}$ & MO 3038566 \\
\hline Abutilon inaequilaterum A.St.-Hil. & Shrub & $\mathrm{CR}$ & OUPR 4854 \\
\hline Abutilon peltatum K.Schum. & Shrub & $\mathrm{F}$ & OUPR 4849 \\
\hline Byttneria scabra L. & Subshrub & $\mathrm{CR}$ & OUPR 21420 \\
\hline Ceiba sp & Tree & $\mathrm{F}$ & OUPR 7432 \\
\hline Krapovickasia macrodon (A.DC.) Fryxell & Subshrub & $\mathrm{CR}$ & OUPR 1232 \\
\hline Luehea divaricata Mart. \& Zucc. & Tree & $\mathrm{F}$ & OUPR 9073 \\
\hline Luehea paniculata Mart. \& Zucc. & Tree & $\mathrm{CR}, \mathrm{F}$ & OUPR 23176 \\
\hline Melochia pilosa (Mill.) Fawc. \& Rendle & Subshrub & $\mathrm{AA}, \mathrm{CR}$ & OUPR 24560 \\
\hline Pavonia communis A.St.-Hil. & Subshrub & CR & NY 407051 \\
\hline Pavonia montana Garcke ex Gürke & Shrub & $\mathrm{CR}$ & OUPR 4819 \\
\hline Pavonia sagittata A.St.-Hil. & Subshrub & $\mathrm{CR}$ & OUPR 4931 \\
\hline Pavonia schiedeana Steud. & Subshrub & $\mathrm{CR}$ & OUPR 1428 \\
\hline Pavonia schrankii Spreng. & Subshrub & CR & UEC 177669 \\
\hline Pavonia viscosa A.St.-Hil. & Shrub & $\mathrm{CR}, \mathrm{F}$ & OUPR 6796 \\
\hline Peltaea acutifolia (Gürke) Krapov. \& Cristóbal & Subshrub & CR & NY 00942549 \\
\hline Peltaea obsita (Mart. ex Colla) Krapov. \& Cristóbal & Subshrub & $\mathrm{CR}$ & NY 942549 \\
\hline Peltaea polymorpha (A.St.-Hil.) Krapov. \& Cristóbal & Shrub & $\mathrm{F}$ & OUPR 1405 \\
\hline Sida acuta Burm.f. & Subshrub & $\mathrm{CR}$ & NY 942698 \\
\hline Sida cordifolia L. & Subshrub & $\mathrm{F}$ & RB 399018 \\
\hline Sida glaziovii K.Schum. & Subshrub & $\mathrm{CR}$ & RB 398966 \\
\hline
\end{tabular}


Table 1. Continued..

\begin{tabular}{|c|c|c|c|}
\hline Family / Species & Habit & Habitat & Voucher \\
\hline Sida linifolia Cav. & Subshrub & $\mathrm{CR}$ & OUPR 19282 \\
\hline Sida rhombifolia $\mathrm{L}$. & Subshrub & $\mathrm{CR}$ & OUPR 1231 \\
\hline Sida spinosa $\mathrm{L}$. & Subshrub & $\mathrm{CR}$ & OUPR 13585 \\
\hline Sida tuberculata R.E.Fr. & Subshrub & $\mathrm{CR}$ & RB 342260 \\
\hline Sida viarum A.St.-Hil. & Subshrub & $\mathrm{CR}$ & SPF 74422 \\
\hline Triumfetta rhomboidea Jacq. & Subshrub & $\mathrm{CR}$ & OUPR 12576 \\
\hline Triumfetta semitriloba Jacq. & Subshrub & $\mathrm{CR}$ & NY 996627 \\
\hline Waltheria indica $\mathrm{L}$. & Subshrub & $\mathrm{CR}$ & OUPR 13618 \\
\hline Wissadula periplocifolia (L.) C.Presl. ex Thwaites & Subshrub & $\mathrm{CR}$ & OUPR 5007 \\
\hline \multicolumn{4}{|l|}{ Marantaceae } \\
\hline Goeppertia ackermannii (Körn.) Borchs. \& S.Suárez & Herb & $\mathrm{F}$ & OUPR 14075 \\
\hline \multicolumn{4}{|l|}{ Mayacaceae } \\
\hline Mayaca sellowiana Kunth & Herb & $\mathrm{CR}$ & OUPR 7585 \\
\hline \multicolumn{4}{|l|}{ Melastomataceae } \\
\hline Acisanthera variabilis (Naud.) Triana & Subshrub & $\mathrm{CR}$ & NY 2137296 \\
\hline Behuria glutinosa Cogn. & Shrub & $\mathrm{CR}$ & OUPR 20229 \\
\hline Cambessedesia corymbosa Mart. \& Schrank ex DC. & Subshrub & $\mathrm{CR}$ & UB 19511 \\
\hline Cambessedesia espora (A.St.-Hil. ex Bonpl.) DC. & Subshrub & $\mathrm{CR}$ & OUPR 23542 \\
\hline Cambessedesia fasciculata (Spreng.) Fidanza \& A.B. Martins & Subshrub & $\mathrm{CR}$ & NY 1549811 \\
\hline Cambessedesia hilariana (Kunth.) DC. & Subshrub & CR & OUPR 1292 \\
\hline Chaetostoma armatum (Spreng.) Cogn. & Subshrub & $\mathrm{CR}$ & RB 515229 \\
\hline Clidemia capitellata (Bonpl.) D.Don & Shrub & $\mathrm{CR}$ & BHCB 68080 \\
\hline Clidemia urceolata DC. & Shrub & $\mathrm{CR}$ & RB 515524 \\
\hline Comolia sertularia Triana & Shrub & $\mathrm{CR}$ & OUPR 21337 \\
\hline Eriocnema acaulis (Cham.) Triana EN** & Subshrub & $\mathrm{F}$ & UEC 104907 \\
\hline Eriocnema fulva Naudin $\mathrm{VU}^{*, * *}$ & Subshrub & $\mathrm{CR}$ & OUPR 5819 \\
\hline Fritzschia anisostemon Cham. VU* & Subshrub & $\mathrm{CR}$ & OUPR 20231 \\
\hline Fritzschia erecta Cham. & Subshrub & $\mathrm{CR}$ & OUPR 8419 \\
\hline Lavoisiera alba Mart. \& Schrank ex DC. & Subshrub & $\mathrm{CR}$ & HUFU 24943 \\
\hline Lavoisiera caparaoensis Schwacke ex Schwacke \& Cogn. & Subshrub & $\mathrm{CR}$ & BHCB 67488 \\
\hline Lavoisiera imbricata DC. & Subshrub & $\mathrm{CR}$ & OUPR 7382 \\
\hline Lavoisiera pulcherrima DC. & Shrub & $\mathrm{CR}$ & OUPR 1440 \\
\hline Leandra aurea (Cham.) Cogn. & Shrub & $\mathrm{F}$ & OUPR 5160 \\
\hline Leandra australis (Cham.) Cogn. & Shrub & $\mathrm{F}$ & OUPR 1420 \\
\hline Leandra cancellata Cogn. & Shrub & CR & $\begin{array}{c}\text { UPCB (Reginato, } \mathrm{M} . \\
1175)\end{array}$ \\
\hline Leandra carassana (DC.) Cogn. & Shrub & $\mathrm{F}$ & OUPR 515473 \\
\hline Leandra coriacea Cogn. & Subshrub & $\mathrm{CR}$ & HUFU 57647 \\
\hline Leandra dendroides (Naudin) Cogn. & Subshrub & $\mathrm{F}$ & RB 515390 \\
\hline Leandra erostrata (DC.) Cogn. & Subshrub & CR & RB 515387 \\
\hline Leandra fluminensis Cogn. & Subshrub & $\mathrm{F}$ & RB 515378 \\
\hline Leandra foveolata (DC.) Cogn. & Subshrub & $\mathrm{CR}$ & HUFU 57652 \\
\hline Leandra fragilis Cogn. & Shrub & $\mathrm{CR}$ & OUPR 20218 \\
\hline Leandra glabrata Cogn. & Shrub & $\mathrm{CR}$ & RB 515472 \\
\hline Leandra lacunosa Cogn. & Shrub & $\mathrm{CR}$ & RB 515478 \\
\hline Leandra lutea Cogn. & Shrub & $\mathrm{F}$ & NY 2099130 \\
\hline Leandra melastomoides Raddi & Shrub & $\mathrm{CR}, \mathrm{F}$ & OUPR 5831 \\
\hline Leandra polychaeta Cogn. & Shrub & $\mathrm{CR}$ & $\begin{array}{c}\text { UPCB (Reginato, M. } \\
1173 \text { ) }\end{array}$ \\
\hline Leandra purpurascens (DC.) Cogn. & Shrub & F & NY 02098871 \\
\hline Leandra quinquedentata (DC.) Cogn. & Shrub & $\mathrm{CR}$ & RB 515474 \\
\hline Leandra sulfurea Cogn. & Shrub & $\mathrm{CR}$ & RB 149572 \\
\hline Leandra sp1 & Shrub & $\mathrm{F}$ & OUPR 1238 \\
\hline Leandra sp2 & Shrub & $\mathrm{F}$ & OUPR 1419 \\
\hline Leandra sp3 & Subshrub & $\mathrm{CR}$ & OUPR 17297 \\
\hline Leandra sp4 & Subshrub & $\mathrm{CR}$ & OUPR 5833 \\
\hline Leandra sp5 & Shrub & $\mathrm{F}$ & OUPR 19285 \\
\hline Leandra sp6 & Shrub & $\mathrm{F}$ & OUPR 20164 \\
\hline
\end{tabular}


Table 1. Continued...

\begin{tabular}{|c|c|c|c|}
\hline Family / Species & Habit & Habitat & Voucher \\
\hline Marcetia cordigera DC. & Subshrub & $\mathrm{CR}$ & OUPR 5756 \\
\hline Marcetia taxifolia (A.St.-Hil.) DC. & Subshrub & $\mathrm{CR}$ & OUPR 1386 \\
\hline Miconia albicans (Sw.) Triana & Subshrub & $\mathrm{CR}$ & OUPR 6577 \\
\hline Miconia brunnea DC. & Tree & $\mathrm{F}$ & UEC 150710 \\
\hline Miconia calvescens Schrank \& Mart. ex DC. & Shrub & $\mathrm{F}$ & OUPR 1421 \\
\hline Miconia chartacea Triana & Tree & $\mathrm{F}$ & OUPR 1949 \\
\hline Miconia corallina Spring. & Shrub & $\mathrm{F}$ & OUPR 1233 \\
\hline Miconia cubatanensis Hoehne & Shrub & $\mathrm{F}$ & UPCB 71895 \\
\hline Miconia cyathanthera Triana & Shrub & $\mathrm{F}$ & OUPR 3705 \\
\hline Miconia discolor DC. & Tree & $\mathrm{F}$ & OUPR 19079 \\
\hline Miconia flammea Casar. & Shrub & $\mathrm{F}$ & RB 469741 \\
\hline Miconia ibaguensis (Bonpl.) Triana & Shrub & $\mathrm{F}$ & RB 469741 \\
\hline Miconia inconspicua Miq. & Tree & $\mathrm{F}$ & UPCB 71896 \\
\hline Miconia ligustroides (DC.) Naudin & Shrub & $\mathrm{F}$ & OUPR 1417 \\
\hline Miconia macrophylla (Pav. ex D. Don) Triana & Shrub & $\mathrm{F}$ & OUPR 1263 \\
\hline Miconia minutiflora (Bonpl.) DC. & Shrub & $\mathrm{F}$ & OUPR 4814 \\
\hline Miconia paniculata (DC.) Naudin & Shrub & $\mathrm{F}$ & NY 1738436 \\
\hline Miconia pennipilis Cogn. & Shrub & $\mathrm{CR}$ & OUPR 6566 \\
\hline Miconia rimalis Naudin. & Shrub & $\mathrm{F}$ & OUPR 1975 \\
\hline Miconia sellowiana Naudin & Shrub & $\mathrm{F}$ & RB 358485 \\
\hline Miconia shepherdii R.Goldenb. \& Reginato & Tree & $\mathrm{F}$ & RB 555987 \\
\hline Miconia stelligera Cogn. & Shrub & $\mathrm{F}$ & OUPR 6581 \\
\hline Miconia tentaculifera Naudin & Shrub & $\mathrm{F}$ & OUPR 1366 \\
\hline Miconia theizans (Bonpl.) Cogn. & Shrub & $\mathrm{CR}, \mathrm{F}$ & OUPR 21330 \\
\hline Miconia trianae Cogn. & Shrub & $\mathrm{CR}$ & OUPR 1234 \\
\hline Miconia willdenowii Klotzsch ex Naudin & Shrub & $\mathrm{F}$ & SPF203103 \\
\hline Miconia sp1 & Shrub & $\mathrm{F}$ & OUPR 1422 \\
\hline Miconia sp2 & Shrub & $\mathrm{CR}$ & OUPR 13614 \\
\hline Miconia sp3 & Shrub & $\mathrm{F}$ & OUPR 20290 \\
\hline Microlicia arenariaefolia DC. & Subshrub & $\mathrm{CR}$ & RB 41804 \\
\hline Microlicia avicularis Mart. ex Naudin & Subshrub & $\mathrm{CR}$ & OUPR 9986 \\
\hline Microlicia confertiflora Naudin & Subshrub & $\mathrm{CR}$ & OUPR 12815 \\
\hline Microlicia cordata (Spreng.) Cham. & Subshrub & $\mathrm{CR}$ & OUPR 6788 \\
\hline Microlicia crenulata (DC.) Mart. & Subshrub & $\mathrm{CR}$ & OUPR 20462 \\
\hline Microlicia euphorbioides Mart. & Subshrub & $\mathrm{CR}$ & OUPR 9996 \\
\hline Microlicia fasciculata Mart. ex Naudin & Subshrub & $\mathrm{CR}$ & HUFU 57696 \\
\hline Microlicia glazioviana Cogn. $\mathrm{EN}^{* *}$ & Subshrub & $\mathrm{CR}$ & OUPR 8802 \\
\hline Microlicia graveolens DC. & Subshrub & $\mathrm{CR}$ & HUFU 57687 \\
\hline Microlicia isophylla DC. & Subshrub & $\mathrm{CR}$ & OUPR 6056 \\
\hline Microlicia macrophylla Naudin & Subshrub & $\mathrm{CR}$ & OUPR 9968 \\
\hline Microlicia multicaulis Mart. ex Naudin & Subshrub & $\mathrm{CR}$ & RB 515443 \\
\hline Microlicia parvifolia Naudin & Subshrub & $\mathrm{CR}$ & NY 926143 \\
\hline Microlicia serpyllifolia D.Don & Subshrub & $\mathrm{CR}$ & OUPR 6396 \\
\hline Microlicia sp1 & Subshrub & $\mathrm{CR}$ & OUPR 6783 \\
\hline Microlicia sp2 & Subshrub & $\mathrm{CR}$ & OUPR 7785 \\
\hline Microlicia sp3 & Subshrub & $\mathrm{CR}$ & OUPR 9985 \\
\hline Ossaea cinnamomifolia (Naudin) Triana & Shrub & $\mathrm{F}$ & OUPR 2143 \\
\hline Ossaea congestiflora (Naudin) Cogn. & Shrub & $\mathrm{CR}$ & OUPR 6652 \\
\hline Ossaea coriacea (Naudin) Triana & Shrub & $\mathrm{F}$ & OUPR 5917 \\
\hline Pleroma aemula P.J.F.Guim., A.L.F.Oliveira \& R.Romer & Tree & $\mathrm{F}$ & RB 524426 \\
\hline Rhynchanthera grandiflora (Aubl.) DC. & Shrub & $\mathrm{CR}$ & OUPR 6652 \\
\hline Siphanthera arenaria (DC.) Cogn. & Subshrub & $\mathrm{CR}$ & HUFU 57654 \\
\hline Siphanthera paludosa (DC.) Cogn. & Subshrub & $\mathrm{CR}$ & OUPR 23552 \\
\hline Tibouchina canescens (D.Don) Cogn. & Tree & $\mathrm{F}$ & OUPR 1236 \\
\hline Tibouchina cardinalis (Humb. \& Bonpl.) Cogn. & Shrub & $\mathrm{CR}$ & OUPR 6160 \\
\hline Tibouchina cerastifolia Cogn. & Shrub & $\mathrm{CR}$ & UEC 187651 \\
\hline Tibouchina cisplatensis Cogn. & Subshrub & $\mathrm{CR}$ & OUPR 6162 \\
\hline Tibouchina collina (Naud.) Cogn. & Subshrub & $\mathrm{CR}$ & UPBC 70958 \\
\hline
\end{tabular}


MESSIAS, M.C.T.B. et al.

Table 1. Continued..

\begin{tabular}{|c|c|c|c|}
\hline Family / Species & Habit & Habitat & Voucher \\
\hline Tibouchina dendroides (Naudin) Cogn. & Shrub & CR & OUPR 6175 \\
\hline Tibouchina estrellensis (Raddi) Cogn. & Tree & $\mathrm{F}$ & OUPR 6178 \\
\hline Tibouchina fissinervia (Schrank \& Mart. ex DC.) Cogn. & Shrub & $\mathrm{F}$ & OUPR 1324 \\
\hline Tibouchina formosa Cogn. & Shrub & $\mathrm{CR}$ & OUPR 23555 \\
\hline Tibouchina fothergillae (Schrank \& Mart. ex DC.) Cogn. & Tree & $\mathrm{F}$ & OUPR 19076 \\
\hline Tibouchina frigidula (DC.) Cogn. & Subshrub & CR & OUPR 6198 \\
\hline Tibouchina gardneriana (Triana) Cogn. & Tree & $\mathrm{F}$ & HUFU 57919 \\
\hline Tibouchina gracilis (Bonpl.) Cogn. & Subshrub & $\mathrm{CR}$ & OUPR 6794 \\
\hline Tibouchina herbacea (DC.) Cogn. & Subshrub & $\mathrm{CR}$ & RB 524414 \\
\hline Tibouchina heteromalla (D.Don) Cogn. & Subshrub & $\mathrm{CR}$ & OUPR 6138 \\
\hline Tibouchina hieracioides (DC.) Cogn. & Subshrub & $\mathrm{CR}$ & HUFU 53795 \\
\hline Tibouchina martialis (Cham.) Cogn. & Shrub & $\mathrm{F}$ & OUPR 1267 \\
\hline Tibouchina martiusiana (DC.) Cogn. & Shrub & $\mathrm{CR}, \mathrm{F}$ & OUPR 1321 \\
\hline Tibouchina moricandiana Baill. & Shrub & $\mathrm{F}$ & MBM 233920 \\
\hline Tibouchina mutabilis (Vell.) Cogn. & Tree & $\mathrm{F}$ & RB 40788 \\
\hline Tibouchina semidecandra (Schrank \& Mart. ex DC.) Cogn. & Shrub & $\mathrm{CR}, \mathrm{F}$ & OUPR 6777 \\
\hline Tibouchina stenocarpa (Schrank \& Mart. ex DC.) Cogn. & Shrub & $\mathrm{CR}, \mathrm{F}$ & NY 941804 \\
\hline Tibouchina thereminiana (DC.) Cogn. & Shrub & $\mathrm{CR}$ & RB 98503 \\
\hline Tibouchina trichopoda (DC.) Baill. & Shrub & $\mathrm{CR}$ & OUPR 6488 \\
\hline Tibouchina valtherii Cogn. & Tree & $\mathrm{F}$ & RB 524426 \\
\hline Tibouchina sp & Shrub & $\mathrm{CR}$ & OUPR 4805 \\
\hline Trembleya calycina Cham. EX*, $\mathrm{EN}^{* *}$ & Shrub & $\mathrm{CR}$ & OUPR 6274 \\
\hline Trembleya laniflora (D.Don) Cogn. & Shrub & $\mathrm{CR}$ & OUPR 1365 \\
\hline Trembleya parviflora (D.Don) Cogn. & Shrub & $\mathrm{CR}, \mathrm{F}$ & OUPR 1378 \\
\hline Trembleya pentagona Naudin & Shrub & CR & OUPR 6425 \\
\hline Trembleya phlogiformis DC. & Shrub & $\mathrm{CR}$ & OUPR 1325 \\
\hline Trembleya tridentata Naudin & Shrub & $\mathrm{CR}$ & OUPR 1506 \\
\hline \multicolumn{4}{|l|}{ Meliaceae } \\
\hline Cabralea canjerana subsp. canjerana (Vell.) Mart. & Tree & $\mathrm{CR}, \mathrm{F}$ & OUPR 5531 \\
\hline Cabralea canjerana subsp. polytricha (A. Juss.) T.D.Penn. & Shrub & CR & OUPR 19180 \\
\hline \multicolumn{4}{|l|}{ Menispermaceae } \\
\hline Abuta sp & Liana & $\mathrm{F}$ & OUPR 7397 \\
\hline Cissampelos andromorpha DC. & Liana & $\mathrm{F}$ & OUPR 8991 \\
\hline \multicolumn{4}{|l|}{ Monimiaceae } \\
\hline Macropeplus ligustrinus (Tul.) Perkins & Shrub & $\mathrm{F}$ & OUPR 5470 \\
\hline Macropeplus schwackeanus (Perkins) I.Santos \& Peixoto VU* & Tree & $\mathrm{F}$ & BHCB 122307 \\
\hline Mollinedia glabra (Spreng.) Perkins & Tree & $\mathrm{F}$ & OUPR 21698 \\
\hline \multicolumn{4}{|l|}{ Moraceae } \\
\hline Dorstenia urceolata Schott & Subshrub & $\mathrm{F}$ & OUPR 1291 \\
\hline Ficus sp & Tree & $\mathrm{F}$ & OUPR 19179 \\
\hline Sorocea bonplandii (Baill.) W.C.Burger, Lanj. \& de Boer & Tree & $\mathrm{F}$ & OUPR 21335 \\
\hline \multicolumn{4}{|l|}{ Myrtaceae } \\
\hline Blepharocalyx salicifolius (Kunth) O.Berg & Shrub & CR & RB 276290 \\
\hline Calyptranthes pulchella DC. & Shrub & $\mathrm{CR}, \mathrm{F}$ & BHCB (FF Carmo 3484) \\
\hline Campomanesia adamantium (Cambess.) O.Berg & Shrub & CR & OUPR 1980 \\
\hline Campomanesia guaviroba (DC.) Kiaersk. & Shrub & $\mathrm{F}$ & $\begin{array}{c}\text { BHCB } \\
\text { (M.O. Bünger 543) }\end{array}$ \\
\hline Campomanesia pubescens (DC.) O.Berg. & Shrub & $\mathrm{CR}$ & OUPR 19392 \\
\hline Campomanesia rufa (O.Berg) Nied. & Shrub & $\mathrm{CR}, \mathrm{F}$ & OUPR 21145 \\
\hline Campomanesia simulans M.L.Kawas. & Tree & $\mathrm{F}$ & OUPR 20248 \\
\hline Eucalyptus grandis W.Hill & Tree & $\mathrm{F}$ & OUPR 20898 \\
\hline Eugenia brasiliensis Lam. & Shrub & $\mathrm{F}$ & SP 313342 \\
\hline Eugenia cerasiflora Miq. & Tree & $\mathrm{F}$ & BHCB 144673 \\
\hline Eugenia florida DC. & Shrub & $\mathrm{F}$ & BHCB 144297 \\
\hline Eugenia longipedunculata Nied. & Shrub & $\mathrm{CR}, \mathrm{F}$ & OUPR 21660 \\
\hline Eugenia ligustrina (Sw.) Willd. & Tree & $\mathrm{CR}, \mathrm{F}$ & OUPR 24025 \\
\hline Eugenia mosenii (Kausel) Sobral & Tree & $\mathrm{F}$ & OUPR 1435 \\
\hline Marlierea angustifolia (O.Berg) Mattos & Tree & $\mathrm{F}$ & SP 313316 \\
\hline
\end{tabular}


Table 1. Continued...

\begin{tabular}{|c|c|c|c|}
\hline Family / Species & Habit & Habitat & Voucher \\
\hline Marlierea antonia (O.Berg) D.Legrand & Tree & $\mathrm{F}$ & OUPR 1492 \\
\hline Marlierea excoriate Mart. & Tree & $\mathrm{F}$ & OUPR 21702 \\
\hline Marlierea laevigata (DC.) Kiaersk. & Tree & $\mathrm{F}$ & BHCB 144296 \\
\hline Marlierea tomentosa Cambess. & Tree & $\mathrm{F}$ & UEC 157769 \\
\hline Marlierea obscura O.Berg & Tree & $\mathrm{CR}, \mathrm{F}$ & OUPR 19162 \\
\hline Marlierea sp & Shrub & CR & OUPR 15617 \\
\hline Myrceugenia alpigena (DC.) Landrum & Shrub & $\mathrm{CR}, \mathrm{F}$ & OUPR 5721 \\
\hline Myrceugenia miersiana (Gardner) D.Legrand \& Kausel & Tree & $\mathrm{F}$ & OUPR 19166 \\
\hline Myrceugenia ovata (Hook. \& Arn.) O.Berg & Tree & $\mathrm{F}$ & ВHCB 107934 \\
\hline Myrcia amazonica DC. & Tree & $\mathrm{CR}, \mathrm{F}$ & OUPR 6207 \\
\hline Myrcia crocea Kiaersk. & Tree & F & OUPR 21705 \\
\hline Myrcia eriopus DC. & Shrub & $\mathrm{CR}, \mathrm{F}$ & OUPR 24032 \\
\hline Myrcia eriocalyx DC. & Shrub & $\mathrm{CR}, \mathrm{F}$ & OUPR 22798 \\
\hline Myrcia guianensis (Aubl.) DC. & Shrub & $\mathrm{CR}$ & OUPR 3733 \\
\hline Myrcia hartwegiana (O.Berg) Kiaersk. & Shrub & $\mathrm{CR}, \mathrm{F}$ & OUPR 22797 \\
\hline Myrcia hebepetala DC. & Shrub & F & OUPR 3819 \\
\hline Myrcia laruotteana Cambess. & Shrub & $\mathrm{F}$ & OUPR 23794 \\
\hline Myrcia lutescens Cambess. & Tree & $\mathrm{F}$ & BHCB 144300 \\
\hline Myrcia montana Cambess. & Shrub & $\mathrm{CR}$ & OUPR 21668 \\
\hline Myrcia mutabilis (O.Berg) N.Silveira & Tree & $\mathrm{CR}$ & OUPR 24034 \\
\hline Myrcia obovata (O.Berg) Nied. & Shrub & $\mathrm{CR}, \mathrm{F}$ & OUPR 4186 \\
\hline Myrcia pubiflora DC. & Subshrub & $\mathrm{CR}, \mathrm{F}$ & OUPR 21666 \\
\hline Myrcia pulchra (O.Berg) Kiaersk. & Shrub & $\mathrm{CR}$ & OUPR 21671 \\
\hline Myrcia retorta Cambess. & Shrub & $\mathrm{CR}, \mathrm{F}$ & OUPR 1432 \\
\hline Myrcia rufipes DC. & Shrub & $\mathrm{F}$ & OUPR 19169 \\
\hline Myrcia splendens (Sw.) DC. & Tree & $\mathrm{CR}, \mathrm{F}$ & OUPR 3569 \\
\hline Myrcia subalpestris DC. & Tree & $\mathrm{F}$ & MBM 227913 \\
\hline Myrcia subcordata DC. & Shrub & $\mathrm{CR}$ & OUPR 11332 \\
\hline Myrcia subverticillaris (O. Berg) Kiaersk. & Shrub & $\mathrm{CR}, \mathrm{F}$ & OUPR 22801 \\
\hline Myrcia tomentosa (Aubl.) DC. & Shrub & $\mathrm{F}$ & OUPR 6208 \\
\hline Myrcia vauthieriana O.Berg & Tree & $\mathrm{F}$ & OUPR 19172 \\
\hline Myrcia venulosa DC. & Shrub & $\mathrm{CR}, \mathrm{F}$ & OUPR 22296 \\
\hline Myrciaria floribunda (H.West ex Willd.) O.Berg & Shrub & $\mathrm{CR}, \mathrm{F}$ & OUPR 24035 \\
\hline Myrciaria glanduliflora (Kiaersk.) Mattos \& D.Legrand & Shrub & $\mathrm{CR}$ & VIC 20862 \\
\hline Plinia cauliflora (Mart.) Kausel & Tree & $\mathrm{F}$ & $\begin{array}{c}\text { BHCB } \\
\text { (M.O. Bünger 544) }\end{array}$ \\
\hline Psidium firmum O.Berg & Shrub & $\mathrm{CR}$ & OUPR 22799 \\
\hline Psidium myrtoides O.Berg & Tree & $\mathrm{F}$ & BHCB 161191 \\
\hline Psidium robustum O.Berg & Shrub & $\mathrm{CR}$ & OUPR 5810 \\
\hline Psidium rufum Mart. ex DC. & Tree & $\mathrm{F}$ & OUPR 20248 \\
\hline Siphoneugena crassifolia (DC.) Proença \& Sobral & Shrub & $\mathrm{F}$ & OUPR 19164 \\
\hline Siphoneugena densiflora O.Berg & Shrub & $\mathrm{CR}, \mathrm{F}$ & OUPR 19163 \\
\hline Siphoneugena dussii (Krug \& Urb.) Proença & Shrub & $\mathrm{CR}$ & OUPR 22329 \\
\hline Siphoneugena kiaerskoviana (Burret) Kausel & Tree & $\mathrm{CR}$ & RB 276300 \\
\hline \multicolumn{4}{|l|}{ Nyctaginaceae } \\
\hline Guapira opposita (Vell.) Reitz & Shrub & $\mathrm{F}$ & OUPR 17709 \\
\hline Guapira tomentosa (Casar.) Lundell & Shrub & $\mathrm{CR}$ & OUPR 1412 \\
\hline Neea $\mathrm{sp}$ & Shrub & $\mathrm{CR}$ & OUPR 17734 \\
\hline \multicolumn{4}{|l|}{ Ochnaceae } \\
\hline Luxemburgia nobilis Eichler ex Engl. & Shrub & $\mathrm{CR}$ & OUPR 6730 \\
\hline Luxemburgia octandra A.St.-Hill. & Shrub & $\mathrm{CR}$ & OUPR 6816 \\
\hline Ouratea floribunda (A.St.-Hil.) Engl. & Tree & $\mathrm{F}$ & OUPR 2265 \\
\hline Ouratea multiflora (Pohl) Engl. & Shrub & $\mathrm{CR}$ & RB 60921 \\
\hline Ouratea semiserrata (Mart. \& Nees) Engl. & Shrub & $\mathrm{CR}$ & OUPR 1372 \\
\hline Sauvagesia erecta $\mathrm{L}$. & Subshrub & $\mathrm{CR}$ & OUPR 5695 \\
\hline Sauvagesia vellozii (Vell. ex A.St.-Hil.) Sastre & Subshrub & $\mathrm{CR}, \mathrm{F}$ & UB 185430 \\
\hline \multicolumn{4}{|l|}{ Oleaceae } \\
\hline Jasminum mesnyi Hance & Shrub & AA & OUPR 19375 \\
\hline
\end{tabular}


MESSIAS, M.C.T.B. et al.

Table 1. Continued...

\begin{tabular}{|c|c|c|c|}
\hline Family / Species & Habit & Habitat & Voucher \\
\hline \multicolumn{4}{|l|}{ Onagraceae } \\
\hline Fuchsia coccinea Dryand. & Shrub & $\mathrm{CR}$ & RB 50293 \\
\hline Fuchsia regia (Vell.) Munz & Shrub & $\mathrm{CR}, \mathrm{F}$ & OUPR 1265 \\
\hline Ludwigia anastomosans (DC.) H.Hara & Shrub & $\mathrm{CR}$ & OUPR 1243 \\
\hline Ludwigia octovalvis (Jacq.) P.H.Raven & Subshrub & $\mathrm{CR}, \mathrm{F}$ & NY 1066833 \\
\hline \multicolumn{4}{|l|}{ Orchidaceae } \\
\hline Acianthera prolifera (Herb. ex Lindl.) Pridgeon \& M.W.Chase & Herb & $\mathrm{CR}$ & OUPR 1444 \\
\hline Acianthera teres (Lindl.) Borba & Herb & $\mathrm{CR}$ & OUPR 22796 \\
\hline Anacheilium allemanoides (Hoene) Pabst, Moutinho \& A.V.Pinto & Herb & $\mathrm{F}$ & OUPR 9621 \\
\hline Bifrenaria aureofulva Lindl. & Herb & $\mathrm{F}$ & BHCB 125888 \\
\hline Bifrenaria tyrianthina (Lodd.) Rchb.f. & Herb & CR & OUPR 9623 \\
\hline Cattleya bradei (Pabst) Van den Berg & Herb & $\mathrm{CR}$ & OUPR 3940 \\
\hline Cattleya cinnabarina (Bateman ex Lindl.) Van den Berg & Herb & $\mathrm{CR}$ & OUPR 7311 \\
\hline Cattleya crispata (Thunb.) Van den Berg & Herb & $\mathrm{CR}$ & OUPR 8730 \\
\hline Cattleya longipes (Rchb.f.) Van den Berg & Herb & $\mathrm{CR}$ & OUPR 8730 \\
\hline Christensonella subulata (Lindl.) Szlach. et al. & Herb & $\mathrm{CR}$ & OUPRPR 20226 \\
\hline Cleistes libonii (Rchb.f.) Schltr. & Herb & $\mathrm{CR}$ & OUPR 3943 \\
\hline Cleistes metallina (Barb. Rodr.) Schltr. & Herb & $\mathrm{CR}$ & OUPR 15540 \\
\hline Cleistes montana Gardner & Herb & $\mathrm{CR}$ & OUPR 1442 \\
\hline Cyrtopodium $\mathrm{sp}$ & Herb & $\mathrm{CR}$ & OUPR 9056 \\
\hline Dichaea cogniauxiana Schltr. & Herb & $\mathrm{CR}, \mathrm{F}$ & BHCB 125897 \\
\hline Epidendrum chlorinum Barb.Rodr. & Herb & CR & ВHCB 125899 \\
\hline Epidendrum dendrobioides Thunb. & Herb & $\mathrm{CR}$ & OUPR 3944 \\
\hline Epidendrum denticulatum Barb. Rodr. & Herb & $\mathrm{CR}$ & OUPR 3954 \\
\hline Epidendrum martianum Lindl. & Herb & $\mathrm{F}$ & OUPR 20465 \\
\hline Epidendrum rupicolum Cogn. & Herb & CR & OUPR 3951 \\
\hline Epidendrum secundum Jacq. & Herb & $\mathrm{CR}, \mathrm{F}$ & OUPR 8732 \\
\hline Epidendrum xanthinum Lindl. & Herb & $\mathrm{CR}, \mathrm{F}$ & OUPR 3937 \\
\hline Epistephium praestans Hoehne & Herb & $\mathrm{CR}$ & OUPR 1445 \\
\hline Erythrodes sp & Herb & CR & OUPR 22039 \\
\hline Galeandra junceoides Barb.Rodr. & Herb & $\mathrm{CR}$ & BHCB 40137 \\
\hline Gomesa planifolia (Lindl.) K1. \& Rchb.f. & Herb & $\mathrm{CR}$ & OUPR 3950 \\
\hline Gomesa ramosa (Lindl.) M.W.Chase \& N.H.Williams & Herb & CR & OUPR 3953 \\
\hline Gomesa recurva $\mathrm{R} . \mathrm{Br}$. & Herb & CR & OUPR 3950 \\
\hline Gomesa warmingii (Rchb.f.) M.W.Chase \& N.H.Williams VU* & Herb & $\mathrm{CR}$ & OUPR 3952 \\
\hline Grobya amherstiae Lindl. & Herb & $\mathrm{CR}$ & OUPR 3942 \\
\hline Habenaria caldensis Kraenzl. & Herb & $\mathrm{CR}$ & OUPR 3938 \\
\hline Habenaria hydrophila Barb.Rodr. & Herb & $\mathrm{CR}$ & RB 98594 \\
\hline Habenaria imbricata Lindl. & Herb & $\mathrm{CR}$ & BHCB 120315 \\
\hline Habenaria itaculumia Garay $\mathrm{CR}^{* *}$ & Herb & CR & OUPR 20469 \\
\hline Habenaria parviflora Lindl. & Herb & $\mathrm{CR}$ & OUPR 3947 \\
\hline Habenaria petalodes Lindl. & Herb & $\mathrm{CR}$ & OUPR 8253 \\
\hline Habenaria rodeiensis Barb.Rodr. & Herb & $\mathrm{CR}$ & OUPR 1443 \\
\hline Habenaria rupestris Poepp.\& Endl. & Herb & $\mathrm{CR}$ & OUPR 22046 \\
\hline Habenaria rupicola Barb.Rodr. & Herb & $\mathrm{CR}$ & OUPR 9715 \\
\hline Habenaria secundiflora Barb.Rodr. & Herb & $\mathrm{CR}$ & OUPR 14317 \\
\hline Habenaria subviridis Hoehne \& Schltr. & Herb & $\mathrm{CR}$ & OUPR 3948 \\
\hline Habenaria trifida Kunth & Herb & CR & UB 19884 \\
\hline Habenaria sp & Herb & $\mathrm{CR}$ & OUPR 18245 \\
\hline Hadrolaelia brevipedunculata (Cogn.) Chiron \& V.P.Castro VU** & Herb & CR & OUPR 13864 \\
\hline Hadrolaelia coccinea (Lindl.) Chiron \& V.P.Castro VU* & Herb & $\mathrm{CR}, \mathrm{F}$ & OUPR 13866 \\
\hline Koellensteinia tricolor (Lindl.) Rchb.f. & Herb & $\mathrm{F}$ & OUPR 19293 \\
\hline Malaxis excavata (Lindl.) Kuntze & Herb & $\mathrm{F}$ & OUPR 14273 \\
\hline Pelexia oestrifera (Rchb.f. \& Warm.) Schltr. & Herb & $\mathrm{CR}$ & BHCB 125691 \\
\hline Pelexia sp & Herb & $\mathrm{CR}$ & OUPR 1821 \\
\hline Prescottia glazioviana Cogn. & Herb & CR & OUPR 3945 \\
\hline Prescottia montana Barb.Rodr. & Herb & $\mathrm{CR}$ & OUPR 3946 \\
\hline Prescottia plantaginifolia Lindl. ex Hook. & Herb & $\mathrm{CR}$ & RB 193666 \\
\hline
\end{tabular}


Flora of Itacolomi State Park

Table 1. Continued...

\begin{tabular}{|c|c|c|c|}
\hline Family / Species & Habit & Habitat & Voucher \\
\hline Prosthechea allemanoides (Hoehne) W.E.Higgins & Herb & CR & BHCB 125925 \\
\hline Prosthechea moojenii (Pabst) W.E.Higgins & Herb & $\mathrm{CR}$ & RB 182939 \\
\hline Prosthechea vespa (Vell.) W.E.Higgins & Herb & $\mathrm{CR}$ & R 222290 \\
\hline Prostechea sp & Herb & $\mathrm{CR}$ & RB 193659 \\
\hline Scuticaria hadwenii (Lindl.) Planch. & Herb & $\mathrm{CR}$ & OUPR 13969 \\
\hline Skeptrostachys congestiflora (Cogn.) Garay & Herb & $\mathrm{CR}$ & OUPR 1447 \\
\hline Xylobium foveatum (Lindl.) Nicholson & Herb & $\mathrm{CR}$ & OUPR 3941 \\
\hline Zygopetalum brachypetalum Lindl. & Herb & $\mathrm{CR}$ & RB 98634 \\
\hline Zygopetalum maculatum (Kunth) Garay & Herb & $\mathrm{CR}, \mathrm{F}$ & RB 206763 \\
\hline Zygopetalum triste Barb.Rodr. & Herb & CR & RB 193667 \\
\hline Zygopetalum sp & Herb & $\mathrm{CR}$ & RB 193664 \\
\hline \multicolumn{4}{|l|}{ Orobanchaceae } \\
\hline Buchnera lavandulacea Cham. \& Schltdl. & Subshrub & $\mathrm{CR}$ & OUPR 12816 \\
\hline Castilleja arvensis Schltdl. \& Cham. & Subshrub & $\mathrm{F}$ & OUPR 7747 \\
\hline Escobedia grandiflora (L.f.) Kuntze & Subshrub & $\mathrm{CR}$ & OUPR 1320 \\
\hline Esterhazya macrodonta (Cham.) Benth. & Subshrub & $\mathrm{CR}$ & OUPR 1705 \\
\hline Esterhazya splendida J.C.Mikan & Subshrub & $\mathrm{CR}$ & OUPR 13474 \\
\hline Velloziella spathacea (Oliv.) Melch. & Subshrub & $\mathrm{CR}$ & OUPR 1269 \\
\hline \multicolumn{4}{|l|}{ Passifloraceae } \\
\hline Passiflora haematostigma Mart. ex Mast. & Liana & $\mathrm{CR}$ & OUPR 1416 \\
\hline Passiflora porophylla Vell. & Liana & $\mathrm{CR}$ & IAC 53474 \\
\hline Passiflora speciosa Gardner & Liana & $\mathrm{F}$ & OUPR 5156 \\
\hline Passiflora villosa Vell. & Liana & $\mathrm{CR}$ & OUPR 23350 \\
\hline Passiflora sp & Liana & $\mathrm{CR}$ & OUPR 20522 \\
\hline \multicolumn{4}{|l|}{ Phyllanthaceae } \\
\hline Phyllanthus fastigiatus Mart. ex Müll.Arg. & Subshrub & $\mathrm{CR}$ & OUPR 20172 \\
\hline Phyllanthus klotzschianus Müll.Arg. & Subshrub & $\mathrm{CR}$ & OUPR 1304 \\
\hline Phyllanthus niruri L. & Subshrub & $\mathrm{CR}$ & OUPR 6395 \\
\hline Phyllanthus obtusatus (Bilb.) Müll.Arg. & Subshrub & $\mathrm{CR}$ & NY 1064830 \\
\hline Phyllanthus roselus (Müll. Arg.) Müll.Arg. & Subshrub & $\mathrm{CR}$ & RB 277727 \\
\hline \multicolumn{4}{|l|}{ Phytolaccaceae } \\
\hline Phytolacca thyrsiflora Fenzl ex J.A.Schmidt & Subshrub & $\mathrm{CR}, \mathrm{F}$ & OUPR 7467 \\
\hline \multicolumn{4}{|l|}{ Pinaceae } \\
\hline Pinus sp & Tree & $\mathrm{F}$ & OUPR 22826 \\
\hline \multicolumn{4}{|l|}{ Piperaceae } \\
\hline Ottonia sp & Shrub & $\mathrm{F}$ & OUPR 20256 \\
\hline Peperomia alata Ruiz \& Pav. & Subshrub & $\mathrm{CR}$ & RB 399012 \\
\hline Peperomia blanda (Jacq.) H.B.K. & Subshrub & $\mathrm{F}$ & OUPR 6907 \\
\hline Peperomia cernuum Vell. & Shrub & $\mathrm{F}$ & NY 1021340 \\
\hline Peperomia corcovadensis Gardner & Subshrub & $\mathrm{CR}$ & BHCB 8246 \\
\hline Peperomia palcipila C.DC. & Subshrub & $\mathrm{CR}$ & OUPR 6889 \\
\hline Peperomia subrubrispica C.DC. & Subshrub & $\mathrm{CR}$ & RB 331623 \\
\hline Peperomia tenella (Sw.) A.Dietr. & Subshrub & $\mathrm{CR}$ & RB 54549 \\
\hline Peperomia tetraphylla (G.Forst.) Hook. \& Arn. & Subshrub & $\mathrm{CR}$ & OUPR 7368 \\
\hline Piper aduncum L. & Shrub & $\mathrm{CR}$ & OUPR 20294 \\
\hline Piper baptisianum C.DC. & Shrub & $\mathrm{F}$ & OUPR 1496 \\
\hline Piper cernuum Vell. & Shrub & $\mathrm{F}$ & UB 113500 \\
\hline Piper coccoloboides (Kunth) Kunth ex C.DC. & Shrub & $\mathrm{F}$ & OUPR 18261 \\
\hline Piper corcovadensis (Miq.) C.DC. & Shrub & $\mathrm{F}$ & OUPR 1312 \\
\hline Piper crassinervium Kunth & Shrub & $\mathrm{F}$ & OUPR 18262 \\
\hline Piper damazii C.DC. & Shrub & $\mathrm{F}$ & OUPR 1311 \\
\hline Piper lhotzkyanum Kunth & Shrub & $\mathrm{CR}$ & RB 357134 \\
\hline Piper regnellii (Miq.) C.DC. & Shrub & $\mathrm{F}$ & OUPR 18260 \\
\hline Piper richardiifolium (Kunth) Kunth ex C.DC. & Subshrub & $\mathrm{F}$ & MO 3079243 \\
\hline Piper solmsianum C.DC. & Shrub & $\mathrm{CR}$ & RB 54631 \\
\hline Piper tectoniaefolium (Kunth) Kunth ex C.DC. & Shrub & $\mathrm{F}$ & RB 331625 \\
\hline Piper umbellatum L. & Shrub & $\mathrm{F}$ & OUPR 6909 \\
\hline Piper sp1 & Shrub & $\mathrm{F}$ & OUPR 1310 \\
\hline
\end{tabular}


MESSIAS, M.C.T.B. et al.

Table 1. Continued...

\begin{tabular}{|c|c|c|c|}
\hline Family / Species & Habit & Habitat & Voucher \\
\hline Piper sp2 & Shrub & $\mathrm{F}$ & OUPR 6774 \\
\hline Piper sp3 & Shrub & $\mathrm{F}$ & OUPR 20253 \\
\hline \multicolumn{4}{|l|}{ Plantaginaceae } \\
\hline Plantago guilleminiana Decne. & Herb & $\mathrm{CR}$ & NY 423565 \\
\hline Plantago tomentosa Lam. & Herb & $\mathrm{CR}$ & OUPR 12586 \\
\hline Scoparia dulcis L. & Subshrub & $\mathrm{CR}$ & OUPR 25230 \\
\hline Stemodia lobata J.A.Schmidt VU* & Subshrub & $\mathrm{CR}$ & OUPR 17291 \\
\hline Stemodia microphylla J.A.Schmidt & Herb & $\mathrm{CR}$ & OUPR 17733 \\
\hline Stemodia trifoliata (Link) Rchb. & Subshrub & $\mathrm{CR}$ & VIC 54548 \\
\hline \multicolumn{4}{|l|}{ Poaceae } \\
\hline Andropogon bicornis L. & Herb & $\mathrm{CR}$ & OUPR 15614 \\
\hline Andropogon ingratus Hack. & Herb & $\mathrm{CR}$ & CEN 28917 \\
\hline Andropogon leucostachyus Kunth. & Herb & $\mathrm{CR}$ & OUPR 22791 \\
\hline Andropogon ternatus (Spreng.) Nees & Herb & $\mathrm{CR}$ & MBM 45496 \\
\hline Andropogon virgatus Desv. ex Ham. & Herb & $\mathrm{CR}$ & OUPR 20463 \\
\hline Andropogon virginicum (L.) Spreng. & Herb & $\mathrm{CR}$ & OUPR 1498 \\
\hline Apochloa chnoodes (Trin.) Zuloaga \& Morrone & Herb & $\mathrm{CR}$ & BHCB 72595 \\
\hline Apochloa lorea (Trin.) Zuloaga \& Morrone & Herb & $\mathrm{CR}$ & OUPR 17748 \\
\hline Apochloa molinioides (Trin.) Zuloaga \& Morrone & Herb & $\mathrm{CR}$ & NY 381729 \\
\hline Apochloa poliophylla (Renvoize \& Zuloaga) Zuloaga \& Morrone & Herb & $\mathrm{CR}$ & OUPR 7291 \\
\hline Aristida recurvata Kunth & Herb & $\mathrm{CR}$ & NY 861291 \\
\hline Aristida torta (Nees) Kunth & Herb & CR & OUPR 17149 \\
\hline Aulonemia sp & Herb & $\mathrm{CR}$ & UEC 171751 \\
\hline Axonopus aureus P.Beauv. & Herb & $\mathrm{CR}$ & MO 861610 \\
\hline Axonopus fissifolius (Raddi) Kuhlm. & Herb & CR & NY 861744 \\
\hline Axonopus pellitus (Nees ex Trin.) Hitchc. \& Chase & Herb & $\mathrm{CR}$ & OUPR 15600 \\
\hline Axonopus polystachyus G.A.Black & Herb & $\mathrm{CR}$ & MO 1577833 \\
\hline Axonopus pressus (Nees ex Steud.) Parodi & Herb & $\mathrm{CR}$ & OUPR 15599 \\
\hline Axonopus siccus (Nees) Kuhlm. & Herb & $\mathrm{CR}$ & OUPR 20177 \\
\hline Axonopus sp & Herb & CR & OUPR 15599 \\
\hline Cenchrus polystachios (L.) Morrone & Herb & $\mathrm{CR}$ & OUPR 1501 \\
\hline Chascolytrum calotheca (Trin.) Essi,Longhi-Wagner \& Souza-Chies & Herb & $\mathrm{CR}$ & OUPR 15956 \\
\hline Chusquea attenuata (Döll) L.G.Clark EN** & Herb & CR & MBM 27517 \\
\hline Chusquea nutans L.G.Clark & Herb & CR & OUPR 16241 \\
\hline Chusquea pinifolia (Nees) Nees & Herb & $\mathrm{CR}$ & BHCB 8211 \\
\hline Colanthelia sp & Herb & $\mathrm{CR}$ & BHCB 107925 \\
\hline Danthonia sp & Herb & $\mathrm{CR}$ & OUPR 16613 \\
\hline Dichanthelium sabulorum (Lam.) Gould \& C.A.Clark & Herb & $\mathrm{CR}$ & MO 1919711 \\
\hline Dichanthelium sciurotoides (Zuloaga \& Morrone) Davidse & Herb & $\mathrm{F}$ & OUPR 6763 \\
\hline Dichanthelium stigmosum (Trin.) Zuloaga & Herb & $\mathrm{F}$ & BHCB 133922 \\
\hline Dichanthelium superatum (Hack.) Zuloaga & Herb & $\mathrm{F}$ & NY 864620 \\
\hline Digitaria hololeuca Henrard & Herb & $\mathrm{CR}$ & UB 14109 \\
\hline Digitaria insularis (L.) Mez ex Ekman & Herb & CR & OUPR 16608 \\
\hline Digitaria pampinosa Henrard $\mathrm{EN}^{* *}$ & Herb & $\mathrm{CR}$ & NY 862167 \\
\hline Digitaria sejuncta (Hack. ex Pilg.) Henrard & Herb & $\mathrm{CR}$ & NY 862170 \\
\hline Echinochloa crusgalli (L.) P.Beauv. & Herb & $\mathrm{CR}$ & OUPR 15952 \\
\hline Echinolaena inflexa (Poir.) Chase & Herb & $\mathrm{CR}$ & OUPR 16612 \\
\hline Elionurus muticus (Spreng.) Kuntze & Herb & $\mathrm{CR}$ & MBM 27827 \\
\hline Eragrostis articulata (Schrank) Nees & Herb & $\mathrm{CR}$ & NY 862503 \\
\hline Eragrostis pilosa (L.) P.Beauv. & Herb & $\mathrm{CR}$ & OUPR 1497 \\
\hline Eragrostis rufescens Schrad. ex Schult. & Herb & $\mathrm{CR}$ & OUPR 12768 \\
\hline Eragrostis solida Nees & Herb & $\mathrm{CR}$ & OUPR 22785 \\
\hline Eriochrysis holcoides (Nees) Kuhlm. & Herb & $\mathrm{CR}$ & OUPR 13512 \\
\hline Eriochrysis sp & Herb & $\mathrm{CR}$ & BHCB 721 \\
\hline Ichnanthus bambusiflorus (Trin.) Döll & Herb & $\mathrm{CR}$ & OUPR 10775 \\
\hline Ichnanthus calvescens (Nees ex Trin.) Döll & Herb & CR & OUPR 20228 \\
\hline Ichnanthus pallens (Sw.) Munro ex Benth. & Herb & $\mathrm{CR}$ & OUPR 6764 \\
\hline Isachne $\mathrm{sp}$ & Herb & $\mathrm{CR}$ & OUPR 17559 \\
\hline
\end{tabular}


Table 1. Continued...

\begin{tabular}{|c|c|c|c|}
\hline Family / Species & Habit & Habitat & Voucher \\
\hline Melinis minutiflora P.Beauv. & Herb & $\mathrm{CR}$ & OUPR 15615 \\
\hline Merostachys fischeriana Rupr. ex Döll & Herb & $\mathrm{CR}, \mathrm{F}$ & HUEFS 147721 \\
\hline Merostachys sp & Herb & $\mathrm{F}$ & OUPR 24844 \\
\hline Mesosetum ferrugineum (Trin.) Chase & Herb & CR & OUPR 14124 \\
\hline Otachyrium versicolor (Döll) Henrard & Herb & $\mathrm{CR}$ & OUPR 22782 \\
\hline Panicum campestre Nees ex Trin. & Herb & CR & NY 863761 \\
\hline Panicum sellowii Nees & Herb & $\mathrm{F}$ & OUPR 6762 \\
\hline Parodiolyra micrantha (Kunth) Davidse \& Zuloaga & Herb & $\mathrm{F}$ & OUPR 1499 \\
\hline Paspalum conjugatum P.J.Bergius & Herb & CR & NY 862363 \\
\hline Paspalum coryphaeum Trin. & Herb & CR & OUPR 23303 \\
\hline Paspalum eucomum Nees ex Trin. & Herb & CR & OUPR 15612 \\
\hline Paspalum glaucescens Hack. & Herb & CR & MO 2969469 \\
\hline Paspalum hyalinum Nees ex Trin. & Herb & CR & OUPR 22784 \\
\hline Paspalum juergensii Hack. & Herb & CR & SP 199550 \\
\hline Paspalum mandiocanum Trin. & Herb & CR & MO 2972192 \\
\hline Paspalum multicaule Poir & Herb & $\mathrm{CR}$ & OUPR 22784 \\
\hline Paspalum pilosum Lam. & Herb & CR & OUPR 22790 \\
\hline Paspalum polyphyllum Nees ex Trin. & Herb & CR & OUPR 12819 \\
\hline Paspalum pumilum Nees & Herb & CR & NY 895924 \\
\hline Paspalum thrasyoides (Trin.) S.Denham & Herb & CR & MBM 27480 \\
\hline Paspalum $\mathrm{sp}$ & Herb & CR & OUPR 22793 \\
\hline Pseudechinolaena polystachya (Kunth) Stapf & Herb & $\mathrm{F}$ & OUPR 6762 \\
\hline Rugoloa pilosa (Sw.) Zuloaga & Herb & $\mathrm{CR}, \mathrm{F}$ & OUPR 7270 \\
\hline Schizachyrium sanguineum (Retz.) Alston & Herb & $\mathrm{CR}$ & OUPR 22786 \\
\hline Schizachyrium tenerum Nees & Herb & CR & OUPR 22787 \\
\hline Setaria parviflora (Poir.) M.Kerguelen & Herb & CR & MO 2985867 \\
\hline Setaria tenacissima Schrad. & Herb & CR & NY 906251 \\
\hline Sporobolus ciliatus J.Presl & Herb & CR & RB 239283 \\
\hline Sporobolus piliferus (Trin.) Kunth & Herb & $\mathrm{CR}$ & NY 896366 \\
\hline Steinchisma laxum (Sw.) Zuloaga & Herb & CR & NY 863853 \\
\hline Trichanthecium cyanescens (Nees ex Trin.) Zuloaga \& Morrone & Herb & CR & OUPR 15621 \\
\hline Trichanthecium pseudisachne (Mez) Zuloaga \& Morrone & Herb & CR & OUPR 22789 \\
\hline Trichanthecium wettsteinii (Hack.) Zuloaga \& Morrone & Herb & CR & OUPR 22783 \\
\hline \multicolumn{4}{|l|}{ Polygalaceae } \\
\hline Asemeia monninoides (Kunth) J.F.B.Pastore \& J.R.Abbott & Subshrub & $\mathrm{CR}$ & RB 398973 \\
\hline Asemeia violacea (Aubl.) F.B.Pastore \& J.R.Abbott & Subshrub & CR & OUPR 13589 \\
\hline Caamembeca laureola (A.St.-Hil. \& Moq.) J.F.B.Pastore & Subshrub & $\mathrm{CR}$ & NY 634708 \\
\hline Caamembeca oxyphylla (DC.) J.F.B.Pastore & Subshrub & $\mathrm{CR}$ & OUPR 1246 \\
\hline Polygala cuspidata DC. & Subshrub & $\mathrm{CR}$ & BHCB 8271 \\
\hline Polygala glochidiata Kunth & Subshrub & CR & BHCB 122262 \\
\hline Polygala paniculata $\mathrm{L}$. & Subshrub & $\mathrm{CR}$ & OUPR 13595 \\
\hline Polygala timoutou Aubl. & Subshrub & $\mathrm{CR}$ & OUPR 13601 \\
\hline \multicolumn{4}{|l|}{ Polygonaceae } \\
\hline Fallopia convolvulus (L.) A.Löve & Subshrub & CR & OUPR 7765 \\
\hline Homalocladium platycladum (F. Muell.) L.H.Bailey & Subshrub & $\mathrm{CR}$ & OUPR 9076 \\
\hline Persicaria capitata (Buch.-Ham. ex D.Don) H.Gros & Subshrub & AA & OUPR 19311 \\
\hline Persicaria hydropiperoides (Michx.) Small & Subshrub & $\mathrm{CR}$ & NY 863998 \\
\hline Persicaria meisneriana (Cham. \& Schltdl.) M.Gómez & Subshrub & $\mathrm{CR}$ & BHCB 8288 \\
\hline \multicolumn{4}{|l|}{ Portulacaceae } \\
\hline Portulaca mucronata Link & Subshrub & AA & NY 3413603 \\
\hline \multicolumn{4}{|l|}{ Primulaceae } \\
\hline Ardisia solanacea (Poir.) Roxb. & Subshrub & $\mathrm{CR}$ & OUPR 20271 \\
\hline Cybianthus itacolomyensis M.Lisboa \& Badini & Shrub & $\mathrm{F}$ & OUPR 5856 \\
\hline Lysimachia arvensis (L.) U.Manns \& Anderb. & Subshrub & AA & OUPR 6376 \\
\hline Myrsine congesta (Sw.) Pipoly EN ${ }^{* * *}$ & Shrub & $\mathrm{F}$ & RB 39981 \\
\hline Myrsine coriacea (Sw.) R.Br. ex Roem. \& Schult. & Shrub & $\mathrm{F}$ & OUPR 1315 \\
\hline Myrsine emarginella Miq. & Shrub & CR & RB 380828 \\
\hline Myrsine gardneriana A.DC. & Tree & $\mathrm{F}$ & OUPR 19154 \\
\hline
\end{tabular}


MESSIAS, M.C.T.B. et al.

Table 1. Continued...

\begin{tabular}{|c|c|c|c|}
\hline Family / Species & Habit & Habitat & Voucher \\
\hline Myrsine lancifolia Mart. & Shrub & CR & OUPR 12581 \\
\hline Myrsine lineata (Mez) Imkhan. & Tree & $\mathrm{F}$ & RB 277645 \\
\hline Myrsine squarrosa (Mez) M.F.Freitas \& Kin.-Gouv. & Shrub & $\mathrm{CR}$ & OUPR 1484 \\
\hline Myrsine umbellata Mart. & Shrub & $\mathrm{CR}, \mathrm{F}$ & OUPR 1399 \\
\hline Myrsine villosissima Mart. $\mathrm{EN}^{* *}$ & Shrub & $\mathrm{F}$ & OUPR 5555 \\
\hline \multicolumn{4}{|l|}{ Proteaceae } \\
\hline Euplassa rufa (Loes.) Sleumer & Shrub & $\mathrm{F}$ & OUPR 25098 \\
\hline Panopsis multiflora (Schott ex Spreng.) Ducke EN* & Tree & $\mathrm{F}$ & OUPR 1249 \\
\hline Roupala montana Aubl. var. montana & Shrub & $\mathrm{CR}$ & OUPR 1314 \\
\hline Roupala montana var. brasiliensis (Klotzsch) K.S.Edwards & Tree & $\mathrm{F}$ & OUPR 13429 \\
\hline \multicolumn{4}{|l|}{ Ranunculaceae } \\
\hline Clematis dioica $\mathrm{L}$. & Liana & $\mathrm{F}$ & OUPR 1355 \\
\hline \multicolumn{4}{|l|}{ Rhamnaceae } \\
\hline Crumenaria sp & Subshrub & $\mathrm{CR}$ & BHCB 41485 \\
\hline Reissekia smilacina (Sm.) Steud. & Liana & $\mathrm{F}$ & OUPR 20272 \\
\hline \multicolumn{4}{|l|}{ Rosaceae } \\
\hline Eriobotrya japonica (Thunb.) Lindl. & Tree & AA & OUPR 19309 \\
\hline Pirus communis L. & Tree & AA & OUPR 7479 \\
\hline Prunus sellowii Koehne & Tree & $\mathrm{F}$ & NY 922649 \\
\hline Rubus brasiliensis Mart. & Shrub & $\mathrm{F}$ & OUPR 1373 \\
\hline Rubus erythrocladus Mart. ex Hook.f. & Shrub & $\mathrm{F}$ & OUPR 1873 \\
\hline Rubus rosifolius $\mathrm{Sm}$. & Subshrub & AA & OUPR 12585 \\
\hline \multicolumn{4}{|l|}{ Rubiaceae } \\
\hline Amaioua guianensis Aubl. & Tree & $\mathrm{F}$ & BHCB 41498 \\
\hline Augusta longifolia (Spreng.) Rehder & Shrub & CR & OUPR 17752 \\
\hline Bathysa australis (A.St.-Hil.) K.Schum. & Shrub & $\mathrm{F}$ & OUPR 19289 \\
\hline Bathysa cuspidata (A.St.-Hil.) Hook.f. ex K.Schum. & Shrub & $\mathrm{F}$ & OUPR 1356 \\
\hline Borreria capitata (Ruiz \& Pav.) DC. & Subshrub & $\mathrm{CR}$ & OUPR 12796 \\
\hline Borreria verticillata (L.) G.Mey. & Subshrub & $\mathrm{AA}, \mathrm{CR}$ & OUPR 1250 \\
\hline Coccocypselum aureum (Spreng.) Cham. \& Schltdl. & Subshrub & $\mathrm{CR}, \mathrm{F}$ & OUPR 6792 \\
\hline Coccocypselum erythrocephalum Cham. \& Schltdl. & Subshrub & $\mathrm{CR}, \mathrm{F}$ & OUPR 6792 \\
\hline Coccocypselum lanceolatum (Ruiz \& Pav.) Pers. & Subshrub & $\mathrm{F}$ & RB 526340 \\
\hline Coccocypselum pedunculare Cham. \& Schltdl. & Subshrub & $\mathrm{CR}$ & RB 324887 \\
\hline Coccocypselum $\mathrm{sp}$ & Subshrub & $\mathrm{F}$ & OUPR 23254 \\
\hline Cordiera concolor (Cham.) Kuntze & Tree & $\mathrm{F}$ & IAC 52606 \\
\hline Cordiera elliptica (Cham.) Kuntze & Tree & $\mathrm{F}$ & OUPR 19173 \\
\hline Coussarea nodosa (Benth.) Müll.Arg. & Shrub & $\mathrm{F}$ & NY 396634 \\
\hline Coussarea sp & Tree & $\mathrm{F}$ & OUPR 19174 \\
\hline Declieuxia fruticosa (Will. ex Roem. \& Schult.) Kuntze & Subshrub & $\mathrm{CR}$ & RB 190168 \\
\hline Diodia saponariifolia (Cham. \& Schltdl.) K.Schum. & Subshrub & CR & NY397199 \\
\hline Emmeorhiza umbellata (Spreng.) K.Schum. & Subshrub & $\mathrm{CR}$ & BHCB 41487 \\
\hline Galianthe brasiliensis (Spreng.) E.L.Cabral \& Bacigalupo & Subshrub & $\mathrm{CR}$ & NY 950040 \\
\hline Galianthe verbenoides (Cham. \& Schltdl.) Griseb. & Subshrub & CR & OUPR 19379 \\
\hline Galium hirtum Lam. & Subshrub & $\mathrm{CR}$ & OUPR 19385 \\
\hline Galium hypocarpium (L.) Endl. ex Griseb. subsp. hypocarpium & Subshrub & $\mathrm{CR}$ & OUPR 1483 \\
\hline Galium noxium (A.St.-Hil.) Dempster & Subshrub & $\mathrm{CR}$ & SP 140254 \\
\hline Galium sellowianum (Cham.) Walp. & Subshrub & $\mathrm{CR}$ & OUPR 19383 \\
\hline Galium sp & Subshrub & CR & RB 318466 \\
\hline Hillia parasitica Jacq. & Shrub & $\mathrm{CR}$ & RB 318466 \\
\hline Ixora brevifolia Benth. & Shrub & $\mathrm{CR}$ & RB 110957 \\
\hline Manettia luteorubra (Vell.) Benth. & Liana & $\mathrm{CR}$ & OUPR 1285 \\
\hline Palicourea marcgravii A.St.-Hil. & Shrub & $\mathrm{F}$ & OUPR 1402 \\
\hline Palicourea tetraphylla Cham. \& Schltdl. & Subshrub & $\mathrm{F}$ & OUPR 1262 \\
\hline Posoqueria acutifolia Mart. & Shrub & CR & OUPR 19384 \\
\hline Posoqueria latifolia (Rudge) Schult. & Shrub & $\mathrm{CR}, \mathrm{F}$ & OUPR 19382 \\
\hline Psychotria hispidula Standl. ex Steyerm. & Shrub & CR & OUPR 19380 \\
\hline Psychotria paludosa Müll.Arg. EN*** & Subshrub & $\mathrm{F}$ & OUPR 7395 \\
\hline Psychotria purpurascens Müll.Arg. & Shrub & $\mathrm{F}$ & BHCB 41496 \\
\hline
\end{tabular}


Table 1. Continued...

\begin{tabular}{|c|c|c|c|}
\hline Family / Species & Habit & Habitat & Voucher \\
\hline Psychotria sessilis Vell. & Shrub & CR & RB 324889 \\
\hline Psychotria stachyoides Benth. & Subshrub & $\mathrm{F}$ & UEFS 35882 \\
\hline Psychotria subtriflora Müll.Arg. & Shrub & $\mathrm{F}$ & RB 324891 \\
\hline Psychotria vellosiana Benth. & Tree & $\mathrm{F}$ & OUPR 19175 \\
\hline Psychotria $\mathrm{sp} 1$ & Shrub & $\mathrm{F}$ & OUPR 6765 \\
\hline Psychotria $\mathrm{sp} 2$ & Shrub & $\mathrm{F}$ & RB 40224 \\
\hline Psyllocarpus laricoides Mart. \& Zucc. & Subshrub & $\mathrm{CR}$ & OUPR 7285 \\
\hline Remijia ferruginea (A.St.-Hil.) DC. & Shrub & $\mathrm{CR}$ & OUPR 8333 \\
\hline Rudgea nodosa (Cham.) Benth. & Shrub & $\mathrm{F}$ & RB 324880 \\
\hline Rudgea sessilis (Vell.) Müll.Arg. EN ${ }^{* * *}$ & Shrub & $\mathrm{CR}$ & OUPR 1317 \\
\hline \multicolumn{4}{|l|}{ Rutaceae } \\
\hline Dictyoloma vandellianum A.Juss. & Tree & $\mathrm{F}$ & OUPR 1763 \\
\hline Zanthoxylum rhoifolium Lam. & Tree & $\mathrm{F}$ & OUPR 2274 \\
\hline \multicolumn{4}{|l|}{ Salicaceae } \\
\hline Abatia americana (Gardner) Eichler & Shrub & $\mathrm{CR}, \mathrm{F}$ & OUPR 13612 \\
\hline Casearia arborea (Rich.) Urb. & Shrub & $\mathrm{CR}$ & OUPR 9078 \\
\hline Casearia lasiophylla Eichler & Tree & $\mathrm{CR}$ & OUPR 15311 \\
\hline Casearia selloana Eichler & Shrub & $\mathrm{CR}, \mathrm{F}$ & OUPR 6424 \\
\hline Casearia sylvestris Sw. & Tree & $\mathrm{F}$ & OUPR 8049 \\
\hline Xylosma serrata (Sw.) Krug \& Urb. & Shrub & $\mathrm{CR}$ & OUPR 5155 \\
\hline \multicolumn{4}{|l|}{ Sapindaceae } \\
\hline Cupania emarginata Cambess. & Tree & $\mathrm{F}$ & OUPR 1368 \\
\hline Cupania vernalis Cambess. & Tree & $\mathrm{F}$ & OUPR 1487 \\
\hline Matayba marginata Radlk. & Shrub & $\mathrm{CR}$ & OUPR 8344 \\
\hline Paullinia carpopoda Cambess. & Liana & $\mathrm{CR}, \mathrm{F}$ & OUPR 1438 \\
\hline Serjania elegans Cambess. & Liana & $\mathrm{F}$ & OUPR 1254 \\
\hline Serjania fuscifolia Radlk. & Liana & $\mathrm{F}$ & OUPR 1388 \\
\hline Serjania $\mathrm{sp}$ & Liana & $\mathrm{F}$ & OUPR 4804 \\
\hline \multicolumn{4}{|l|}{ Sapotaceae } \\
\hline Sarcaulus brasiliensis (A.DC.) Eyma & Tree & $\mathrm{CR}, \mathrm{F}$ & OUPR 22774 \\
\hline \multicolumn{4}{|l|}{ Scrophulariaceae } \\
\hline Buddleja brasiliensis J.Jacq. & Subshrub & $\mathrm{CR}$ & OUPR 19312 \\
\hline \multicolumn{4}{|l|}{ Siparunaceae } \\
\hline Siparuna arianeae V.Pereira & Shrub & $\mathrm{F}$ & OUPR 18915 \\
\hline Siparuna brasiliensis (Spreng.) A.DC. & Shrub & $\mathrm{F}$ & OUPR 1316 \\
\hline Siparuna guianensis Aubl. & Shrub & $\mathrm{F}$ & OUPR 20285 \\
\hline \multicolumn{4}{|l|}{ Smilacaceae } \\
\hline Smilax elastica Griseb. & Liana & $\mathrm{F}$ & RB 409613 \\
\hline Smilax oblongifolia Pohl ex Griseb. & Liana & $\mathrm{CR}$ & OUPR 16579 \\
\hline Smilax quinquenervia Vell. & Liana & $\mathrm{CR}$ & OUPR 5776 \\
\hline Smilax sp1 & Liana & $\mathrm{CR}$ & OUPR 9444 \\
\hline Smilax sp2 & Liana & $\mathrm{F}$ & OUPR 9974 \\
\hline \multicolumn{4}{|l|}{ Solanaceae } \\
\hline Acnistus arborescens (L.) Schltdl. & Tree & $\mathrm{F}$ & OUPR 7376 \\
\hline Aureliana brasiliana (Hunz.) Barboza \& Hunz. & Tree & $\mathrm{F}$ & OUPR 25103 \\
\hline Aureliana fasciculata (Vell.) Sendtn. & Shrub & $\mathrm{F}$ & OUPR 20213 \\
\hline Aureliana picta (Mart.) I.M.C.Rodrigues \& Stehmann. & Shrub & $\mathrm{CR}, \mathrm{F}$ & OUPR 23971 \\
\hline Aureliana velutina Sendtn. & Shrub & $\mathrm{CR}, \mathrm{F}$ & OUPR 25141 \\
\hline Browallia americana $\mathrm{L}$. & Subshrub & AA & OUPR 24835 \\
\hline Brunfelsia brasiliensis (Spreng.) L.B.Sm. \& Downs & Shrub & $\mathrm{CR}, \mathrm{F}$ & OUPR 25118 \\
\hline Brunfelsia hydrangeiformis (Pohl) Benth. & Shrub & $\mathrm{F}$ & OUPR 25687 \\
\hline Brunfelsia uniflora (Pohl) D.Don & Shrub & $\mathrm{F}$ & OUPR 10426 \\
\hline Capsicum schottianum Sendtn. & Shrub & $\mathrm{CR}, \mathrm{F}$ & OUPR 24870 \\
\hline Capsicum $\mathrm{sp}$ & Shrub & $\mathrm{CR}, \mathrm{F}$ & OUPR 25113 \\
\hline Cestrum bracteatum Link \& Otto & Shrub & $\mathrm{CR}, \mathrm{F}$ & OUPR 25138 \\
\hline Cestrum schlechtendahlii G.Don & Shrub & F & OUPR 25300 \\
\hline Dyssochroma viridiflora (Sims) Miers & Shrub & $\mathrm{CR}, \mathrm{F}$ & OUPR 25114 \\
\hline Nicotiana alata Link \& Otto & Subshrub & AA & MBM 233808 \\
\hline
\end{tabular}


Table 1. Continued...

\begin{tabular}{|c|c|c|c|}
\hline Family / Species & Habit & Habitat & Voucher \\
\hline Nicotiana bonariensis Lehm. & Subshrub & $\mathrm{CR}$ & OUPR 25602 \\
\hline Nicotiana langsdorfii Weinm. & Subshrub & AA & OUPR 24834 \\
\hline Physalis pubescens $\mathrm{L}$. & Subshrub & AA & OUPR 24898 \\
\hline Schwenckia americana Rooyen ex L. & Subshrub & $\mathrm{CR}, \mathrm{F}$ & OUPR 24945 \\
\hline Solanum americanum Mill. & Subshrub & $\mathrm{CR}, \mathrm{F}$ & OUPR 25140 \\
\hline Solanum argenteum Dunal & Shrub & $\mathrm{CR}, \mathrm{F}$ & OUPR 25088 \\
\hline Solanum campaniforme Roem. \& Schult. & Shrub & $\mathrm{F}$ & OUPR 25219 \\
\hline Solanum cernuиm Vell. & Shrub & $\mathrm{F}$ & OUPR 25194 \\
\hline Solanum cinnamomeum Sendtn. & Tree & $\mathrm{F}$ & OUPR 24854 \\
\hline Solanum cladotrichum Dunal & Tree & $\mathrm{CR}, \mathrm{F}$ & OUPR 20085 \\
\hline Solanum granulosoleprosum Dunal & Tree & $\mathrm{CR}, \mathrm{F}$ & OUPR 25086 \\
\hline Solanum inodorum Vell. & Liana & $\mathrm{F}$ & OUPR 25212 \\
\hline Solanum intermedium Sendtn. & Shrub & $\mathrm{CR}, \mathrm{F}$ & OUPR 25083 \\
\hline Solanum leptostachys Dunal & Shrub & $\mathrm{CR}, \mathrm{F}$ & OUPR 24796 \\
\hline Solanum luridifuscescens Bitter & Shrub & $\mathrm{F}$ & OUPR 25589 \\
\hline Solanum lycocarpum A.St.-Hil. & Shrub & $\mathrm{CR}, \mathrm{F}$ & OUPR 25192 \\
\hline Solanum palinacanthum Dunal & Shrub & $\mathrm{AA}$ & OUPR 22863 \\
\hline Solanum paniculatum $\mathrm{L}$. & Shrub & $\mathrm{AA}, \mathrm{CR}$ & OUPR 24897 \\
\hline Solanum pseudocapsicum L. & Subshrub & F & OUPR 25590 \\
\hline Solanum pseudoquina A.St.-Hil. & Tree & F & OUPR 24860 \\
\hline Solanum refractifolium Schltdl. & Subshrub & $\mathrm{CR}$ & OUPR 25076 \\
\hline Solanum reptans Bunbury & Subshrub & $\mathrm{CR}$ & OUPR 24869 \\
\hline Solanum sisymbriifolium Lam. & Subshrub & $\mathrm{F}$ & OUPR 4809 \\
\hline Solanum subumbellatum Vell. & Shrub & $\mathrm{CR}$ & OUPR 24941 \\
\hline Solanum swartzianum Roem. \& Schult. & Tree & $\mathrm{CR}, \mathrm{F}$ & OUPR 25139 \\
\hline Solanum vaillantii Dunal & Shrub & $\mathrm{CR}, \mathrm{F}$ & OUPR 24861 \\
\hline Solanum velleum Thunb. & Tree & $\mathrm{CR}, \mathrm{F}$ & OUPR 25122 \\
\hline Solanum viarum Dunal & Shrub & F & OUPR 25100 \\
\hline Solanum viscosissimum Sendtn. EN** & Liana & $\mathrm{F}$ & OUPR 4806 \\
\hline \multicolumn{4}{|l|}{ Styracaceae } \\
\hline Styrax aureus Mart. & Tree & $\mathrm{CR}$ & OUPR 9080 \\
\hline Styrax ferrugineus Nees \& Mart. & Tree & $\mathrm{CR}$ & UB 157565 \\
\hline Styrax latifolius Pohl & Tree & $\mathrm{CR}$ & OUPR 19464 \\
\hline Styrax maninul B.Walln. VU* & Shrub & CR,F & OUPR 24626 \\
\hline \multicolumn{4}{|l|}{ Symplocaceae } \\
\hline Symplocos arbutifolia Casar & Shrub & $\mathrm{CR}, \mathrm{F}$ & OUPR 24966 \\
\hline Symplocos celastrinea Mart. & Shrub & $\mathrm{F}$ & OUPR 19308 \\
\hline Symplocos falcata Brand & Tree & $\mathrm{F}$ & OUPR 21357 \\
\hline \multicolumn{4}{|l|}{ Theaceae } \\
\hline Camellia sinensis (L.) Kuntze. & Shrub & $\mathrm{CR}, \mathrm{F}$ & OUPR 1364 \\
\hline Laplacea fructicosa (Schrad.) Kobuski & Tree & $\mathrm{F}$ & OUPR 12210 \\
\hline \multicolumn{4}{|l|}{ Thymelaeaceae } \\
\hline Daphnopsis brasiliensis Mart. & Shrub & F & OUPR 13423 \\
\hline \multicolumn{4}{|l|}{ Trigoniaceae } \\
\hline Trigonia paniculata Warm. & Liana & $\mathrm{F}$ & OUPR 7269 \\
\hline \multicolumn{4}{|l|}{ Urticaceae } \\
\hline Boehmeria sp & Subshrub & $\mathrm{F}$ & OUPR 19292 \\
\hline Cecropia hololeuca Miq. & Tree & $\mathrm{F}$ & OUPR 29493 \\
\hline Cecropia pachystachya Trécul & Tree & $\mathrm{F}$ & OUPR 7426 \\
\hline Urera baccifera (L.) Gaudich. ex Wedd. & Shrub & $\mathrm{F}$ & OUPR 1362 \\
\hline \multicolumn{4}{|l|}{ Velloziaceae } \\
\hline Barbacenia damaziana Beauverd & Herb & $\mathrm{CR}$ & OUPR 3627 \\
\hline Barbacenia flava Mart. ex Schult. \& Schult.f. & Herb & $\mathrm{CR}$ & OUPR 19297 \\
\hline Barbacenia tomentosa Mart. & Herb & $\mathrm{CR}$ & OUPR 3264 \\
\hline Vellozia compacta Mart. ex Schult. \& Schult.f. & Subshrub & $\mathrm{CR}$ & OUPR 1382 \\
\hline Vellozia graminea Pohl & Herb & $\mathrm{CR}$ & OUPR 3626 \\
\hline Vellozia hirsuta Goethart \& Henrard & Herb & $\mathrm{CR}$ & RB 190101 \\
\hline Vellozia sp & Subshrub & $\mathrm{CR}$ & OUPR 1383 \\
\hline
\end{tabular}


Table 1. Continued...

\begin{tabular}{|c|c|c|c|}
\hline Family / Species & Habit & Habitat & Voucher \\
\hline \multicolumn{4}{|l|}{ Verbenaceae } \\
\hline Lantana camara L. & Shrub & $\mathrm{CR}$ & OUPR 6803 \\
\hline Lantana fucata Lindl. & Subshrub & $\mathrm{CR}$ & OUPR 19376 \\
\hline Lantana trifolia $\mathrm{L}$. & Subshrub & $\mathrm{CR}$ & OUPR 23691 \\
\hline Lantana $\mathrm{sp}$ & Shrub & $\mathrm{F}$ & OUPR 20293 \\
\hline Lippia hermannioides Cham. & Shrub & $\mathrm{CR}$ & OUPR 15534 \\
\hline Stachytarpheta cayennensis (Rich.) Vahl & Subshrub & $\mathrm{CR}$ & OUPR 12580 \\
\hline Stachytarpheta commutata Schauer & Subshrub & $\mathrm{CR}$ & OUPR 7398 \\
\hline Stachytarpheta glabra Cham. & Subshrub & $\mathrm{CR}$ & OUPR 20180 \\
\hline Stachytarpheta jamaicensis (L.) Vahl & Subshrub & $\mathrm{CR}$ & OUPR 20295 \\
\hline Stachytarpheta mexiae Moldenke & Shrub & $\mathrm{CR}$ & RB 98723 \\
\hline Verbena litoralis Kunth & Subshrub & $\mathrm{CR}$ & NY 956732 \\
\hline \multicolumn{4}{|l|}{ Violaceae } \\
\hline Anchietea exaltata Eichler & Liana & $\mathrm{F}$ & OUPR 19300 \\
\hline Anchietea pyrifolia (Mart.) G.Don & Liana & $\mathrm{F}$ & OUPR 8248 \\
\hline Viola subdimidiata A.St.-Hil. & Subshrub & $\mathrm{F}$ & OUPR 23469 \\
\hline \multicolumn{4}{|l|}{ Vitaceae } \\
\hline Cissus verticillata (L.) Nicolson \& C.E. Jarvis & Liana & $\mathrm{CR}$ & OUPR 9092 \\
\hline \multicolumn{4}{|l|}{ Vochysiaceae } \\
\hline Qualea lundii (Warm.) Warm. & Tree & $\mathrm{F}$ & OUPR 21356 \\
\hline Vochysia dasyantha Warm. & Tree & $\mathrm{F}$ & OUPR 4818 \\
\hline Vochysia emarginata (Vahl) Poir. & Shrub & $\mathrm{CR}$ & OUPR 7793 \\
\hline Vochysia rectiflora Warm. & Tree & $\mathrm{F}$ & OUPR 19343 \\
\hline Vochysia thyrsoidea Pohl & Tree & $\mathrm{F}$ & OUPR 1261 \\
\hline Vochysia tucanorum Mart. & Tree & $\mathrm{CR}, \mathrm{F}$ & OUPR 19176 \\
\hline \multicolumn{4}{|l|}{ Winteraceae } \\
\hline Drimys angustifolia Miers & Shrub & $\mathrm{F}$ & MBM 264545 \\
\hline Drimys brasiliensis Miers & Shrub & $\mathrm{F}$ & OUPR 6798 \\
\hline Drimys winteri J.R.Forst. \& G.Forst. & Tree & $\mathrm{F}$ & OUPR 9093 \\
\hline \multicolumn{4}{|l|}{ Xyridaceae } \\
\hline Xyris asperula Mart. & Herb & $\mathrm{CR}$ & OUPR 1485 \\
\hline Xyris augusto-coburgii Szyszyl. ex Beck & Herb & $\mathrm{CR}$ & OUPR 14470 \\
\hline Xyris graminosa Pohl ex Mart. & Herb & $\mathrm{CR}$ & OUPR 15954 \\
\hline Xyris hymenachne Mart. & Herb & $\mathrm{CR}$ & RB 277721 \\
\hline Xyris macrocephala Vahl & Herb & $\mathrm{CR}$ & OUPR 20230 \\
\hline Xyris peregrina Malme & Herb & $\mathrm{CR}$ & OUPR 20459 \\
\hline Xyris plantaginea Mart. & Herb & $\mathrm{CR}$ & OUPR 20468 \\
\hline Xyris schizachne Mart. & Herb & $\mathrm{CR}$ & RB 275396 \\
\hline Xyris teres Alb.Nilsson & Herb & $\mathrm{CR}$ & OUPR 17290 \\
\hline Xyris trachyphylla Mart. & Herb & $\mathrm{CR}$ & OUPR 4453 \\
\hline Xyris sp1 & Herb & $\mathrm{CR}$ & OUPR 16393 \\
\hline Xyris sp2 & Herb & $\mathrm{CR}$ & OUPR 20461 \\
\hline \multicolumn{4}{|l|}{ Zingiberaceae } \\
\hline Hedychium coronarium J. König & Herb & $\mathrm{CR}$ & OUPR 22339 \\
\hline
\end{tabular}

The families with higher species richness were Asteraceae (323), Fabaceae (154), Melastomataceae (131), Poaceae (84), Orchidaceae (62), Myrtaceae (58) and Bignoniaceae (57), represent more than $75 \%$ of the total sampled species (Figure 2). The richness of these families was also reported in other campos rupestres (Harley \& Simmons 1986, Giulietti et al. 1987, 1997, Pirani et al. 1994, Conceição \& Giulietti 2002, Conceição \& Pirani 2005, Jacobi \& Carmo 2012, Messias et al. 2012, Forzza et al. 2013). Fabaceae, Myrtaceae, Melastomataceae and Asteraceae are also species-rich families in Espinhaço Range forests (Kamino et al. 2008). However, comparing with the data presented by these authors, Bignoniaceae and Solanaceae presented higher tree species richness in ISP than in other
Espinhaço Range forests. Almeida et al. (2014) studying Asteraceae of ISP, described three new species and considered this Park as the richest Brazilian one for Asteraceae. Many families (29) are represented by only one species, what has been reported in other surveys along the Espinhaço Range (Giulietti et al. 1987, 1997, Pirani et al.1994, 2003).

The campos rupestres represent $51 \%$ of the total area, followed by the forest with $40 \%$ and anthropogenic disturbed areas with $9 \%$ (Figure 1). The vegetation seems to be closely associated with geology, geomorphology, depth and moisture of the soil, as well as the degree of anthropogenic impact, resulting in a variety of different physiognomies (Figure 3), similarly to other montane areas (Benites et al. 2007; Schaefer et al. 2016). The campos 


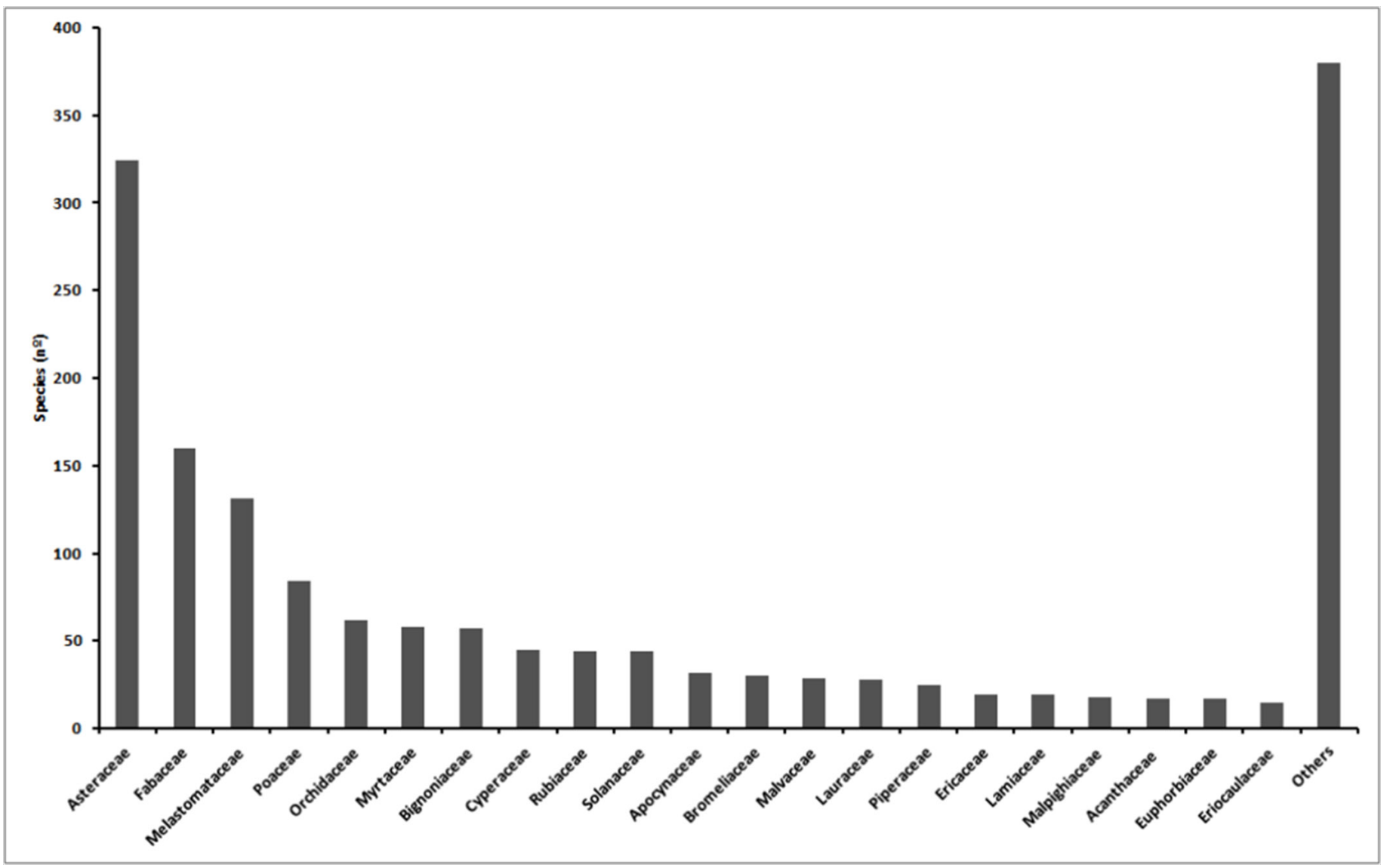

Figure 2. Fanerogamic species-richest families in the Itacolomi State Park, Ouro Preto and Mariana, Minas Gerais, Brazil.

rupestres occur in the highest parts of the ISP, always higher than $1000 \mathrm{~m}$, and the forests mostly in the lower ones, from $700 \mathrm{~m}$ to $1300 \mathrm{~m}$, usually along streams and slopes with deeper soil.

The forests at Itacolomi State Park have the same pattern of the forests along the Espinhaço Range (Harley 1995), varying considerably both in composition and structure in response to the geo-climatic conditions, often resulting in a large heterogeneity of patterns, even in a relatively small area. Thus, as described by Kamino et al. (2008), a riparian forest on the valleys may be evergreen, while the adjacent forest on the slope is semideciduous. In fact, Pedreira \& Sousa (2011) described a swampy evergreen forest patch in Itacolomi State Park, surrounded by semideciduous forests, with well-drained soils. In spite of the existence of this small scale variation in Itacolomi State Park forest fragments, they were only characterized in this work by the general feature as "montane forest".

Forest ecosystems with striking seasonality of rainfall or low soil water retention often drive high proportions of deciduous and semideciduous species in their communities (Reich \& Borchet 1984, Lenza \& Klink 2006). However, the fog events, common in the studied area, may favor the coexistence of species with different phenological strategies (Valim et al. 2013). Thus, care must be taken when generalizing the terminology "seasonal semideciduous forests" (Scolforo et al. 2008) for the forest of this park and similar surrounding areas.

The greatest species richness occurs in campos rupestres (almost 70\%), with $61 \%$ of species occurring exclusively in this phytophysiognomy. The campo rupestre is a very old mountaintop ecosystem, being a museum of ancient lineages as well as a cradle of continuing diversification of endemic lineages (Silveira et al. 2016). The astonishing species richness found in the campos rupestres is favoured by the high environmental diversity, wide latitudinal and altitudinal range, isolation and influence of different vegetation domains (Giulietti et al. 1997, Jacobi et al. 2007, Silveira et al. 2016).

The forests house about $36 \%$ of the species and $7 \%$ of the species occur in both physiognomies (forest and campos rupestres) like Eremanthus erythropappus, Schinus terebinthifolius, Clethra scabra, Vochysia tucanorum, among others. Some species occur just in anthropogenic disturbed areas $(1.8 \%)$ (Table 1, Figure 4). Some species found in the forests like Hedyosmum brasiliense, Laplacea fructicosa, Drimys brasiliensis, Weinmamania pauliniiflora and Ocotea percoriacea are typical of Brazilian montane forests (CRIA 2016).

The subshrubs represented $30 \%$ of the species, followed by shrubs (25\%), herbs (19\%), trees (13\%), lianas (12\%) and few palms and epiphytes (Figure 5). Shrubby species occur in both forest and campos rupestres, explaining the higher richness of this habit. In addition, the southern portion of the Espinhaço Range is more strongly influenced by the Atlantic Forest than the central/northern parts, leading to a higher proportion of phanerophytic species in campos rupestres (Echternacht et al. 2011). Most of the herbs occur in campos rupestres, mainly from Poaceae and Orchidaceae families. The epiphytes seem to be sub sampled since the montane forests of the region are reported as having a greater richness of this life-form (Ferreira, 2011).

Some parts of the forest are mono-dominated by Eremanthus erythropappus, known locally as candeal, similar to the other areas of Espinhaço Range (Oliveira-Filho \& Fluminhan-Filho 1999, Kamino et al. 2008). Most of these areas are in primary succession after fire occurrence.

The environmental impact in the ISP region dates back to the early seventeenth century, with gold mining. Later, with the decline of gold 

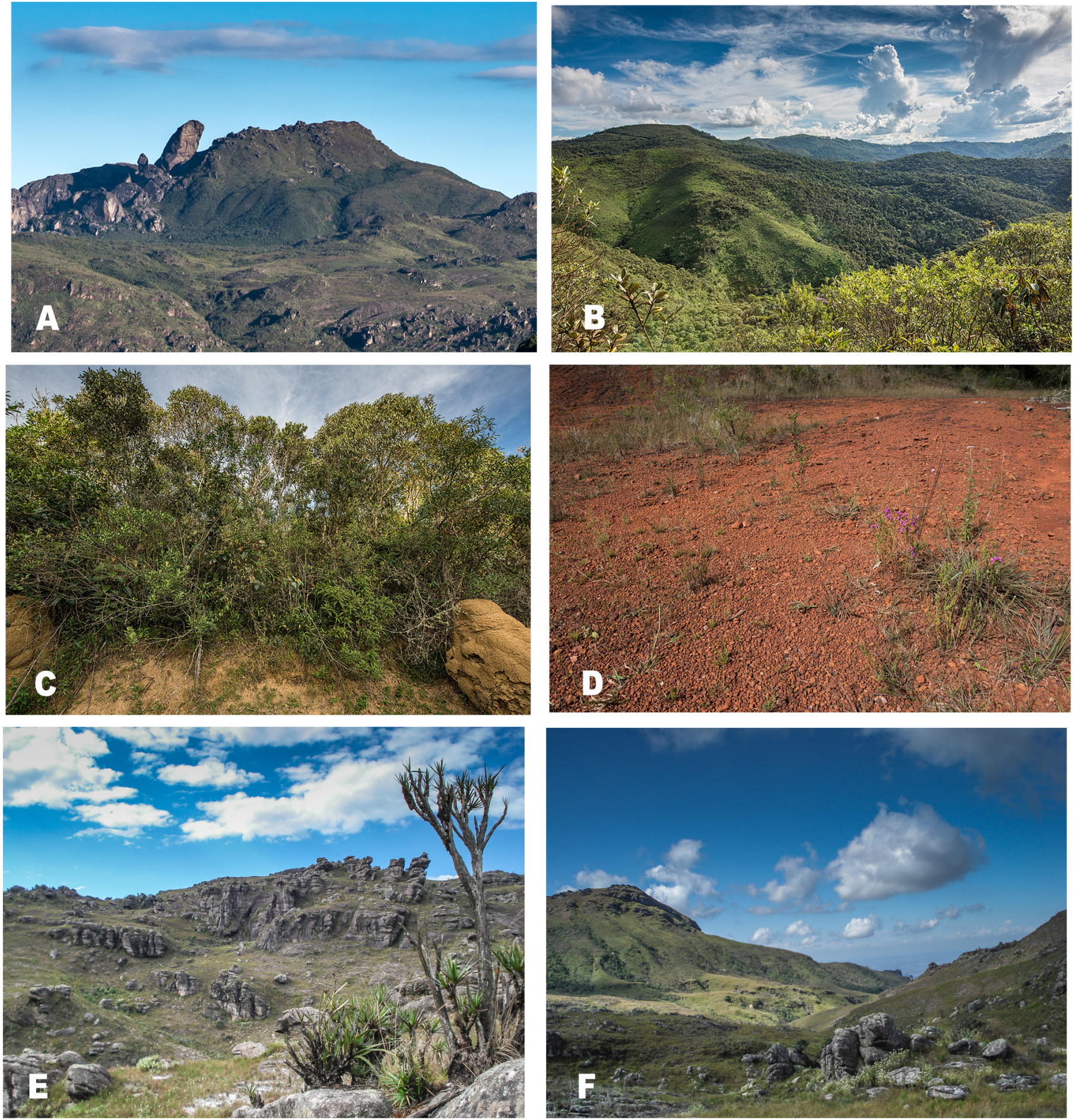

Figure 3. Phytophysiognomies of Itacolomi State Park, Ouro Preto and Mariana, Minas Gerais, Brazil. A. Itacolomi Peak surrounded by campos rupestres; B. Partial view of the Itacolomi State Park (ISP), with campos rupestres and forest patches; C. Monodominant forest made up of "Candeia" (Eremanthus erythropappus); D. Partial view of a ferruginous campo rupestre; E-F. Quartzitic campos rupestres.

exploration, some farms in this area focused on agriculture, especially tea (Cammelia sinensis), Eucalyptus plantation for charcoal production and cattle. The tea was introduced in this area in the eighteenth and nineteenth centuries, playing a key role in the local economy. This activity lasted until the 50s, when the plantations were then abandoned (Fujaco et al. 2010). Thus, individuals of this species are still found in the vicinity of the old farm "Fazenda São José do Manso", where there was the largest planted area. Other significant anthropogenic impact occurred in the second half of XX century, with the aluminum (bauxite) mining. In addition, more recently urban sprawl has become one of the main threats alongside the Park, compromising the integrity of the surrounding environment (Fujaco et al. 2010).

Most of the exotic phanerogamic species occur in these anthropogenic disturbed areas. The most aggressive ones are Eucalyptus grandis, 


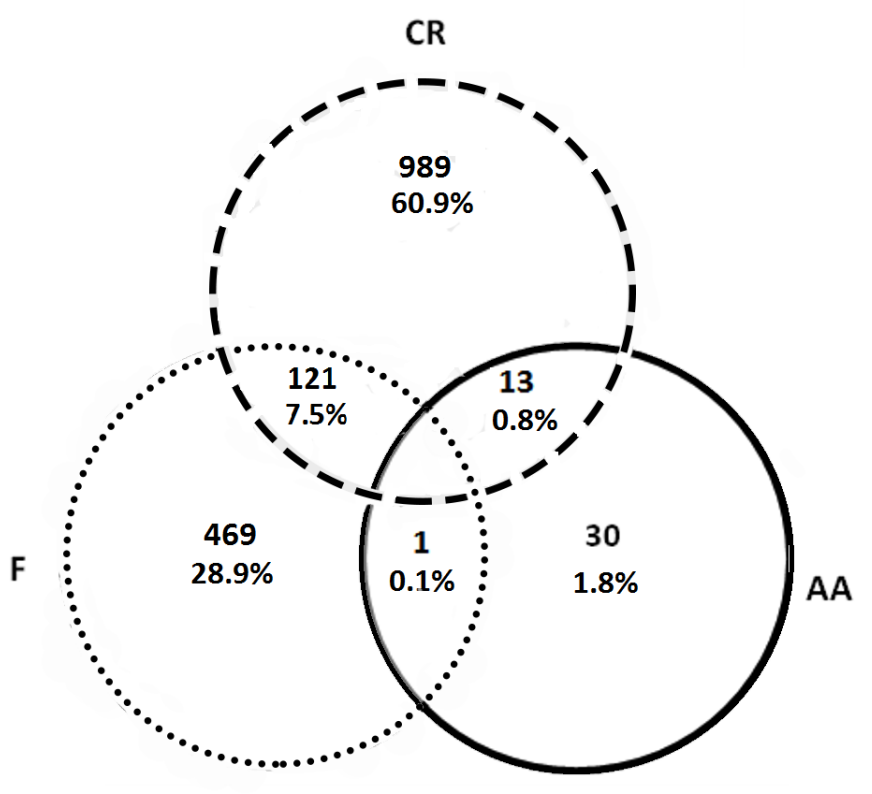

Figure 4. Venn diagram showing the number of shared and exclusive phanerogamic species in the different habitats at Itacolomi State Park, Ouro Preto and Mariana, Minas Gerais, Brazil. AA= Antropogenic disturbed areas, $\mathrm{CR}=$ Campos rupestres, $\mathrm{F}=$ Montane forests.

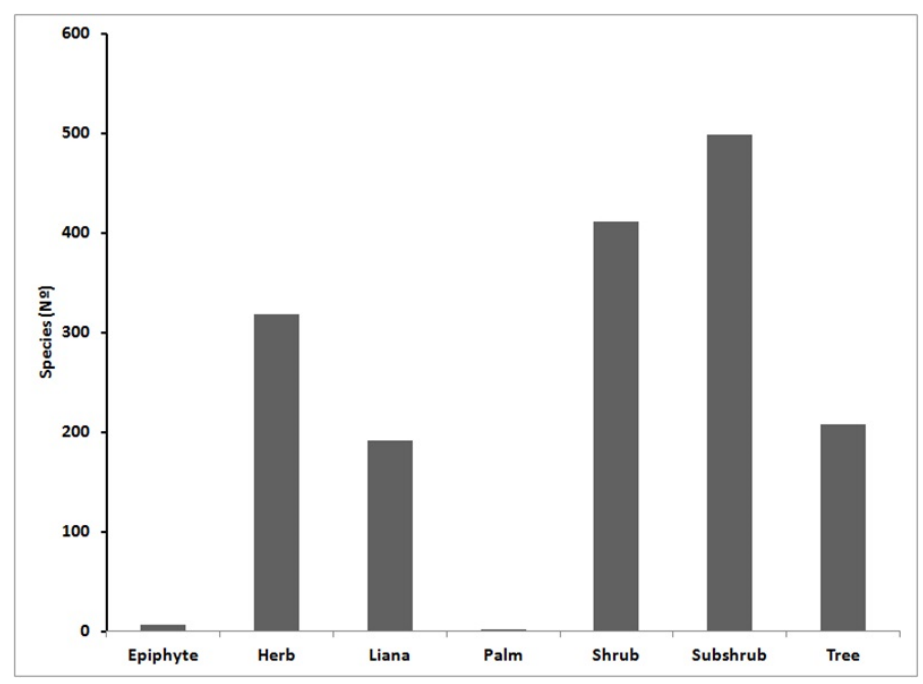

Figure 5. Growth habit of the recorded phanerogamic species of the Itacolomi State Park, Ouro Preto and Mariana, Minas Gerais, Brazil.

Hedychium coronarium and Melinis minutiflora. They have shown fast grown and high dispersal ability, easily increasing their population and spreading to other surrounding areas. Other exotic species observed didn't seem to present much competitiveness like Cammelia sinensis (tea), some ornamental plants like Hydrangea macrophylla, Impatiens balsamina and Rhododendron indicum. Probably, because most of the cultivated species are not able to grow in these metaliferous and nutrient-poor soils (Fernandes et al. 2016; Schaefer et al. 2016). Little is still known about biological invasion on mountaintop complexes where the threat of invasive species is very substantial (Wilson et al. 2016). Thus, more research is necessary about this subject.

Although Araucaria brasiliensis was considered initially as a naturally occurring species in this region (Mello-Barreto 1942, Hueck 1953), the observation of aerial photographs showed that the older individuals of this species were aligned, suggesting they were grown in this area. Further studies are still needed to clarify if this species is native to the Itacolomi State Park area.

A total of 79 species are threatened with extinction, being 57 species listed on the red list of Minas Gerais State (COPAM 1997), 40 figure on the Brazilian Red List (MMA 2014) and two others from IUCN Red List (2015). Most of them are described as vulnerable or endangered. However, some species are listed as critically endangered (CR) like Stevia hilarii, Valeriana organensis, Ocotea felix and Habenaria itaculumia on The Brazilian Red List (Table 1). Even though Trichogonia eupatorioides (=Trichogonia martii) and Trembleya calycina are described as probably extinct in the red list of the state of Minas Gerais (COPAM 1997), there are recent collections of these species in the herbarium databases (CRIA 2016). Other surveys along the Espinhaço Range have shown the same pattern, with many threatened species (Giulietti \& Pirani 1988, Pirani et al. 1994, Echternacht et al. 2011). The campos rupestres show a high concentration of endemic species, some of them occur in small population (Giulietti \& Pirani 1988, Echternacht et al. 2011). Thus, they are very vulnerable to extinction.

Some species are endemic to the Itacolomi State Park: Habenaria itaculumia and Heterocondylus itacolumiensis, in the campos rupestres and Cybianthus itacolomyensis in the forest. Other species are known as endemic to Espinhaço Range, in campos rupestres, like: Chamaecrista dentata, $C$. hedysaroides, $C$. rotundata, Cryptanthus schwackeanus, Mimosa aurivillus var. aurivillus, Aspilia caudata and Richterago hatschbachii (Flora do Brasil 2016, CRIA 2016).

The high flora diversity of this Park, harboring more than $12 \%$ of the Angiosperm species known for Minas Gerais State (BFG 2015), as well as the many endemic and endangered species, shows the value of this conservation unit as one of the most important in the state of Minas Gerais and Brazil. The knowledge of phanerogamic flora of ISP can help the Management Plan of this Unit of Conservation, contribute to future works related in this area and also improve the understanding of the Minas Gerais and Brazilian flora.

\section{Acknowledgements}

This paper is dedicated to the memory of Jorge Luiz da Silva. The authors wish to thank to FAPEMIG and IEF/PROFLORESTA by the financial support and also to the taxonomists A. Rapini (Apocynaceae), G.S.S. Almeida and J. Nakajima (Asteraceae), V.F. Dutra (Fabaceae), D. Medeiros (Euphorbiaceae), P.L. Moraes (Lauraceae), M. Sobral and M.O.Bünger (Myrtaceae), M.A.M. Azevedo (Passifloraceae), M.F. Freitas (Primulaceae), R. Marquete (Salicaceae), J.R. Stehmann (Solanaceae), J.L. Aranha-Filho (Symplocaceae). Acknowledgements are also given to Alberto V. M. Matos (IEF) for his assistance during field work.

\section{References}

ALMEIDA, G.S.S., CARVALHO-OKANO, R.M., NAKAJIMA, J.N. \& GARCIA, F.C.P. 2014. Asteraceae Dumort nos campos rupestres do Parque Estadual do Itacolomi, Minas Gerais, Brasil: Barnadesieae e Mutisieae. Rodriguésia 65:311-328.

ÁlVARES, C.A., STAPE, J.L., SENTElHAS, P.C., GONÇALVES, J.L.M. \& SPAROVEK, G. 2013. Köppen's climate classification map for Brazil. Meteorol Z. 22:711-728.

ALVES, R.J.V. 1990. The Orchidaceae of Itacolomi State Park in Minas Gerais, Brazil. Acta Bot. Bras. 4:65-72.

ALVES, R.J.V. \& KOLBEK, J. 2009. Summit vascular flora of Serra de São José, Minas Gerais, Brazil. Check List 5:35-73.

BATALHA, M.A. 2011. O cerrado não é um bioma. Biota Neotrop. 11:21-24. 
BATISTA, J.A.N., BIANCHETTI, L.B., NOGUEIRA, R.E., PELLIZZARO, K.F. \& FERREIRA, F.E. 2004. The genus Habenaria (Orchidaceae) in the Itacolomi State Park, Minas Gerais, Brazil. Sitientibus, Série Ciências Biológicas 4:25-36.

BENITES, V.M., SCHAEFER, C.E.G.R., SIMAS, F.N.B. \& SANTOS, H.G. 2007. Soils associated with rock outcrops in the Brazilian mountain ranges Mantiqueira and Espinhaço. Rev. Bras. Bot. 30:569-577.

BFG 2015. Growing knowledge: an overview of seed plant diversity in Brazil. Rodriguésia 66:1085-1113.

BÜNGER, M.O., SCALON, V.R., SOBRAL, M. \& STEHMANN, J.R. 2012. Myrtaceae no Parque Estadual do Itacolomi, Minas Gerais, Brasil. Rodriguésia 63:857-881.

CARMO, F.F. \& JACOBI, C.M. 2012 The cangas of the Iron Quadrangle. In Floristic diversity of the Quadrilátero Ferrífero cangas (C.M. Jacobi \& F.F. Carmo, Eds.) IDM, Belo Horizonte, p.14-30

CASTAÑEDA, C. 1993. Caracterização geológica e geomorfológica do Parque Estadual de Itacolomi. Projeto Itacolomi, Convênio IEF/UFOP/BIRD. Technical Report. Universidade Federal de Ouro Preto, Ouro Preto.

CHASE, M.W. \& REVEAL, J.L. 2009. A phylogenetic classification of the land plants to accompany APG III. Bot. J. Lin. Soc. 161:122-127.

CONCEIÇÃO, A.A. \& GIULIETTI, A.M. 2002. Composição florística e aspectos estruturais de campo rupestre em dois platôs do Morro do Pai Inácio, Chapada Diamantina, Bahia, Brasil. Hoehnea 29:37-48.

CONCEIÇÃO, A.A. \& PIRANI, J.R. 2005. Delimitação de habitats em campos rupestres na Chapada Diamantina, Bahia: Substratos, composição florística e aspectos estruturais. Bol. Bot. Univ. São Paulo 23:85-111.

COPAM. 1997. Deliberação COPAM no 85, 30 de outubro de 1997. Conselho Estadual de Política Ambiental, Belo Horizonte.

COSER, T.S., PAULA, C.C. \& WENDT, T. 2010. Bromeliaceae Juss. nos campos rupestres do Parque Estadual do Itacolomi, Minas Gerais, Brasil. Rodriguésia 61:261-280

COSTA, C.M.R., HERMANN, G., MARTINS, C.S., LINS, L.V. \& LAMAS, I.R. 1998. Biodiversidade em Minas Gerais: um atlas para sua conservação. Fundação Biodiversitas, Belo Horizonte.

COUTINHO, L.M. 2006. O conceito de bioma. Acta Bot. Bras. 20:1-11.

CRIA Centro de Referência em Informação Ambiental. 2016. speciesLink. http:// splink.cria.org.br/ (last access in July 1th, 2016).

DORR, J.V.N. 1969. Physiographic, stratigraphic and structural development of the Quadrilátero Ferrífero. Professional Paper, US Geological Survey 641-A, Washington.

DUTRA, V.F., MESSIAS, M.C.T.B. \& GARCIA, F.C.P. 2005. Papilionoideae (Leguminosae) dos campos ferruginosos do Parque Estadual do Itacolomi, MG, Brasil: florística e fenologia. Rev. Bras. Bot. 28:493-504.

DUTRA, V.F., GARCIA, F.C.P. \& LIMA, H.C. 2008a Mimosoideae (Leguminosae) nos campos rupestres do Parque Estadual do Itacolomi, Minas Gerais, Brasil. Rodriguésia 59:573-585.

DUTRA, V.F., GARCIA, F.C.P. \& LIMA, H.C. 2008b. Caesalpinioideae (Leguminosae) nos Campos Rupestres do Parque Estadual do Itacolomi, Estado de Minas Gerais, Brasil. Acta Bot. Brasilica 22:543-554.

DUTRA, V.F., GARCIA, F.C.P. \& LIMA, H. 2009. Papilionoideae (Leguminosae) nos Campos Rupestres do Parque Estadual do Itacolomi, MG, Brasil. Acta Bot. Brasil. 23:145-159.

DUTRA, V.F.; LIMA, L.C.P.; GARCIA, F.C.P.; LIMA, H.C. \& SARTORI, A.L.B. 2014. Geographic distribution patterns of Leguminosae and their relevance for the conservation of the Itacolomi State Park, Minas Gerais, Brazil. Biota Neotrop 14(1): e20133937.

ECHTERNACHT, L., TROVÓ, M., OLIVEIRA, C.T. \& PIRANI, J.R. 2011. Areas of endemism in the Espinhaço Range in Minas Gerais, Brazil. Flora 206:782-791.

EITEN, G. 1983. Classificação da vegetação do Brasil. Conselho Nacional de Desenvolvimento Científico e Tecnológico, Brasília

ENVIRONMENTAL SYSTEMS RESEARCH INSTITUTE. 2007. ArcEditor Desktop GIS with advanced geographic data editing and management. http:// www.esri.com/software/arcgis/arceditor/index.html (last access in Jan 24th, 2007).

FERNANDES, G.W., TOMA, T.S.P, ANGRISANO, P. \& OVERBECK, G. Challenges in the Restoration of Quartzitic and Ironstone Rupestrian Grasslands. In Ecology and conservation of mountaintop grasslands in Brazil (G.W. Fernandes, Ed.) Springer, Switzerland, p.449-478

FERREIRA M.T.M. 2011. Composição florística e distribuição vertical de epífitas vasculares sobre indivíduos de Guapira opposita (Vell) Reitz (Nyctaginaceae) em um fragmento florestal na Serra da Brígida, Ouro Preto, MG. Master Dissertation, Federal University of Ouro Preto.

FERRI, M.G. 1980. Vegetação brasileira. EDUSP, São Paulo.

FLORA DO BRASIL 2020 em construção. 2016. Jardim Botânico do Rio de Janeiro. http://floradobrasil.jbrj.gov.br/reflora/listaBrasil/ConsultaPublicaUC/ ConsultaPublicaUC.do\#CondicaoTaxonCP (last access in May 24th, 2016).

FORZZA, R.C., MENINI-NETO L., SALIMENA, F.R.G. \& ZAPPI, D. 2013. Fanerógamas do Parque Estadual do Ibitipoca e suas relações florísticas com outras áreas com campo rupestre de Minas Gerais. In Flora do Parque Estadual de Ibitipoca e seu entorno (R.C. Forzza, L. Menini-Neto, F.R.G. Salimena \& D. Zappi, Orgs.) Editora UFJF, Juiz de Fora, p.153-292.

FUJACO, M.A.G., LEITE, M.G.P. \& MESSIAS, M.C.T.B. 2010. Análise multitemporal das mudanças no uso e ocupação do Parque Estadual do Itacolomi (MG) através de técnicas de geoprocessamento. R. Esc. Min. 63: 695-701.

FUNDAÇÃO SOS MATAATLÂNTICA. 1998. Atlas da evolução dos remanescentes florestais e ecossistemas associados no domínio da Mata Atlântica no Período 1990-1995. Fundação SOS Mata Atlântica, Instituto Nacional de Pesquisas Espaciais / Instituto Socioambiental, São Paulo, Brasil.

GASTAUER, M., MESSIAS, M.C.T.B. \& MEIRA-NETO, J.A.A. 2012. Floristic Composition, Species Richness and Diversity of Campo Rupestre Vegetation from the Itacolomi State Park, Minas Gerais, Brazil. Environment and Natural Resources Research 2:115-128

GIULIETTI, A.M., MENEZES, N.M., PIRANI, J.R., MEGURO, M. \& WANDERLEY, M.G.L. 1987. Flora da Serra do Cipó, Minas Gerais: caracterização e lista das espécies. Bol. Bot. Univ. São Paulo 9:1-51.

GIULIETTI, A.M. \& PIRANI, J.R. 1988. Patterns of geographic distribution of some plant species from the Espinhaço Range, Minas Gerais and Bahia, Brazil. In Workshop on Neotropical Distribution Patterns (W.R. Heyer \& P.E Vanzolini, eds.). Academia Brasileira de Ciências, Rio de Janeiro, p.39-69.

GIULLIETI, A.M., PIRANI, J.R. \& HARLEY, R.M. 1997. Espinhaço Range region, Eastern Brazil. In Centres of plant diversity: a guide and strategy for their conservation. (S.D. Davis, V.H. Heywood, O. Herrera-Macbride, J. Villa-Lobos \& A.C. Hamilton, eds). Information Press, Oxford, p.397-404.

HARLEY, R.M. 1988. Evolution and distribution of Eriope (Labiatae), and its relatives, in Brazil. In Proceedings of a workshop on neotropical distribution patterns (P.E. Vanzolini \& W.R. Heyer, eds). Academia Brasileira de Ciências, Rio de Janeiro, p.71-120.

HARLEY, R.M. 1995. Introduction. In Flora of the Pico das Almas, Chapada Diamantina - Bahia, Brazil. (B.L. Stannard, Y.B. Harvey \& R.M. Harley, eds.). Royal Botanic Gardens, Kew, p.1-42.

HARLEY, R.M. \& SIMMONS, N.A. 1986. Florula of Mucugê, Chapada Diamantina - Bahia, Brazil. Royal Botanic Gardens, Kew.

HUECK, K. 1953. Distribuição e habitat natural do Pinheiro do Paraná (Araucaria angustifolia). Bol. Fac. Filos. Ciênc. Univ. São Paulo, Bot. 10:1-24.

IEF. 2016. Parque Estadual do Itacolomi. http://www.ief.mg.gov.br/parques/itacolomi/ itacolomi.asp (last access in Feb 15th, 2016)

IUCN 2015. The IUCN Red List of Threatened Species. Version 2015-4. http:// www.iucnredlist.org. (last access in Nov 12th, 2015).

JACOBI, C.M., CARMO, F.F., VINCENT, R.C. \& STEHMANN, J.R. 2007. Plant communities on ironstone outcrops: a diverse and endangered Brazilian ecosystem. Biodivers. Conserv. 16:2185-2200.

JBRJ - Instituto de Pesquisas Jardim Botânico do Rio de Janeiro. 2016. Jabot Banco de Dados da Flora Brasileira. http://www.jbrj.gov.br/jabot. (last access in Jun 15th, 2016).

KAMINO, L.H.Y., OLIVEIRA-FILHO, A.T. \& STEHMANN, J.R. 2008. Relações florísticas entre as fitofisionomias florestais da Cadeia do Espinhaço, Brasil. Megadiversidade 4:38-77.

LENZA, E. \& KLINK, C.A. 2006. Comportamento fenológico de espécies lenhosas em um cerrado sentido restrito de Brasília, DF. Rev. Bras. Bot. 29:627-638. 
LIMA, L.C.P., GARCIA, F.C.P. \& SARTORI, A.L.B. 2007. Leguminosae nas florestas estacionais do Parque Estadual do Itacolomi, Minas Gerais, Brasil: ervas, arbustos, subarbustos, lianas e trepadeiras. Rodriguésia 58:331-358.

LIMA, L.C.P., GARCIA, F.C.P. \& SARTORI, A.L.B. 2010. As Leguminosae arbóreas das florestas estacionais do Parque Estadual do Itacolomi, Minas Gerais, Brasil. Rodriguésia 61:441-466.

MESSIAS, M.C.T.B., GARCIA, M.G.P., MEIRA NETO, J.A.A. \& KOZOVITS, A.R. 2011. Life-form spectra of quartzite and itabirite rocky outcrop sites, Minas Gerais, Brazil. Biota Neotrop. 11:1-14.

MESSIAS, M.C.T.B., LEITE, M.G.P., MEIRA-NETO, J.A.A. \& KOZOVITS, A.R. 2012. Fitossociologia de campos rupestres quartzíticos e ferruginosos no Quadrilátero Ferrífero, Minas Gerais. Acta Bot. Bras. 26:230-242.

MESSIAS, M.C.T.B., LEITE, M.G.P., MEIRA-NETO, J.A.A., KOZOVITS, A.R. \& TAVARES, R. 2013. Soil-Vegetation Relationship in Quartzitic and Ferruginous Brazilian Rocky Outcrops Folia Geobot. 48:509-521.

MINISTÉRIO DO MEIO AMBIENTE (MMA). 2014. Portaria No 443, de 17 de dezembro de 2014. Lista nacional oficial de espécies da flora ameaçadas de extinção. http://pesquisa.in.gov.br/imprensa/jsp/visualiza/index.jsp?data=18/12/2 014\&jornal=1\&pagina=110\&totalArquivos=144 (last access in Feb 15th, 2015)

MELLO-BARRETO, H.L. 1942. Regiões fitogeográficas de Minas Gerais. Boletim do Departamento Geográfico de Minas Gerais 4:9-30.

MORELLATO, L.P.C. \& HADDAD, C.F.B. 2000. Introduction: The Brazilian Atlantic Forest. Biotropica 32:786-792.

MYERS, N., MITTERMEIER, R.A., MITTERMEIER, C.G., FONSECA, G.A.B \& KENT, J. 2000. Biodiversity hotspots for conservation priorities. Nature 403:853-858.

OLIVEIRA-FILHO, A.T. \& FLUMINHAN-FILHO, M. 1999. Ecologia da vegetação do Parque Florestal Quedas do Rio Bonito. Cerne 5:50-63.

PEDREIRA, G. \& SOUSA, H.C. 2011. Comunidade arbórea de uma mancha florestal permanentemente alagada e de sua vegetação adjacente em Ouro Preto-MG, Brasil. Cienc. Florest. 21:663-675.

PERON, M.V. 1989. Listagem preliminar da flora fanerogâmica dos Campos Rupestres do Parque Estadual do Itacolomi, Ouro Preto/Mariana, MG Rodriguésia 67:63-69.

PIRANI, J.R., GIULIETTI, A.M., MELLO-SILVA, R. \& MEGURO, M. 1994 Checklist and patterns of geographic distribution of the vegetation of Serra do Ambrósio, Minas Gerais, Brazil. Rev. Bras. Bot.17:133-147.

PIRANI, J.R., MELLO-SILVA, R. \& GIULIETTI, A.M. 2003. Flora de Grão-Mogol, Minas Gerais, Brasil. Bol. Bot. Univ. São Paulo 21:1-24.
REICH, P.B. \& BORCHET, R. 1984. Water stress and tree phenology in a tropical dry forest in the lowlands of Costa Rica. J. Ecol. 72:61-74.

RIBEIRO, M.C., METZGER, J.P., MARTENSEN, A.C., PONZONI, F. \& HIROTA, M.M. 2009. Brazilian Atlantic forest: how much is left and how is the remaining forest distributed? Implications for conservation. Biol. Conserv. 142:1141-1153.

RIZZINI, C.T. 1997. Tratado de fitogeografia do Brasil: aspectos ecológicos, sociológicos e florísticos. Âmbito Cultural, Rio de Janeiro.

SCHAEFER, C.E.G.R., CORRÊA, G.R., CANDIDO, H.G., ARRUDA, D.M., NUNES, J.A., ARAUJO, R.W., RODRIGUES, P.M.S., FERNANDES FILHO, E.I., PEREIRA, A.F.S. BRANDÃO, P.C. \& NERI, A.V. The Physical Environment of Rupestrian Grasslands (Campos Rupestres) in Brazil: Geological, Geomorphological and Pedological Characteristics, and Interplays. In Ecology and Conservation of Mountaintop Grasslands in Brazil (G.W. Wilson, Ed.) Springer, Switzerland, p.15-40.

SCOLFORO, J.R.S., MACHADO, E.L.M., SILVA, C.P.C., MELLO, J.M. \& OLIVEIRA-FILHO, A.T. 2008. Definição de grupos fisionômicos na floresta estacional semidecidual e na floresta ombrófila. In Inventário Florestal de Minas Gerais: Floresta estacional semidecidual e ombrófila - Florística, estrutura, diversidade, similaridade, distribuição diamétrica e de altura, volumetria, tendências de crescimento e áreas aptas para manejo florestal (J.R.S. Scolforo, J.M. Mello \& C.P.C. Silva, eds.). Editora UFLA, Lavras, cap. 1, p.91-192.

STANNARD, B.L. 1995. Flora of the Pico das Almas, Chapada Diamantina, Bahia, Brazil. Kew, Royal Botanic Gardens.

THE PLANT LIST. 2013. Version 1.1. http://www.theplantlist.org (last access in Jan 21st, 2016).

THIERS, B. 2016. [continuously updated]. Index Herbariorum: A global directory of public herbaria and associated staff. New York Botanical Garden's Virtual Herbarium. http://sweetgum.nybg.org/science/ih/ (last access in Oct 10st, 2016).

VALIM, E.A.R., NALINI-JR, H.A. \& KOZOVITS, A.R. 2013. Litterfall dynamics in a iron-rich rock outcrop complex in the southeastern portion of the Iron Quadrangle of Brazil. Acta Bot. Brasilica 27:286-293.

VELOSO, H.P., RANGEL-FILHO, A.L.R. \& LIMA, J.C.A. 1991. A classificação da vegetação brasileira adaptada a um sistema universal. IBGE, Rio de Janeiro.

Received: 27/07/2016

Revised: 04/01/2017

Accepted: 05/01/2017

Published online: 30/01/2017 